\title{
A TEST FOR WEAK STATIONARITY IN THE SPECTRAL DOMAIN
}

\author{
JAVIER HIDALGO AND PEDRO CL SOUZA
}

\begin{abstract}
We examine a test for weak stationarity against alternatives that covers both local-stationarity and break point models. A key feature of the test is that its asymptotic distribution is a functional of the standard Brownian bridge sheet in $[0,1]^{2}$, so that it does not depend on any unknown quantity. The test has non-trivial power against local alternatives converging to the null hypothesis at a $T^{-1 / 2}$ rate, where $T$ is the sample size. We also examine an easy-to-implement bootstrap analogue and present the finite sample performance in a Monte Carlo experiment. Finally, we implement the methodology to assess the stability of inflation dynamics in the United States and on a set of neuroscience tremor data.
\end{abstract}

\section{INTRODUCTION}

Weak stationarity, the property whereby the structure of the data in their first two moments is independent of time, plays an important and key role when invoking asymptotic arguments, making inferences on a time series sequence or making accurate predictions of future values. However, the assumption of weak stationarity is difficult to justify a priori, and it could be possible that some sequences exhibit nonstationary behavior. In economics, a well-known example is Lucas's (1976) critique. The justification is the belief that the parameters of macroeconometric models may depend implicitly on agents' expectations and thus are unlikely to remain stable as policymakers change their behavior. In addition, the possibility of data exhibiting nonstationary behavior is not constrained to economic data sets, see examples in Paparoditis (2009) or Dahlhaus (2009). Thus, the purpose of this paper is to present a test for weak stationarity that is easy to implement. We are not concerned, however, with the situation in which the possible change in the dynamics is due to a random variable as in SETAR, threshold or Markov switching models. These latter models are regarded as nonlinear, and within these types of models, one is often more concerned with testing for linearity.

Testing for weak stationarity is not a new endeavour. There are two main approaches. The first one, focusing on change points, assumes that the practitioner knows the parametric model that generates the data. See, for example, Picard (1985), Davis et al. (1995), and the surveys by Perron (2006) or Aue and Horvath (2013). The second, and more recent, approach describes testing procedures when the practitioner does not have a parametric model in mind or she is not confident about one. See, among others, Paparoditis (2009) or, under Gaussianity, Preuß et al. (2013) in the context of the so-called localstationary models or evolutionary spectra introduced by Priestley (1965). However, their approaches are quite different. Paparoditis (2009), using ideas of Härdle and Mammen (1993) for model specification, employs a direct comparison of two different nonparametric fits of the spectral density function, one under the null hypothesis and a second one under the alternative. By contrast, the approach of Preuß et al. (2013) is based on the empirical spectral distribution function, similar to Dahlhaus and Polonik (2009); see Grenander and Rosenblatt (1957) for earlier ideas.

Date: 14 April 2018.

Key words and phrases. Welch's estimator. Time-varying spectral density. Bootstrap methods.

We thank the Associate Editor and two referees for very helpful comments. Any remaining errors are our sole responsibility. 
In this paper, the primary interest is to present and examine testing procedures for the hypothesis of weak or covariance stationarity when the practitioner does not have a parametric model in mind. The methodology exploits the well-known property that under weak stationarity, the periodograms at two different Fourier frequencies are asymptotically independent, and hence, our approach parallels Dwivedi and Subba Rao (2011) and Jentsch and Subba Rao (2015), who rely on a similar result but for the discrete Fourier transform. Although the implementation of the test by Dwivedi and Subba Rao (2011) differs substantially from ours (see Section 2 for details), the common feature among all of these papers is that the asymptotic distribution does not depend on the second-order dependence of the sequence, contrary to the works mentioned in the previous paragraph.

However, there are some differences between the methodology in the aforementioned papers and ours. First, implementing our test only requires the choice of one bandwidth parameter, which appears to be a minimal requirement, as we do not specify any parametric model even under the null hypothesis. This is in contrast, for instance, to the approaches of Paparoditis (2009) and Dwivedi and Subba Rao (2011), which require the choice of 3 or even 4 different bandwidth parameters for their implementation. Thus, our methodology appears easier to implement, and reduces the possible sensitivity of the bandwidth parameter choice to the size and/or power of the test in small samples. Second, our test detects local alternatives of order $T^{-1 / 2}$, with $T$ being the sample size. Hence, our tests are more efficient than those that only detect local alternatives of order $T^{-\alpha}$ for some $\alpha<1 / 2$, as is the case using Härdle and Mammen's (1993) methodology. On the other hand, it is worth mentioning that Jentsch and Subba Rao (2015) allow for nonlinear structures, whereas our approach assumes that the model has a linear one, see Condition $C 1$ below. Whether our methodology can be extended to nonlinear sequences, under a condition similar to Assumption 3.1 of Jentsch and Subba Rao (2015), is worth exploring but it is beyond the scope of the paper.

A second goal of the paper is to describe and examine a bootstrap analogue of the test. The motivation comes from the fact that our Monte Carlo experiment suggests that the asymptotic distribution seems not to provide a good approximation for the finite sample distribution. One feature of our bootstrap is that it does not require the choice of any additional bandwidth parameter for its implementation, in contrast to the methodology suggested by Dwivedi and Subba Rao (2011) or more recently by Preuß et al. (2013). Our bootstrap algorithm echoes the approaches of Hidalgo (2007) and Hidalgo and Seo (2015) for lattice data exhibiting long memory dependence, which it is well-known not to be strong mixing, see Ibragimov and Rozanov (1978). As a by-product, we present a very simple estimator of the fourth cumulant, although we do not pursue its comparison to that of Grenander and Rosenblatt (1957) or its time domain analogue examined by Fragkeskou and Paparoditis (2016). Such efforts are beyond the scope of this paper.

The remainder of the paper is organized as follows. The next section describes and examines the test for the null hypothesis of covariance stationarity, the asymptotic behavior of which does not depend on any unknown quantity nor, in particular, on the spectral density function. We also discuss an alternative test, the asymptotic distribution of which only depends on the fourth cumulant of the innovations. Section 3 presents a valid bootstrap algorithm for our testing methodology. Section 4 presents a Monte Carlo experiment to shed light on the finite sample performance of the test. The Monte Carlo experiment seems to indicate that our test has better performance (both in terms of size and power) across data generating processes than other comparable tests, such as that suggested by Preuß et al. (2013). We also implement the test with two real data sets. Section 5 is the conclusion, with the proofs given in the Appendix. 


\section{THE TEST AND REGULARITY CONDITIONS}

We are interested in testing the null hypothesis, $H_{0}$, that the observed process $\left\{x_{t}\right\}_{t \in \mathbb{Z}}$ is covariance stationary against the alternative that the covariance of the sequence changes over time. For that purpose, we assume that under the null hypothesis the process $\left\{x_{t}\right\}_{t \in \mathbb{Z}}$ has a $M A(\infty)$ representation. That is,

\section{Condition C1:}

$$
x_{t}=\sum_{j=0}^{\infty} \beta(j) \varepsilon_{t-j}, \quad \beta(0)=1, \quad \sum_{j=0}^{\infty} j|\beta(j)|<\infty,
$$

where $\left\{\varepsilon_{t}\right\}_{t \in \mathbb{Z}}$ is an independent and identically distributed (iid) sequence with $\mathcal{E}\left(\varepsilon_{t}\right)=0$, $\mathcal{E}\left(\varepsilon_{t}^{2}\right)=\sigma_{\varepsilon}^{2}$ and finite 8 th moments. We denote the fourth cumulant of $\varepsilon_{t} / \sigma_{\varepsilon}$ as $\kappa_{4}$. In addition the modulus of

$$
\mathcal{B}(z)=\sum_{j=0}^{\infty} \beta(j) e^{-i j z}
$$

is bounded away from zero for all $z \in[-\pi, \pi]$.

Condition $C 1$ is standard and very mild. It implies that the sequence is weakly or covariance stationary, e.g.

$$
E\left(x_{t} x_{t+\ell}\right)=\gamma(|\ell|) ; \quad \sum_{\ell=0}^{\infty} \ell|\gamma(\ell)|<\infty
$$

That is, $\{\gamma(\ell)\}_{\ell \in \mathbb{Z}}$ is independent of time. Also Condition $C 1$, i.e. (2.1) and (2.3), implies that the sequence $\left\{x_{t}\right\}_{t \in \mathbb{Z}}$ has a spectral density function,

$$
f(\lambda)=\frac{1}{2 \pi} \sum_{\ell=-\infty}^{\infty} \gamma(\ell) e^{i \ell \lambda}=: \frac{\sigma_{\varepsilon}^{2}}{2 \pi}|\mathcal{B}(\lambda)|^{2}, \quad \lambda \in[0, \pi],
$$

which is also independent of time. So we can formulate our null hypothesis, $H_{0}$, as the spectral density function of the sequence $\left\{x_{t}\right\}_{t \in \mathbb{Z}}$ does not vary with time. It is worth noting that using the autoregressive representation $x_{t}-\sum_{j=1}^{\infty} \alpha(j) x_{t-j}=\varepsilon_{t}$ we can write the spectral density function as $f(\lambda)=\sigma_{\varepsilon}^{2}|\mathcal{A}(\lambda)|^{-2} / 2 \pi$, where $\mathcal{B}^{-1}(z)=: \mathcal{A}(z)=1-\sum_{j=1}^{\infty} \alpha(j) e^{-i j z}$.

Finally, it appears that we may relax Condition $C 1$ to allow the conditions stated by Dalla, Giraitis and Hidalgo (2005). For instance, at the expense of complicating the technical apparatus, we can drop the assumption of independence of $\left\{\varepsilon_{t}\right\}_{t \in \mathbb{Z}}$ to allow for martingale difference sequences in its first and second moments, meaning that $C 1$ would become similar to Assumption A1 of Dwivedi and Subba Rao (2011).

We introduce some notation before describing and motivating the test. We take a stretch of data $\left\{x_{t}\right\}_{t=1}^{T}$, where $T$ denotes the sample size, and divide it into $\mathbf{B}$ blocks, each of length $n$, that is, $\mathbf{B}=T / n$. Thus, the $b$-th block is based on the observations $\left\{x_{t+(b-1) n}\right\}_{t=1}^{n}$. Then, denote the periodogram of the $b$-th block of observations $\left\{x_{t+(b-1) n}\right\}_{t=1}^{n}$ by

$$
I_{x, b}(j)=\frac{1}{n}\left|\sum_{t=1}^{n} x_{t+(b-1) n} e^{-i t \lambda_{j}}\right|^{2}, b=1, \ldots, \mathbf{B},
$$

where $\lambda_{j}=2 \pi j / n, j=1, \ldots,[n / 2]=: \tilde{n}$, and where we abbreviate in what follows $g\left(\lambda_{j}\right)$ by $g(j)$ for a generic function $g(\lambda)$. Similarly, the periodogram of $\left\{\varepsilon_{t+(b-1) n}\right\}_{t=1}^{n}, b=1, \ldots, \mathbf{B}$, is given by $I_{\varepsilon, b}(j)=n^{-1}\left|\sum_{t=1}^{n} \varepsilon_{t+(b-1) n} e^{-i t \lambda_{j}}\right|^{2}$. We have the following condition on $\mathbf{B}$ and/or $n$. 
Condition C2: $n$ is such that as $T$ increases to infinity, $\frac{T}{n^{2}}+\frac{n^{3}}{T^{2}} \rightarrow 0$ and $\mathbf{B}=T / n$.

Our definition of the periodogram in (2.4) is similar to that of Dahlhaus (1997), i.e.

$$
I_{x, b}(j)=\frac{1}{n}\left|\sum_{t=-\tilde{n}+1}^{\tilde{n}} x_{t+(b-1) n} e^{-i t \lambda_{j}}\right|^{2},
$$

which has the interpretation of being the periodogram over a segment of length $n$ with its midpoint at $t+(b-1) n$. There is no difference between this and our definition from an asymptotic perspective, and we prefer (2.4) for notational simplicity.

We now describe and motivate the test. Suppose that we were interested in the null hypothesis $H_{0}$, but only for a specific frequency $\lambda_{j}, j=1, \ldots, \tilde{n}$. That is, the spectral density function of $\left\{x_{t}\right\}_{t \in \mathbb{Z}}$ at $\lambda_{j}, f(j)$, does not depend on time. Then, since Bartlett's decomposition, see e.g. Brockwell and Davis (1991), implies that, for all $b=1, \ldots, \mathbf{B}, I_{x, b}(j) / f(j) \simeq I_{\varepsilon, b}(j) / \sigma_{\varepsilon}^{2}$, and $\left\{\left(I_{\varepsilon, b}(j) / \sigma_{\varepsilon}^{2}\right)-1\right\}_{b=1}^{\mathbf{B}}$ behaves as a sequence of uncorrelated centered $\chi_{2}^{2}$ random variables, it suggests then to employ the CUSUM statistic

$$
\mathcal{T}_{n, \mathbf{B}}\left(\frac{\mathbf{b}^{*}}{\mathbf{B}} ; j\right)=\frac{1}{\mathbf{B}} \sum_{b=1}^{\mathbf{b}^{*}}\left\{\frac{I_{x, b}(j)}{\widehat{f}(j)}-1\right\}, \mathbf{b}^{*}=2, \ldots, \mathbf{B},
$$

where

$$
\widehat{f}(j)=\frac{1}{\mathbf{B}} \sum_{v=1}^{\mathbf{B}} I_{x, v}(j)
$$

The estimator of the spectral density function $f(j)$ proposed by Welch $(1967), \widehat{f}(j)$, appears to be a natural estimator in our context.

The previous arguments were given for a particular frequency $\lambda_{j}$. However since for all $b_{1}, b_{2}=1, \ldots, \mathbf{B}$, $\operatorname{Cov}\left(I_{\varepsilon, b_{1}}(j) ; I_{\varepsilon, b_{2}}(k)\right)=0$ if $j \neq k$, extending the previous arguments to $[0, \pi]$, we might suggest a test for $H_{0}$ based on

$$
\mathcal{T}_{n, \mathbf{B}}\left(\frac{\mathbf{b}^{*}}{\mathbf{B}} ; \frac{\mathbf{j}^{*}}{\tilde{n}}\right)=\frac{1}{\tilde{n}} \sum_{j=1}^{\mathbf{j}^{*}} \mathcal{T}_{n, \mathbf{B}}\left(\frac{\mathbf{b}^{*}}{\mathbf{B}} ; j\right), \quad \mathbf{j}^{*}=1, \ldots, \tilde{n}
$$

As noted in the introduction, the statistic (2.6), or (2.7) given below, has some similarities to that given by Dwivedi and Subba Rao (2011); see also Jentsch and Subba Rao (2015), and Bandyopadhy et al. (2017). Specifically, denote $\omega_{k}=2 \pi k / T$ and $\mathcal{J}_{x}\left(\omega_{k}\right)=T^{-1 / 2} \sum_{t=1}^{T} x_{t} e^{-i t \omega_{k}}, k=1, \ldots T$, and compute

$$
\widehat{c}_{T}(r)=\frac{1}{T} \sum_{k=1}^{T} \frac{\mathcal{J}_{x}\left(\omega_{k}\right) \mathcal{J}_{x}\left(\omega_{k+r}\right)}{\widehat{f}^{1 / 2}\left(\omega_{k}\right) \widehat{f}^{1 / 2}\left(\omega_{k+r}\right)}, \quad r=1, \ldots, m,
$$

for some finite chosen $m$, with $\widehat{f}\left(\omega_{k}\right)$ being the weighted average periodogram estimator. Then, using the fact that under $H_{0},\left\{T^{1 / 2} \widehat{c}_{T}(r)\right\}_{r=1}^{[T / 2]}$ behaves as a sequence of independent random variables, they propose a test based on

$$
\mathcal{D S}_{T}(m)=T \sum_{r=1}^{m}\left\{\left|\widehat{c}_{T}(r)\right|^{2} /\left(1+\widehat{\kappa}_{4}\left(\omega_{r}\right)\right)^{1 / 2}\right\},
$$

where $1+\widehat{\kappa}_{4}\left(\omega_{r}\right)$ is an estimator of the second moment of $T\left|\widehat{c}_{T}(r)\right|^{2}$. The difference between $\mathcal{T}_{n, \mathbf{B}}\left(\frac{\mathbf{b}^{*}}{\mathbf{B}} ; \frac{\mathbf{j}^{*}}{\tilde{n}}\right)$ and $\mathcal{D S R}_{T}(m)$ bears some similarities to those that exist between the goodness-of-fit test of Grenander and Rosenblatt (1957) and Portmanteau tests. This is the case after we observe that the Cramér-vonMises statistic based on $\mathcal{T}_{n, \mathbf{B}}\left(\frac{\mathbf{b}^{*}}{\mathbf{B}} ; \frac{\mathbf{j}^{*}}{\tilde{n}}\right)$ is a weighted version of $\mathcal{D} \mathcal{S} \mathcal{R}_{T}(m)$ with $m=[T / 2]$, as the test 
of Grenander and Rosenblatt (1957) is a weighted version of the Portmanteau test with $m=[T / 2]$. See also Delgado, Hidalgo and Velasco's (2005) Section 3 for details.

However, the asymptotic distribution of $\mathcal{T}_{n, \mathbf{B}}\left(\frac{\mathbf{b}^{*}}{\mathbf{B}} ; \frac{\mathbf{j}^{*}}{\tilde{n}}\right)$ depends on $\kappa_{4}$, and hence, it is not pivotal; see Proposition 2 below. We then propose the following statistic:

$$
\mathcal{T} \mathcal{P}_{n, \mathbf{B}}\left(\frac{\mathbf{b}^{*}}{\mathbf{B}} ; \frac{\mathbf{j}^{*}}{\tilde{n}}\right)=\frac{1}{\tilde{n}} \sum_{j=1}^{\mathbf{j}^{*}} \mathcal{T} \mathcal{P}_{n, \mathbf{B}}\left(\frac{\mathbf{b}^{*}}{\mathbf{B}} ; j\right), \mathbf{j}^{*}=1, \ldots, \tilde{n}
$$

as the basis to test for $H_{0}$, where

$$
\mathcal{T P}_{n, \mathbf{B}}\left(\frac{\mathbf{b}^{*}}{\mathbf{B}} ; j\right)=\frac{1}{\mathbf{B}} \sum_{b=1}^{\mathbf{b}^{*}}\left\{\frac{I_{x, b}(j) / \widehat{\sigma}_{\varepsilon}^{2}(b)}{\frac{1}{\mathbf{B}} \sum_{v=1}^{\mathbf{B}}\left(I_{x, v}(j) / \widehat{\sigma}_{\varepsilon}^{2}(v)\right)}-1\right\}, \quad \mathbf{b}^{*}=2, \ldots, \mathbf{B}
$$

and

$$
\widehat{\sigma}_{\varepsilon}^{2}(b)=\frac{1}{n} \sum_{t=1}^{n} \widehat{\varepsilon}_{t+(b-1) n}^{2}, \quad b=1, \ldots, \mathbf{B}
$$

is an estimator of the variance of $\varepsilon_{t}$ in the $b$-th block, and $\widehat{\varepsilon}_{t}$ is given in (2.15) below. The motivation comes after observing, see e.g. Anderson and Walker (1964), that for linear processes, such as those considered in this paper, the asymptotic distribution of the estimator of the correlation coefficient depends only on the first two moments. This is in contrast with the estimator of the covariance that depends on the fourth cumulant. It is worth mentioning that these properties depend crucially on the validity of Bartlett's decomposition which assumes a linear process.

Because the implementation of $\mathcal{T} \mathcal{P}_{n, \mathbf{B}}\left(\frac{\mathbf{b}^{*}}{\mathbf{B}} ; \frac{\mathbf{j}^{*}}{\tilde{n}}\right)$ in (2.7), as is the case with our estimator of $\kappa_{4}$ and the bootstrap, requires obtaining $\left\{\varepsilon_{t}\right\}_{t \in \mathbb{Z}}$, we first provide a simple method to obtain the innovations $\varepsilon_{t}$. For this purpose, given a generic sequence $\left\{z_{t}\right\}_{t=1}^{T}$, we denote the discrete Fourier transform $(D F T)$ of $\left\{z_{t+(b-1) n}\right\}_{t=1}^{n}$ by

$$
\mathcal{J}_{z, b}(j)=\frac{1}{n^{1 / 2}} \sum_{t=1}^{n} z_{t+(b-1) n} e^{-i t \lambda_{j}}, \quad j=1, \ldots, \tilde{n}, \quad b=1, \ldots, \mathbf{B} .
$$

It is well known, see expression (10.3.12) of Brockwell and Davis (1991), that under $H_{0}$ and Condition $C 1$, the DFTs of $\left\{\varepsilon_{t+(b-1) n}\right\}_{t=1}^{n}$ and $\left\{x_{t+(b-1) n}\right\}_{t=1}^{n}$ satisfy the following relationship

$$
\mathcal{J}_{x, b}(j)=\mathcal{B}(-j) \mathcal{J}_{\varepsilon, b}(j)+\mathrm{Y}_{n, b}(j ; 0), \quad b=1, \ldots, \mathbf{B},
$$

where $\mathcal{B}(j)=: \mathcal{B}\left(\lambda_{j}\right)$ given in $(2.2)$ and

$$
\mathrm{Y}_{n, b}(j ; a)=\sum_{\ell=a}^{\infty} \beta(\ell) e^{-i \ell \lambda_{j}}\left(\frac{1}{n^{1 / 2}}\left\{\sum_{t=1-\ell}^{n-\ell}-\sum_{t=1}^{n}\right\} \varepsilon_{t+(b-1) n} e^{-i t \lambda_{j}}\right) .
$$

Next, using the inverse transformation of the DFT,

$$
z_{t+(b-1) n}=\frac{1}{n^{1 / 2}} \sum_{j=1}^{n} \mathcal{J}_{z, b}(j) e^{i t \lambda_{j}}, \quad t=1, \ldots, n,
$$


and noting that $\mathrm{Y}_{n, b}(j ; a)$ is negligible compared to $\mathcal{B}(-j) \mathcal{J}_{\varepsilon, b}(j)$ in $(2.10)$, Condition $C 1$ and $H_{0}$ imply that

$$
\begin{aligned}
\varepsilon_{t+(b-1) n} & \simeq \frac{1}{n^{1 / 2}} \sum_{j=1}^{n} e^{i t \lambda_{j}} \mathcal{A}(-j) \mathcal{J}_{x, b}(j) \\
& =\frac{1}{n^{1 / 2}} \sum_{j=1}^{n} e^{i t \lambda_{j}} \mathcal{B}^{-1}(-j) \mathcal{J}_{x, b}(j), \quad t=1, \ldots, n ; b=1, \ldots, \mathbf{B},
\end{aligned}
$$

where " $\approx "$ should be read as "approximately." Recall our definition of $\mathcal{A}(j)$ after expression $(2.2)$.

Thus, to compute $\left\{\widehat{\varepsilon}_{t+(b-1) n}\right\}_{t=1}^{n}$, it suffices to provide an estimator of $\mathcal{A}(j)$. To that end, we employ the canonical spectral decomposition of $f(\lambda)$; see Brillinger (1981, p. $78-79)$ or Hannan (1970). Indeed, observing that $f(\lambda)=\sigma_{\varepsilon}^{2}|\mathcal{A}(\lambda)|^{-2}$, where $\mathcal{A}(\lambda)=\exp \left\{-\sum_{r=1}^{\infty} c_{r} e^{i r \lambda}\right\}$ with

$$
c_{r}=\frac{1}{\pi} \int_{0}^{\pi} \log f(\lambda) \cos (r \lambda) d \lambda, \quad r=0,1, \ldots,
$$

we then estimate $\mathcal{A}(j)$ by

$$
\begin{aligned}
\widehat{\mathcal{A}}(j) & =\exp \left\{-\sum_{r=1}^{[\tilde{n} / 2]} \widehat{c}_{r} e^{i r \lambda_{j}}\right\}, & j=1, \ldots, \tilde{n} \\
\widehat{\mathcal{A}}(j) & =\widehat{\widehat{\mathcal{A}}}(n-j), & j=\tilde{n}+1, \ldots, n-1 \\
\widehat{c}_{r} & =\frac{1}{\tilde{n}} \sum_{\ell=1}^{\tilde{n}} \log \widehat{f}(\ell) \cos r \lambda_{\ell}, & r=0, \ldots, \tilde{n},
\end{aligned}
$$

where $\widehat{f}(\ell)$ is the estimator given in $(2.5)$. Note that $\widehat{\sigma}_{\varepsilon}^{2}=\exp \left(\widehat{c}_{0}\right)$. From here, we compute $\left\{\widehat{\varepsilon}_{t+(b-1) n}\right\}_{t=1}^{n}$ as

$$
\widehat{\varepsilon}_{t+(b-1) n}=\frac{1}{n^{1 / 2}} \sum_{j=1}^{n} e^{i t \lambda_{j}} \widehat{\mathcal{A}}(-j) \mathcal{J}_{x, b}(j), \quad b=1, \ldots, \mathbf{B} .
$$

We finish the section by providing an estimator of $\kappa_{4}$. Given $\left\{\widehat{\varepsilon}_{t}\right\}_{t=1}^{T}$ in $(2.15)$, we compute our estimator of $\kappa_{4}$ as

$$
\widehat{\kappa}_{4}=\frac{1}{T} \sum_{t=1}^{T}\left(\frac{\widehat{\varepsilon}_{t}^{4}}{\widehat{\sigma}_{\varepsilon}^{4}}-3\right),
$$

where either $\widehat{\sigma}_{\varepsilon}^{2}=\exp \left(\widehat{c}_{0}\right)$ or $\widehat{\sigma}_{\varepsilon}^{2}=\mathbf{B}^{-1} \sum_{b=1}^{\mathbf{B}} \widehat{\sigma}_{\varepsilon}^{2}(b)$, with $\widehat{\sigma}_{\varepsilon}^{2}(b)$ defined in (2.8). Notice the latter estimator yields $\widehat{\sigma}_{\varepsilon}^{2}=T^{-1} \sum_{t=1}^{T} \widehat{\varepsilon}_{t}^{2}$.

A major difference between our estimator $\widehat{\kappa}_{4}$ and the rival estimators proposed by Grenander and Rosenblatt (1957) or, more recently, by Paparoditis (2009) and its time domain analogue by Fragkeskou and Paparoditis (2016) is that the latter estimators require for their implementation the choice of at least 2 extra bandwidth parameters, as they depend on the correlation structure of the sequences $\left\{x_{t+(b-1) n}^{2}\right\}_{t=1}^{n}$ and $\left\{x_{t+(b-1) n}\right\}_{t=1}^{n}$. However, ours does not require the choice of any extra bandwidth parameter in its computation.

Proposition 1. Under $H_{0}$ and assuming $C 1$ and $C 2$, we have that $\widehat{\kappa}_{4} \rightarrow_{P} \kappa_{4}$.

Proof. The proof of this proposition, and any other result, is given in the Appendix.

\subsection{Asymptotic properties of (2.7).}


We begin by introducing some regularity conditions in a general format which will encompass the assumptions of $x_{t}$ under the null hypothesis but also the type of models for which the test based on the statistic (2.7) has non-trivial power. In particular we consider, under the alternative hypothesis, the class of locally stationary processes which is a class of processes that can be approximated at a local level by a stationary time series. These processes are introduced by Dahlhaus (1996) where we observe $\left\{x_{t, T}\right\}_{t=1}^{T}$ in an increasing finer grid. Following Dahlhaus and Polonik (2006), $\left\{x_{t, T}\right\}_{t=1}^{T}$ is said to have a time-varying $M A(\infty)$ representation if it satisfies the following condition:

Condition C1': $\left\{x_{t, T}\right\}_{t=1}^{T}, T \in \mathbb{N}^{+}$, is a sequence of random variables defined as

$$
x_{t, T}=\sum_{j=0}^{\infty} \beta_{t, T}(j) \varepsilon_{t-j}, \text { with } \beta_{t, T}(0)=1,
$$

such that $\sup _{t}\left|\beta_{t, T}(j)\right|<v(j), \sum_{j=0}^{\infty} j v(j)<\infty$ and where $\left\{\varepsilon_{t}\right\}_{t \in \mathbb{Z}}$ satisfies the same conditions as in $C 1$. In addition,

$$
\mathcal{B}_{t, T}(\lambda)=\overline{\mathcal{B}_{t, T}(-\lambda)}=\sum_{j=0}^{\infty} \beta_{t, T}(j) e^{-i j \lambda}
$$

with $\bar{a}$ denoting the conjugate of the complex number $a$, satisfies that $\left|\mathcal{B}_{t, T}(\lambda)\right|$ is bounded away from zero for all $\lambda \in[0, \pi]$.

Condition $C 1^{\prime}$ implies that the sequence is weakly dependent, although not necessarily stationary, because $\beta_{t, T}(j)$ may depend on $t$. Model (2.17) also allows for breaks in some (or all) of the coefficients $\beta_{t, T}(j)$, say $\beta_{t, T}(j)=\delta(j) \mathcal{I}\left(t<t_{0}\right)+\beta(j)$ with $\delta(j) \neq 0$ where $\mathcal{I}(\cdot)$ denotes the indicator function. Observe that the null hypothesis means that the coefficients $\beta_{t, T}(j)$ in model $(2.17)$ become just $\beta(j)$, that is

$$
\beta_{t, T}(j)=\beta(j) \text { for all } j \in \mathbb{N}^{+} \text {and } t=1, \ldots, T, T \in \mathbb{N}^{+} .
$$

Next, following Dahlhaus (1997), we have that (2.17) has the (time-varying) spectral representation

$$
x_{t, T}=\frac{1}{(2 \pi)^{1 / 2}} \int_{-\pi}^{\pi} \mathcal{B}_{t, T}(\lambda) \exp (i \lambda t) d \xi(\lambda),
$$

where $\mathcal{B}_{t, T}(\lambda)$ is given in $(2.18)$.

Under Condition $C 2^{\prime}$ below, we can approximate $(2.18)$ by

$$
\mathcal{B}(u ; \lambda)=\sum_{j=0}^{\infty} \beta(u ; j) e^{-i t \lambda}, u \in[0,1] ; \lambda \in[0, \pi],
$$

in the sense that

$$
\sup _{\lambda \in[0, \pi], 1 \leq t \leq T}\left|\mathcal{B}_{t, T}(\lambda)-\mathcal{B}\left(\frac{t}{T} ; \lambda\right)\right|=O\left(\frac{1}{T}\right),
$$

and thus following Dahlhaus (1997), we can define the "time-varying" spectral density function of $\left\{x_{t, T}\right\}_{t=1}^{T}, T \in \mathbb{N}^{+}$, by

$$
f(u ; \lambda)=\frac{\sigma_{\varepsilon}^{2}}{2 \pi}|\mathcal{B}(u ; \lambda)|^{2} \quad u \in[0,1] ; \quad \lambda \in[0, \pi] .
$$

For instance, for the $t v A R M A$ model

$$
x_{t, T}-\sum_{p=1}^{P} \alpha\left(\frac{t}{T} ; p\right) x_{t-p, T}=\sum_{q=0}^{Q} \beta\left(\frac{t}{T} ; q\right) \varepsilon_{t-q},
$$


we have that

$$
\mathcal{B}(u ; \lambda)=\frac{\sum_{q=0}^{Q} \beta(u ; q) \exp (-i \lambda q)}{1-\sum_{p=1}^{P} \alpha(u ; p) \exp (-i \lambda p)},
$$

whereas for the $A R(1)$ model, $x_{t, T}=\alpha(t) x_{t-1, T}+\varepsilon_{t}$, where

$$
\alpha(t)=\alpha_{1} \mathcal{I}\left(t<t_{0}\right)+\alpha_{2} \mathcal{I}\left(t \geq t_{0}\right) ; \quad \alpha_{1} \neq \alpha_{2} ; \quad\left|\alpha_{1}\right|,\left|\alpha_{2}\right|<1,
$$

we have that $\mathcal{B}(u ; \lambda)=:\left(1-\alpha_{1} \exp (-i \lambda)\right)^{-1}$ when $u=t / T<u_{0}=t_{0} / T$ and $=:\left(1-\alpha_{2} \exp (-i \lambda)\right)^{-1}$ when $u \geq u_{0}$. Note that Condition $C 1^{\prime}$ implies that the sequence $\left\{x_{t, T}\right\}_{t=1}^{T}$ admits an autoregressive representation as Dahlhaus (1996) demonstrates. In fact, if we replace (2.17) with

$$
x_{t, T}=\sum_{j=0}^{\infty} \beta\left(\frac{t}{T} ; j\right) \varepsilon_{t-j} ; \quad \beta\left(\frac{t}{T} ; 0\right)=1,
$$

the $t v A R$ model

$$
x_{t, T}=\sum_{p=1}^{P} \alpha\left(\frac{t}{T} ; p\right) x_{t-p, T}+\varepsilon_{t},
$$

under standard regularity conditions on $\{\alpha(u ; j)\}_{j \geq 0}$ for all $u \in[0,1]$, does not have a representation as in (2.23). Under $H_{0}$, the latter displayed model collapses to the standard $A R(P)$ model.

Recalling that alternatively in the spectral domain, we denote the null hypothesis as that the spectral density function of the process is not time varying, then expression (2.22) suggests that we may write the hypothesis testing as

$$
H_{0}: \quad f(u ; \lambda)=f(\lambda) \text { for all } u \in[0,1],
$$

where $f(\lambda)$ corresponds to the spectral density function of the process $x_{t}$ given in Condition $C 1$ and where the alternative hypothesis, $H_{a}$, is the negation of the null, e.g. denoting by $\mu(\cdot)$ the Lebesgue measure,

$$
H_{a}: \mu(\mathcal{U}, \Lambda)>0,
$$

where $(\mathcal{U}, \Lambda)=\{u \in[0,1] ; \lambda \in[0, \pi]: f(u ; \lambda) \neq f(\lambda)\}$. At the end of Section 2.2, we demonstrate that the test based on $\mathcal{T P}_{n, \mathbf{B}}\left(\frac{\mathbf{b}^{*}}{\mathbf{B}} ; \frac{\mathbf{j}^{*}}{\tilde{n}}\right)$ is also consistent against heteroskedastic alternatives, for instance $x_{t, T}=\sigma_{t, T} \varepsilon_{t}$ with $\sigma_{t, T}=\sigma(t / T)$.

Condition C2': $\beta_{t, T}(j)$ satisfies that

$$
\sup _{1 \leq t \leq T}\left|\beta_{t, T}(j)-\beta\left(\frac{t}{T} ; j\right)\right| \leq \frac{C}{T} v(j) \quad \sum_{j=0}^{\infty} j v(j)<\infty .
$$

Condition $C 2^{\prime}$ indicates that $\beta_{t, T}(j)$ can be well approximated (locally) by a smooth function $\beta(u ; j)$ and that observations that are close in time are regarded as stationary. The bound sequence $v(j)$ does not need to be the same as that in Condition $C 1^{\prime}$. However, we keep it for notational simplicity, as both satisfy the upper bound $v(j)=O\left(j^{-2-\delta}\right)$ for some $\delta>0$. Another implication of Condition $C 2^{\prime}$ is that $\mathcal{B}_{t, T}(\lambda)$ and $\mathcal{B}(u ; \lambda)$ given in $(2.18)-(2.20)$ satisfy $(2.21)$. Under $H_{0},(2.26)$ holds trivially.

Note that under Condition $C 1^{\prime},(2.10)$ becomes

$$
\mathcal{J}_{x, b}(j)=\mathcal{B}\left(\frac{n(b-1)}{T} ;-j\right) \mathcal{J}_{\varepsilon, b}(j)+\check{\mathrm{Y}}_{n, b}(j ; 0)+\ddot{\mathrm{Y}}_{n, b}(j),
$$


where $\mathcal{B}\left(\frac{n(b-1)}{T} ; j\right)=: \mathcal{B}\left(\frac{n(b-1)}{T} ; e^{i \lambda_{j}}\right)$ was given in $(2.20)$ and

$$
\begin{aligned}
\check{\mathrm{Y}}_{n, b+1}(j ; a)= & \sum_{\ell=a}^{\infty} \beta\left(\frac{n b}{T} ; \ell\right) e^{-i \ell \lambda_{j}}\left(\frac{1}{n^{1 / 2}}\left\{\sum_{t=1-\ell}^{n-\ell}-\sum_{t=1}^{n}\right\} \varepsilon_{t+b n} e^{-i t \lambda_{j}}\right), \\
\ddot{\mathrm{Y}}_{n, b+1}(j)= & \frac{1}{n^{1 / 2}} \sum_{t=1}^{n}\left\{\left(\sum_{\ell=0}^{\infty}\left(\beta\left(\frac{t+n b}{T} ; \ell\right)-\beta\left(\frac{n b}{T} ; \ell\right)\right) \varepsilon_{t+b n-\ell}\right) e^{i t \lambda_{j}}\right. \\
& \left.+\left(\sum_{\ell=0}^{\infty}\left(\beta_{t+b n, T}(\ell)-\beta\left(\frac{t+n b}{T} ; \ell\right)\right) \varepsilon_{t+b n-\ell}\right) e^{i t \lambda_{j}}\right\} .
\end{aligned}
$$

For reasons that will become clear, it is convenient and useful to examine first the behavior of

$$
\breve{\mathcal{T}}_{n, \mathbf{B}}\left(\frac{\mathbf{b}^{*}}{\mathbf{B}} ; \frac{\mathbf{j}^{*}}{\tilde{n}}\right)=\frac{1}{\tilde{n}} \sum_{j=1}^{\mathbf{j}^{*}} \frac{1}{\mathbf{B}} \sum_{b=1}^{\mathbf{b}^{*}}\left\{\frac{I_{x, b}(j) /\left|\mathcal{B}\left(\frac{n b}{T} ; j\right)\right|^{2}}{\mathbf{B}^{-1} \sum_{v=1}^{\mathbf{B}}\left\{I_{x, v}(j) /\left|\mathcal{B}\left(\frac{n v}{T} ; j\right)\right|^{2}\right\}}-1\right\}
$$

for $\mathbf{b}^{*}=2, \ldots, \mathbf{B}$ and $\mathbf{j}^{*}=1, \ldots, \tilde{n}$. Observe that under $H_{0}, \breve{\mathcal{T}}_{n, \mathbf{B}}\left(\frac{\mathbf{b}^{*}}{\mathbf{B}} ; \frac{\mathbf{j}^{*}}{\tilde{n}}\right)=: \mathcal{T}_{n, \mathbf{B}}\left(\frac{\mathbf{b}^{*}}{\mathbf{B}} ; \frac{\mathbf{j}^{*}}{\tilde{n}}\right)$ as $\left|\mathcal{B}\left(\frac{n b}{T} ; j\right)\right|^{2}$ becomes $|\mathcal{B}(j)|^{2}$. We have the following result.

Proposition 2. Assuming $C 1^{\prime}, C 2^{\prime}$ and $C 2$, we have that as $T \rightarrow \infty$,

$$
[T / 2]^{1 / 2} \breve{\mathcal{T}}_{n, \mathbf{B}}\left(\frac{\mathbf{b}^{*}}{\mathbf{B}} ; \frac{\mathbf{j}^{*}}{\tilde{n}}\right) \stackrel{\text { weakly }}{\Rightarrow} \mathcal{B S}\left([0,1]^{2} ; \kappa_{4}\right)
$$

where $\mathcal{B S}\left([0,1]^{2} ; \kappa_{4}\right)$ is a Gaussian process in $[0,1]^{2}$ with covariance structure

$$
\mathcal{C}\left(\omega_{1}^{*}, \omega_{2}^{*} ; v_{1}^{*}, v_{2}^{*}\right)=\omega_{1}^{*}\left(1-\omega_{2}^{*}\right)\left[v_{1}^{*}+\frac{1}{2} v_{1}^{*} v_{2}^{*} \kappa_{4}\right],\left\{\begin{array}{l}
0 \leq \omega_{1}^{*} \leq \omega_{2}^{*} \leq 1 \\
0 \leq v_{1}^{*} \leq v_{2}^{*} \leq 1
\end{array}\right.
$$

We have the following corollary.

Corollary 1. Let $\varphi(\cdot, \cdot)$ be a continuous functional in $[0,1]^{2} \rightarrow \mathbb{R}^{+}$. Then, under $H_{0}$ and assuming Conditions $C 1$ and $C 2$, we have that

$$
\varphi\left([T / 2]^{1 / 2} \mathcal{T}_{n, \mathbf{B}}\left(\frac{\mathbf{b}^{*}}{\mathbf{B}} ; \frac{\mathbf{j}^{*}}{\tilde{n}}\right)\right) \stackrel{\text { distribution }}{\Rightarrow} \varphi\left(\mathcal{B S}\left([0,1]^{2} ; \kappa_{4}\right)\right) .
$$

Proof. The proof is an immediate consequence of Proposition 2 and the continuous mapping theorem, and thus, is omitted.

The first conclusion that we draw from Proposition 2 is that under Gaussianity, we have that $\mathcal{C}\left(\omega_{1}^{*}, \omega_{2}^{*} ; v_{1}^{*}, v_{2}^{*}\right)=$ $\omega_{1}^{*}\left(1-\omega_{2}^{*}\right) v_{1}^{*}$, which can be regarded as the covariance structure of the "product" of a standard Brownian bridge and a Brownian motion, and hence, $[T / 2]^{1 / 2} \mathcal{T}_{n, \mathbf{B}}\left(\frac{\mathbf{b}^{*}}{\mathbf{B}} ; \frac{\mathbf{j}^{*}}{\tilde{n}}\right)$ is pivotal. This is a major difference with the test proposed by Dette et al. (2011), the covariance structure of which depends on $f(\lambda)$.

However, as the Gaussianity assumption is difficult to justify with many data sets, one route to implement the test would be to employ $\widehat{\kappa}_{4}$, and from there compute the (asymptotic) critical values. This could be achieved by simulating the critical values of $\varphi\left(\mathcal{B S}\left([0,1]^{2} ; \kappa_{4}\right)\right)$ for a mesh $\mathcal{M}$ of possible values of $\kappa_{4}$, denoted by $\operatorname{cr}\left(\kappa_{4}\right)$. Then, given a particular data set, we use as critical values $\operatorname{cr}\left(\widehat{\kappa}_{4}\right)$. A second route is to see whether the statistic $\mathcal{T} \mathcal{P}_{n, \mathbf{B}}\left(\frac{\mathbf{b}^{*}}{\mathbf{B}} ; \frac{\mathbf{j}^{*}}{\tilde{n}}\right)$ in $(2.7)$ depends on $\kappa_{4}$. A third route is to compute valid asymptotic critical values via bootstrap algorithms. The next theorem shows that, indeed, the asymptotic distribution of $\mathcal{T} \mathcal{P}_{n, \mathbf{B}}\left(\frac{\mathbf{b}^{*}}{\mathbf{B}} ; \frac{\mathbf{j}^{*}}{\tilde{n}}\right)$ does not depend on $\kappa_{4}$. 
Theorem 1. Let $\varphi(\cdot, \cdot)$ be a continuous functional in $[0,1]^{2} \rightarrow \mathbb{R}^{+}$. Then, under $H_{0}$ and assuming Conditions $C 1$ and $C 2$, we have that

$$
\begin{aligned}
& \text { (a) }[T / 2]^{1 / 2} \mathcal{T} \mathcal{P}_{n, \mathbf{B}}\left(\frac{\mathbf{b}^{*}}{\mathbf{B}} ; \frac{\mathbf{j}^{*}}{\tilde{n}}\right) \stackrel{\text { weakly }}{\Rightarrow} \mathcal{W B}\left([0,1]^{2}\right), \\
& \text { (b) } \varphi\left([T / 2]^{1 / 2} \mathcal{T} \mathcal{P}_{n, \mathbf{B}}\left(\frac{\mathbf{b}^{*}}{\mathbf{B}} ; \frac{\mathbf{j}^{*}}{\tilde{n}}\right)\right) \stackrel{d}{\Rightarrow} \varphi\left(\mathcal{W B}\left([0,1]^{2}\right)\right)
\end{aligned}
$$

where $\mathcal{W B}\left([0,1]^{2}\right)$ is a Brownian bridge sheet in $[0,1]^{2}$ with covariance structure

$$
\mathcal{C}\left(\omega_{1}^{*}, \omega_{2}^{*} ; v_{1}^{*}, v_{2}^{*}\right)=\omega_{1}^{*}\left(1-\omega_{2}^{*}\right) v_{1}^{*}\left(1-v_{2}^{*}\right) \text {. }
$$

Standard functionals $\varphi(\cdot, \cdot)$ are the Kolmogorov-Smirnov and the Cramér-von-Mises given, respectively, as

$$
\begin{gathered}
\mathcal{K} \mathcal{S}_{n, \mathbf{B}}=\max _{\mathbf{j}^{*}=1, \ldots, \tilde{n} ; \mathbf{b}^{*}=1, \ldots \mathbf{B}}\left|[T / 2]^{1 / 2} \mathcal{T} \mathcal{P}_{n, \mathbf{B}}\left(\frac{\mathbf{b}^{*}}{\mathbf{B}} ; \frac{\mathbf{j}^{*}}{\tilde{n}}\right)\right| \\
\mathcal{C} v \mathcal{M}_{n, \mathbf{B}}=\frac{1}{[T / 2]} \sum_{\mathbf{j}^{*}=1}^{\tilde{n}} \sum_{\mathbf{b}^{*}=1}^{\mathbf{B}}\left|[T / 2]^{1 / 2} \mathcal{T} \mathcal{P}_{n, \mathbf{B}}\left(\frac{\mathbf{b}^{*}}{\mathbf{B}} ; \frac{\mathbf{j}^{*}}{\tilde{n}}\right)\right|^{2} .
\end{gathered}
$$

\subsection{Local Alternatives and Consistency.}

We finish by describing the local alternatives for which the test has non-trivial power. For illustration purposes, we show that $\mathcal{T}_{n, \mathbf{B}}\left(\frac{\mathbf{b}^{*}}{\mathbf{B}} ; \frac{\mathbf{j}^{*}}{\tilde{n}}\right)$ will have a mean different from zero under the alternative hypothesis. Indeed, under Conditions $C 1^{\prime}, C 2^{\prime}$, and $C 2$, we have that

$$
\widehat{f}(j)=: \frac{1}{\mathbf{B}} \sum_{b=1}^{\mathbf{B}} I_{x, b}(j) \stackrel{P}{\rightarrow} \lim _{\mathbf{B} \rightarrow \infty} \frac{1}{\mathbf{B}} \sum_{b=1}^{\mathbf{B}} f\left(\frac{b}{\mathbf{B}} ; j\right) \simeq \int_{0}^{1} f(u ; \lambda(j)) d u,
$$

where $\lambda(j)=\lim _{n(T) \rightarrow \infty} \lambda_{j}$. Thus, as the set $(\mathcal{U}, \Lambda)$ given after expression (2.25) has positive Lebesgue measure, the last displayed expression suggests that

$$
\begin{aligned}
\mathcal{T}_{n, \mathbf{B}}\left(\frac{\mathbf{b}^{*}}{\mathbf{B}} ; \frac{\mathbf{j}^{*}}{\tilde{n}}\right) \simeq & \frac{1}{\tilde{n}} \sum_{j=1}^{\mathbf{j}^{*}} \frac{1}{\mathbf{B}} \sum_{b=1}^{\mathbf{b}^{*}}\left\{\frac{\dot{I}_{x, b}(j)}{\left.\frac{1}{\mathbf{B} \sum_{v=1}^{\mathbf{B}} \dot{I}_{x, v}(j)}-1\right\}}\right. \\
& +\frac{1}{\tilde{n}} \sum_{j=1}^{\mathbf{j}^{*}} \frac{1}{\mathbf{B}} \sum_{b=1}^{\mathbf{b}^{*}}\left\{\frac{f\left(\frac{b}{\mathbf{B}} ; j\right)}{\int_{0}^{1} f(u ; \lambda(j)) d u}-1\right\}\left(1+o_{p}(1)\right),
\end{aligned}
$$

where $\dot{I}_{x, b}(j)=I_{x, b}(j)-f\left(\frac{b}{\mathbf{B}} ; j\right)$. The first term on the right of the last displayed expression is $o_{p}(1)$, proceeding similarly as with the proof of Proposition 2, whereas the second term on the right develops a "mean" different from zero since $f\left(\frac{b}{\mathbf{B}} ; j\right) / \int_{0}^{1} f(u ; \lambda(j)) d u \neq 1$.

Specifically, consider the local alternatives

$$
H_{l}: \quad f(u ; \lambda)=f(\lambda)\left(1+\frac{1}{[T / 2]^{1 / 2}} g(u ; \lambda)\right),
$$

where $g\left(\frac{t}{T} ; \lambda\right)$ is different than zero in the set $(\mathcal{U}, \Lambda)$. It is worth observing that when $H_{l}$ corresponds to an abrupt change at some point in time, $t_{0}$, we have that $g\left(\frac{t}{T} ; \lambda\right)=g(\lambda)$ if $t>t_{0}$, and we could allow $C / T^{1 / 2}<t_{0} / T<1-C / T^{1 / 2}$ for some finite positive constant $C$. Let us introduce the function $d(\cdot ; \cdot)$, defined as

$$
d\left(\omega^{*} ; \pi v\right)=\int_{0}^{\omega^{*}} g(v ; v) d v-\omega^{*} \int_{0}^{1} g(v ; v) d v, \quad \omega^{*} \in[0,1] ; \quad v \in[0,1] .
$$


It is obvious that $d\left(\omega^{*} ; \pi v\right)$ is different from zero unless $f\left(\omega^{*} ; v\right)=: f(v)$ a.e. in $v \in[0, \pi]$ and $\omega^{*} \in[0,1]$. That is, given $v, g\left(\omega^{*} ; \pi v\right)=0$ for all $\omega^{*} \in[0,1]$ if $f\left(\omega^{*} ; v\right)=: f(v)$, i.e. it is constant in the argument $\omega^{*} \in[0,1]$.

Proposition 3. Under $H_{l}$ and assuming $C 1^{\prime}, C 2^{\prime}$, and $C 2$, we have that

(a) $\quad[T / 2]^{1 / 2} \mathcal{T}_{n, \mathbf{B}}\left(\frac{\mathbf{b}^{*}}{\mathbf{B}} ; \frac{\mathbf{j}^{*}}{\tilde{n}}\right) \stackrel{\text { weakly }}{\Rightarrow} D\left(\omega^{*} ; v^{*}\right)+\mathcal{B S}\left([0,1]^{2} ; \kappa_{4}\right)$,

(b) $\quad[T / 2]^{1 / 2} \mathcal{T} \mathcal{P}_{n, \mathbf{B}}\left(\frac{\mathbf{b}^{*}}{\mathbf{B}} ; \frac{\mathbf{j}^{*}}{\tilde{n}}\right) \stackrel{\text { weakly }}{\Rightarrow} \frac{1}{\sigma_{\varepsilon}^{2}} D\left(\omega^{*} ; v^{*}\right)+\mathcal{W B}\left([0,1]^{2}\right)$,

where $v^{*}=\lim _{n=n(T) \rightarrow \infty} \frac{\mathbf{j}^{*}}{\tilde{n}}$ and $\omega^{*}=\lim _{\mathbf{B}=\mathbf{B}(T) \rightarrow \infty} \frac{\mathbf{b}^{*}}{\mathbf{B}}, \mathbf{j}^{*}=1, \ldots, \tilde{n} ; \mathbf{b}^{*}=1, \ldots, \mathbf{B}$ and $D\left(\omega^{*} ; v^{*}\right)=$ $\int_{0}^{v^{*}} d\left(\omega^{*} ; \pi v\right) d v$.

The conclusion from Proposition 3 is that the test has power comparable to its parametric counterparts. The consistency of the tests is standard as the "drift" function $D\left(\omega^{*} ; v^{*}\right)$ is different than zero in a set with positive Lebesgue measure. Thus, our tests have non-trivial power when the alternative converges to the null at the rate $T^{1 / 2}$, which is faster than the $T^{1 / 4}$ obtained elsewhere by Dette et al. (2011) or when using nonparametric fits, although it is the same rate as that of Preuß et al. (2013). However, the latter depends on the assumption that the sequence $\left\{\varepsilon_{t}\right\}_{t \in \mathbb{Z}}$ is Gaussian.

We conclude this section by demonstrating that our tests are able to detect departures from weak stationarity due to heteroskedasticity. Indeed, for illustration purposes, we consider the example given by Dwivedi and Subba Rao (2011), that is, $x_{t, T}=\sigma_{t, T} \varepsilon_{t}$, where $\sigma_{t, T}=\sigma(t / T)$. In this case, standard algebra implies that $E I_{x, b}(j)=\frac{1}{n} \sum_{t=1}^{n} \sigma^{2}((t+b n) / T)$ and

$$
\begin{aligned}
& \frac{1}{\mathbf{B}} \sum_{b=1}^{\mathbf{B}} I_{x, b}(j) \stackrel{P}{\rightarrow} \lim _{T \rightarrow \infty} \frac{1}{\mathbf{B}} \sum_{b=1}^{\mathbf{B}} \frac{1}{n} \sum_{t=1}^{n} \sigma^{2}((t+b n) / T) \\
= & \lim _{T \rightarrow \infty} \frac{1}{\mathbf{B}} \sum_{b=1}^{\mathbf{B}} \sigma^{2}(b / \mathbf{B})\left(1+O\left(\mathbf{B}^{-1}\right)\right)=\int_{0}^{1} \sigma^{2}(v) d v
\end{aligned}
$$

because, assuming the continuous differentiability of $\sigma^{2}(\cdot)$,

$$
\sigma^{2}((t+b n) / T)-\sigma^{2}(b / \mathbf{B})=O\left(\mathbf{B}^{-1}\right), b=1, \ldots, \mathbf{B} .
$$

From here, it is standard to conclude that $\mathcal{T}_{n, \mathbf{B}}\left(\frac{\mathbf{b}^{*}}{\mathbf{B}} ; j\right)$ will have a mean given by

$$
\frac{1}{\mathbf{B}} \sum_{b=1}^{\mathbf{b}^{*}}\left\{\frac{\frac{1}{n} \sum_{t=1}^{n} \sigma^{2}((t+b n) / T)}{\int_{0}^{1} \sigma^{2}(v) d v}-1\right\} \simeq \frac{1}{\mathbf{B}} \sum_{b=1}^{\mathbf{b}^{*}} \frac{\sigma^{2}(b / \mathbf{B})}{\int_{0}^{1} \sigma^{2}(v) d v}-1 \quad \mathbf{b}^{*}=2, \ldots, \mathbf{B}
$$

which is clearly different from zero unless $\sigma_{t}^{2}=\sigma^{2}$, for all $t=1, \ldots, T$. The consequence is that the test based on $\mathcal{T} \mathcal{P}_{n, \mathbf{B}}\left(\frac{\mathbf{b}^{*}}{\mathbf{B}} ; \frac{\mathbf{j}^{*}}{\tilde{n}}\right)$ is consistent against this type of alternative. Note that (2.34) suggests that $I_{x, b}(j)$ is an unbiased estimator of $\sigma^{2}(b / \mathbf{B})$ for all $j=1, \ldots, \tilde{n}$.

\section{BOOTSTRAP}

We now focus on the bootstrap approach for our testing procedure based on $\mathcal{T} \mathcal{P}_{n, \mathbf{B}}\left(\frac{\mathbf{b}^{*}}{\mathbf{B}} ; \frac{\mathbf{j}^{*}}{\tilde{n}}\right)$ and as a by-product of $\mathcal{T}_{n, \mathbf{B}}\left(\frac{\mathbf{b}^{*}}{\mathbf{B}} ; \frac{\mathbf{j}^{*}}{\tilde{n}}\right)$. The motivation comes from the observation that our Monte Carlo experiment suggests that the asymptotic critical values may not provide a good approximation for the finite sample values. In those circumstances, the practitioner hopes that bootstrap algorithms will provide better finite sample approximations. Thus, the main aim of this section is to present a bootstrap algorithm 
and examine its validity. As usual, $\mathcal{E}^{*}$ and $\operatorname{Pr}^{*}\{\cdot\}$ indicate the expectation and the probability in the bootstrap sense, respectively.

We now describe the bootstrap. To that end, we recall that the asymptotic distribution of $[T / 2]^{1 / 2} \mathcal{T} \mathcal{P}_{n, \mathbf{B}}\left(\frac{\mathbf{b}^{*}}{\mathbf{B}} ; \frac{\mathbf{j}^{*}}{\tilde{n}}\right)$ is independent of the underlying dependence of $x_{t}$. That is, the behavior of $[T / 2]^{1 / 2} \mathcal{T} \mathcal{P}_{n, \mathbf{B}}\left(\frac{\mathbf{b}^{*}}{\mathbf{B}} ; \frac{\mathbf{j}^{*}}{\tilde{n}}\right)$ and $[T / 2]^{1 / 2} \mathcal{T}_{n, \mathbf{B}}\left(\frac{\mathbf{b}^{*}}{\mathbf{B}} ; \mathbf{j}_{\tilde{n}}^{*}\right)$ are exactly the same (asymptotically) as if we were using $\varepsilon_{t}$ instead of $x_{t}$ in their computation. So the trick of the bootstrap will not be a question of capturing or mimicking the serial dependence of the observations, but of preserving the statistical properties of the innovations. Thus, our bootstrap algorithm is based on two steps.

$\boldsymbol{S T E P} 1$ : Compute $\left\{\widehat{\varepsilon}_{t+(b-1) n}\right\}_{t=1}^{n}, b=1, \ldots, \mathbf{B}$, as in $(2.15)$ to obtain $\left\{\widetilde{\varepsilon}_{t}=\left(\widehat{\varepsilon}_{t}-\overline{\hat{\varepsilon}}\right) / \widehat{\sigma}_{\varepsilon}\right\}_{t=1}^{T}$, where

$$
\overline{\widehat{\varepsilon}}=\frac{1}{T} \sum_{t=1}^{T} \widehat{\varepsilon}_{t} ; \quad \widehat{\sigma}_{\varepsilon}^{2}=\frac{1}{T} \sum_{t=1}^{T}\left(\widehat{\varepsilon}_{t}-\overline{\widehat{\varepsilon}}\right)^{2}
$$

STEP 2: Obtain a random sample of size $T$ from the empirical distribution function of $\left\{\widetilde{\varepsilon}_{t}\right\}_{t=1}^{T}$. Denote the sample as $\left\{\varepsilon_{t}^{*}\right\}_{t=1}^{T}$, and compute the bootstrap statistic

$$
\mathcal{T} \mathcal{P}_{n, \mathbf{B}}^{*}\left(\frac{\mathbf{b}^{*}}{\mathbf{B}} ; \frac{\mathbf{j}^{*}}{\tilde{n}}\right)=\frac{1}{\tilde{n}} \sum_{j=1}^{\mathbf{j}^{*}} \frac{1}{\mathbf{B}} \sum_{b=1}^{\mathbf{b}^{*}}\left(\frac{I_{\varepsilon^{*}, b}(j) / \widehat{\sigma}_{\varepsilon^{*}}^{2}(b)}{\mathbf{B}^{-1} \sum_{v=1}^{\mathbf{B}}\left\{I_{\varepsilon^{*}, v}(j) / \widehat{\sigma}_{\varepsilon^{*}}^{2}(v)\right\}}-1\right),
$$

where $\mathbf{j}^{*}=1, \ldots, \tilde{n}$ and

$$
I_{\varepsilon^{*}, b}(j)=\left|\mathcal{J}_{\varepsilon^{*}, b}(j)\right|^{2}, \quad \widehat{\sigma}_{\varepsilon^{*}}^{2}(b)=\frac{1}{n} \sum_{t=1}^{n} \varepsilon_{t+(b-1) n}^{* 2} ; \quad b=1, \ldots, \mathbf{B}
$$

and $\mathcal{J}_{\mathcal{E}^{*}, b}(j)$ as defined in (2.9) with $z_{t}$ being replaced by $\varepsilon_{t}^{*}$ there. Then, compute the bootstrap analogues of (2.32) and (2.33), replacing $\mathcal{T} \mathcal{P}_{n, \mathbf{B}}\left(\frac{\mathbf{b}^{*}}{\mathbf{B}} ; \frac{\mathbf{j}^{*}}{\tilde{n}}\right)$ with $\mathcal{T} \mathcal{P}_{n, \mathbf{B}}^{*}\left(\frac{\mathbf{b}^{*}}{\mathbf{B}}, \frac{\mathbf{j}^{*}}{\tilde{n}}\right)$ there.

Remark 1. (a) We can replace $\widetilde{\varepsilon}_{t}$ with $\widehat{\varepsilon}_{t}$ in Step 2. The reason is that $\sum_{t=1}^{n} \overline{\widehat{\varepsilon}} e^{i t \lambda_{j}}=0$, and $I_{\varepsilon^{*}, b}(j) / \mathbf{B}^{-1} \sum_{b=1}^{\mathbf{B}} I_{\varepsilon^{*}, b}(j)$ is invariant to multiplicative constants.

(b) We may also compute $\left\{\widetilde{\varepsilon}_{t+(b-1) n}=\left(\widehat{\varepsilon}_{t+(b-1) n}-\overline{\widehat{\varepsilon}}_{b}\right) / \widehat{\sigma}_{\varepsilon, b}\right\}_{t=1}^{n}$ with

$$
\overline{\hat{\varepsilon}}_{b}=\frac{1}{n} \sum_{t=1}^{n} \widehat{\varepsilon}_{t+(b-1) n} ; \quad \widehat{\sigma}_{\varepsilon, b}^{2}=\frac{1}{n} \sum_{t=1}^{n}\left(\widehat{\varepsilon}_{t+(b-1) n}-\overline{\hat{\varepsilon}}_{b}\right)^{2}, b=1, \ldots, \mathbf{B},
$$

and obtain $\left\{\varepsilon_{t}^{*}\right\}_{t=1}^{T}=\left\{\left\{\varepsilon_{t+(b-1) n}^{*}\right\}_{t=1}^{n} ; b=1, \ldots, \mathbf{B}\right\}$, where $\left\{\varepsilon_{t+(b-1) n}^{*}\right\}_{t=1}^{n}$ is a random sample from the empirical distribution of $\left\{\widetilde{\varepsilon}_{t+(b-1) n}\right\}_{t=1}^{n}$ as in Step 2 but with random sampling within each "block" $b=1, . ., \mathbf{B}$.

We can also compute the bootstrap analogue of $\mathcal{T}_{n, \mathbf{B}}\left(\frac{\mathbf{b}^{*}}{\mathbf{B}} ; \frac{\mathbf{j}^{*}}{\tilde{n}}\right)$ as

$$
\mathcal{T}_{n, \mathbf{B}}^{*}\left(\frac{\mathbf{b}^{*}}{\mathbf{B}} ; \frac{\mathbf{j}^{*}}{\tilde{n}}\right)=\frac{1}{\tilde{n}} \sum_{j=1}^{\mathbf{j}^{*}} \frac{1}{\mathbf{B}} \sum_{b=1}^{\mathbf{b}^{*}}\left(\frac{I_{\varepsilon^{*}, b}(j)}{\mathbf{B}^{-1} \sum_{v=1}^{\mathbf{B}} I_{\varepsilon^{*}, v}(j)}-1\right) .
$$

We now have the following result on the validity of the bootstrap.

Theorem 2. Assuming $C 1$ and $C 2$, we have that
(a) $[T / 2]^{1 / 2} \mathcal{T}_{n, \mathbf{B}}^{*}\left(\frac{\mathbf{b}^{*}}{\mathbf{B}} ; \frac{\mathbf{j}^{*}}{\tilde{n}}\right) \stackrel{\text { weakly }}{\Rightarrow} \mathcal{B S}\left([0,1]^{2} ; \kappa_{4}\right) \quad$ (in probability)
(b) $[T / 2]^{1 / 2} \mathcal{T P}_{n, \mathbf{B}}^{*}\left(\frac{\mathbf{b}^{*}}{\mathbf{B}} ; \frac{\mathbf{j}^{*}}{\tilde{n}}\right) \stackrel{\text { weakly }}{\Rightarrow} \mathcal{W B}\left([0,1]^{2}\right) \quad$ (in probability). 
From Theorem 2 and the continuous mapping theorem we have that for any continuous functional $\varphi(\cdot)$
(a) $\varphi\left([T / 2]^{1 / 2} \mathcal{T}_{n, \mathbf{B}}^{*}\left(\frac{\mathbf{b}^{*}}{\mathbf{B}} ; \frac{\mathbf{j}^{*}}{\tilde{n}}\right)\right) \stackrel{\text { weakly }}{\Rightarrow} \varphi\left(\mathcal{B S}\left([0,1]^{2} ; \kappa_{4}\right)\right)$
(in probability)
(b) $\varphi\left([T / 2]^{1 / 2} \mathcal{T} \mathcal{P}_{n, \mathbf{B}}^{*}\left(\frac{\mathbf{b}^{*}}{\mathbf{B}} ; \frac{\mathbf{j}^{*}}{\tilde{n}}\right)\right) \stackrel{\text { weakly }}{\Rightarrow} \varphi\left(\mathcal{W B}\left([0,1]^{2}\right)\right)$
(in probability).

We finish the section indicating that Theorem 2 part (b) holds true under Conditions $C 1^{\prime}, C 2^{\prime}$ and $C 2$. On the other hand, part $(a)$ holds true except that the bootstrap statistic will converge in probability to $\mathcal{B S}\left([0,1]^{2} ; \kappa_{4}^{\dagger}\right)$, where $\kappa_{4}^{\dagger}=p \lim _{T \rightarrow \infty} \widehat{\kappa}_{4}$ with $\widehat{\kappa}_{4}$ given in $(2.16)$.

\section{MONTE CARLO EXPERIMENT: EMPIRICAL EXAMPLES}

The purpose of this section is to present a Monte Carlo experiment to shed some light on the finite sample performance of the bootstrapped version of our preferred test $\mathcal{T} \mathcal{P}_{n, \mathbf{B}}$. We consider sample sizes of $T=256,512$ and 1024. For each combination of $T, n$ and models considered in the experiment, we conduct 1,000 simulation runs. To save computational time, for each run, we compute only one bootstrap counterpart. The bootstrapped distribution is obtained by stacking those statistics across iterations, and then used to construct critical values and confidence regions at the desired levels. This is the idea behind the WARP algorithm of Giacomini et al. (2013).

\subsection{Level considerations and choice of block sizes.}

We study the nominal level of the bootstrapped modified statistic, given in equation (b) of Theorem 3 , with the following $A R M A(2,1)$ model. We simulate

$$
x_{t, T}=\phi_{1} x_{t-1, T}+\phi_{2} x_{t-2, T}+\varepsilon_{t, T}+\theta \varepsilon_{t-1, T}
$$

for several combinations of the parameter vector $\left(\phi_{1}, \phi_{2}, \theta\right)$. In all specifications, $\varepsilon_{t} \sim \mathcal{N} \mathcal{I D}(0,1)$. Model (4.1) nests models (4.2)-(4.4) considered by Dette et al. (2011) and implemented as models (4.2)-(4.3) by Preuß et al. (2013). For the sake of comparison, their tests are also replicated here.

The $A R(1)$ model corresponds to $\phi_{2}=\theta=0$. The results for various $\phi_{1}$ are reported in Table $1 .{ }^{1}$ Even for small sample sizes such as $T=256$, the rejection probabilities are close to the $5 \%$ and $10 \%$ values. As $T$ increases, the size distortions become very small, especially when $\left|\phi_{1}\right|$ is close to zero. The behavior of the Cramér-von-Mises $(\mathcal{C} v \mathcal{M})$ and Komolgorov-Smirnov $(\mathcal{K S})$ functionals appear to be similar. While neither statistic clearly dominates the other, the latter emerges slightly more robust for small values of the parameters. ${ }^{2}$ Since the choice of block size is an inherent aspect of the test, ${ }^{3}$ we examine the sensitivity of the test to that choice by computing the test for a grid of values for $n$. For that purpose, we consider three values of $n$ for each sample size $T{ }^{4}$ It seems that the best performance for $T=256$ is $n=32$; for $T=512, n=32$; and finally for $T=1024, n=128$. For the sake of clarity, in the tables that follow, we have marked those pairs with the " $\triangleright$ " sign. It appears that the test is not too sensitive to the choice of $n .^{5}$

\footnotetext{
${ }^{1}$ Table 4 in the appendix presents rejection probabilities of the null hypothesis of weak stationarity of the non-bootstrapped statistics.

${ }^{2}$ We also present the distribution of the non-bootstrapped statistics in Table 4 . As previously mentioned, at small sample sizes, the asymptotic distribution does not provide a reasonable approximation.

${ }^{3}$ For example, Dette et al. (2011, p. 1118) remark that “(..) any statistical inference in locally stationary process depend on the choice of [the parameters] in the definition of the local periodogram."

${ }^{4}$ In all cases, we limit ourselves to sample and block sizes with length of powers of 2 . This allows more efficient computation of fast Fourier transform algorithms.

${ }^{5}$ Block size $n$ can also be chosen by minimizing the integrated mean squared error or, alternatively, by cross-validation.
} 
Table 2 reports rejection probabilities for different values of the parameters of model (4.1). Assuming $\phi_{1}=\phi_{2}=0.3$ and $\theta=0$, we obtain an autoregressive process with real roots; the process at parameters $\phi_{1}=0.4, \phi_{2}=-0.3$ and $\theta=0$ exhibits complex roots. The remaining cases contemplate two $A R M A(1,1)$ and one $A R M A(2,1)$ processes. Size is again well-approximated at small sample sizes, block sizes and parameter values.

Overall, the Monte Carlo results show that size distortions for the $\mathcal{C} v \mathcal{M}$ and $\mathcal{K} \mathcal{S}$ tests are quite small and they have comparable performance. We observe, however, very significant size distortions by Preuß et al. $(2013)$ at the set of parameters $\left(\phi_{1}, \phi_{2}, \theta\right)=(0.4,-0.7,0),(0.5,0,0.5)$ and $(0.3,0.3,0.5)$.

\subsection{Power considerations.}

We study the power performance of the test with recourse to the following five data-generating processes (DGPs)

$$
\begin{aligned}
& x_{t, T}=\left\{\begin{array}{l}
0.2 x_{t-1, T}+e_{t, T}, \quad t=2, \ldots, \frac{T}{2} \\
0.7 x_{t-1, T}+e_{t, T}, \quad t=\frac{T}{2}+1, \ldots, T
\end{array}\right. \\
& x_{t, T}= \begin{cases}0.4 x_{t-1, T}-0.7 x_{t-2, T}+e_{t, T}, & t=2, \ldots, \frac{T}{2} \\
0.3 x_{t-1, T}+0.3 x_{t-2, T}+e_{t, T}, & t=\frac{T}{2}+1, \ldots, T\end{cases} \\
& x_{t, T}= \begin{cases}0.3 x_{t-1, T}+0.3 x_{t-2, T}+e_{t, T}, & t=2, \ldots, \frac{T}{2} \\
0.8 x_{t-1, T}+e_{t, T}, & t=\frac{T}{2}+1, \ldots, T\end{cases} \\
& x_{t, T}=0.6 \sin (4 \pi t / T) x_{t-1, T}+e_{t, T} \\
& x_{t, T}=1.1 \cos (1.5-\cos (4 \pi t / T)) e_{t-1, T}+e_{t, T}
\end{aligned}
$$

The first DGP is a break of the AR(1) coefficient. The break in the second DGP is such that the roots switch from complex to real. The third model considers a change in the order of the autoregressive model. Finally, the fourth and fifth models have changing coefficients with $t$ and originating from Dette et al. (2011), equations (4.6) and (4.7).

In Table 3, we observe that deviation from the null hypothesis is detected at a reasonable frequency, which increases quickly as $T$ grows. For $T=2048$, the rejection probabilities are either very close to one or the rejection of the null is obtained in every simulation run. At smaller sample sizes, power is naturally higher for those DGPs that impose a large change in the spectral density functions, particularly (4.3). The $\mathcal{K S}$ functional shows slightly higher power than $\mathcal{C} v \mathcal{M}$ in small sample sizes, particularly DGPs (4.2) and (4.4).

For data generated according to DGP (4.2), Dette et al. (2011) achieves comparable power relative to $\mathcal{C} v \mathcal{M}$, followed by the $\mathcal{K S}$ functional; Preuß et al. (2013) obtains higher power at small sample sizes. A similar pattern is observed in DGP (4.4). The ordering, however, appears inverted for DGP (4.3) and approximately so for (4.5) and (4.6). We note that our test achieves reasonable power for all data-generating processes and more stable results compared to the tests above.

We summarize the size and power simulation results in Figure 1. The complete set of results is shown in Appendix Tables 1-3.

4.3. Empirical examples. We implement the proposed test to check the stability of the dynamics on two real data sets. In our first application, we present evidence that inflation dynamics are not constant over time in the United States. We use data on the baseline Consumer Price Index (CPI) obtained 
Figure 1. Rejection probabilities for $\mathcal{C} v \mathcal{M}, \mathcal{K S}$, Dette et al. (2011) and Preuss et al. (2013) statistics
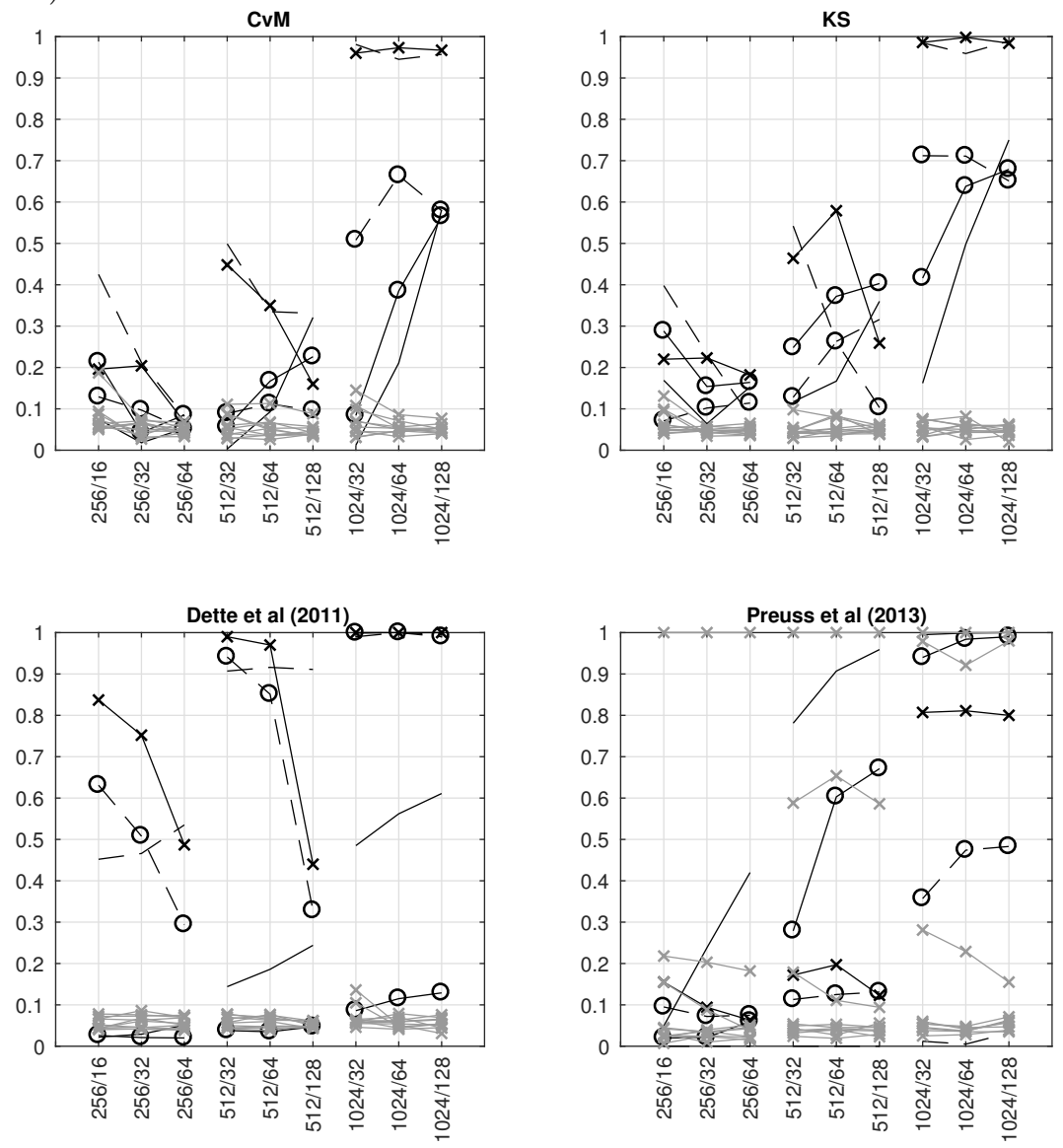

$$
{ }^{(4.3)} \quad-\text { (4.4) }^{(4.4)} \boldsymbol{O}^{(4.5)} \quad \mathrm{O}^{(4.6)} \quad \mathrm{x}^{(4.7)}
$$

Note: Rejection probabilities for several combinations of $T$ and $n$, represented by the notation $T / n$. DGPs implemented for size assessments are grayed out, and DGPs (4.3)-(4.7) for power assessments are individually labelled.

by the U.S. Bureau of Labour Statistics, between September 1959 and August 2015, for a total of 768 observations.

Our second application replicates the neuroscience example of Dette et al. (2011), also used by von Sachs and Neumann (2000) and Paparoditis (2009). ${ }^{6}$ The authors analyze a data set of tremor activities recorded in the Cognitive Neuroscience Laboratory of the University of Quebec at Montreal from subjects with Parkinson's Disease. The aim is to compare different regions of patients' brain activity. The data has 3,072 observations.

To guarantee weak stationarity for both sequences, we take first differences $\Delta x_{t, T}=x_{t, T}-x_{t-1, T}$. Figure 2 presents the smoothed spectral density estimate

$$
\hat{f}(u ; \lambda)=\frac{2 \pi}{n} \sum_{j=1}^{n} \frac{1}{k} K\left(\frac{\lambda-\lambda_{j}}{k}\right) I_{x, b}(j),
$$

\footnotetext{
${ }^{6}$ We thank Efstathios Paparoditis for sharing these data.
} 
Figure 2. Estimates of local spectral density for Consumer Price Index (left) and tremor data (right)

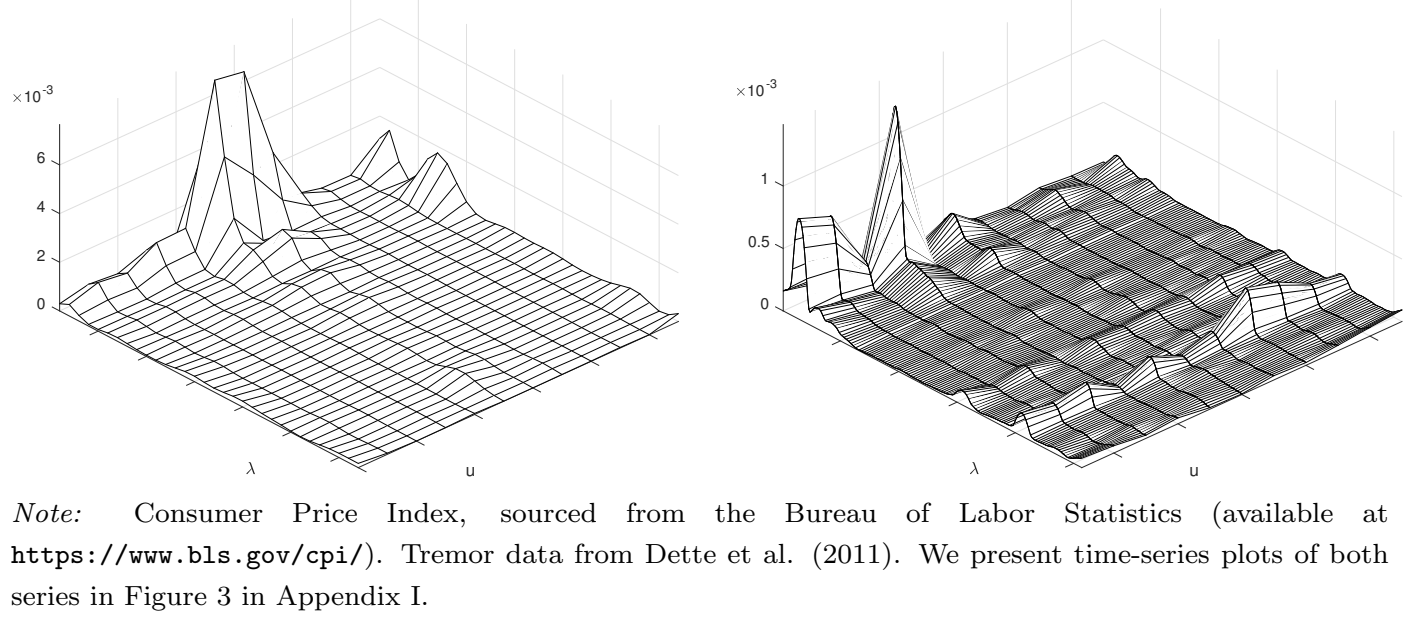

where $K(\cdot)$ denotes the Bartlett-Priestley kernel. A similar approach is introduced by Dette et al. (2011) and Paparoditis (2009). For the CPI application, we choose $n=64$ and $k=0.01$, whereas for the neuroscience data, we employ $n=256$ and $k=0.18 .^{7}$ The figures strongly suggest that the spectral densities vary over time, especially at lower frequencies.

In Table 5 we present the Cramér-von-Mises $(\mathcal{C} v \mathcal{M})$ and Kolmogorov-Smirnov $(\mathcal{K S})$ test statistics for the CPI data, along with the bootstrapped $10 \%, 5 \%$ and $1 \%$ critical values. ${ }^{8}$ The block sizes considered are in line with simulations presented in the previous subsection. In all cases, the null hypothesis is rejected at the $1 \%$ level, with the outcome of the test not being sensitive to the choice of the bandwidth parameter and the $p$-value being clearly less than $1 \%$.

For the neuroscience application, in line with Dette et al. (2011) and others, we reject the null hypothesis at the $1 \%$ level. Bootstrapped critical values and test statistics are presented in Table 6 .

\section{CONCLUSION}

In this paper, we describe and examine a simple test for the hypothesis of stable dynamics without assuming any parametric family under the null hypothesis. One interesting aspect of the test is that, even without knowledge of the spectral density function under the null hypothesis, there is no need to choose any bandwidth or smoothing parameter for its implementation, besides the choice of the length of the block size $n$. A second interesting aspect of the test is that its asymptotic distribution is pivotal. We also describe a modification of the test such that its asymptotic behavior only depends on $\kappa_{4}$. Based on the canonical decomposition of the spectral density function as given by Whittle (1963), see also Hannan (1970) or Brillinger (1981), we examine a consistent estimator of $\kappa_{4}$. In addition, the test does not require any type of "bias" adjustments for its implementation, and it is able to detect local alternatives converging to the null hypothesis at the parametric rate $T^{-1 / 2}$.

\footnotetext{
${ }^{7}$ Again, this is done following Dette et al. (2011) and Paparoditis (2009).

${ }^{8}$ We use 500 iterations in each case.
} 


\section{REFERENCES}

[1] Anderson, T.W. and Walker, A.M. (1964): "On the asymptotic distribution of the autocorrelations of a sample from a linear stochastic process," Annals of Mathematical Statistics, 35, 1296-1303.

[2] Aue, A. and Horvarth, L. (2013): "Structural breaks in time series," Journal of Time Series Analysis , 34, 1-16.

[3] Bickel, P.J. and Wichura, M.J. (1971): "Convergence criteria for multiparameter stochastic processes and its applications," Annals of Mathematical Statistics, 42, 1656-1670.

[4] Bandyopadhy, A.Y., Jentsch, C. and Subba Rao, S. (2017): "Spectral domain test for stationarity of spatiotemporal data," Journal of Time Series Analysis, 38, 326-351.

[5] Brillinger, D.R. (1981): Time Series, Data Analysis and Theory. Holden-Day, San Francisco.

[6] Brockwell, P.J. And Davis, R.A. (1991): Time Series: Theory and Methods. Springer-Verlag, New York.

[7] Brown, R.L., Durbin, J. and Evans, J.M. (1975): "Techniques for testing constancy of regression relationships over time (with discussion)," Journal of the Royal Statistical Society: Ser. B., 37, 149-192.

[8] Dahlhaus, R. (1996): "On the Kulback-Leibler information divergence of locally stationary processes," Stochastic Processes and its Applications, 62, 139-168.

[9] Dahlhaus, R. (1997): "Fitting time series models to nonstationary processes," Annals of Statistics, 25, 1-37.

[10] Dahlhaus, R. (2009): "Local inference for locally stationary time series based on the empirical spectral measure," Journal of Econometrics, 151, 101-112.

[11] Dahlhaus, R. And Janas, D. (1996): "A frequency domain bootstrap for ratio statistics in time series analysis," Annals of Statistics, 24, 1934-1963.

[12] Dahlhaus, R. and Polonik, W. (2009): "Empirical spectral processes for locally stationary time series," Bernoulli, 15, 1-39.

[13] Dalla, V., Giraitis, L. and Hidalgo, J. (2005): "Consistent estimation of the memory parameter for nonlinear time series," Journal of Time Series Analysis, 27, 211-255.

[14] Davis, R.A., Huang, D. and Yao, Y. (1995): "Testing for a Change in the Parameter Values and Order of an Autoregressive Model," Annals of Statistics, 23, 282-304.

[15] Delgado, M.A., Hidalgo, J. and Velasco, C. (2005): "Distribution free Goodness-of-fit tests for linear processes," Annals of Statistics, 33, 2568-2609.

[16] Dette, H., Preuss, P., And Vetter, M. (2011): "A measure of stationarity in locally stationary processes with applications to testing," Journal of the American Statistical Association, 106:495, 1113-1124.

[17] Dwivedi, Y., and Subba Rao, S. (2011): "A test for second-order stationarity of a time series based on the discrete Fourier transform," Journal of Time Series Analysis, 32, 68-91.

[18] Fragkeskou, M. and Paparoditis, E. (2016): "Inference for the fourth-order innovation cumulant in linear time series," Journal of Time Series Analysis, 37, 240-266.

[19] Giacomini, R., Politis, D.N. And White, H. (2013): "A warp-speed method for conducting Monte Carlo experiments involving bootstrap estimators," Econometric Theory, 29, 567-589.

[20] Grenander, U. and Rosenblatt, M. (1957): Statistical Analysis of Stationary Time Series. John Wiley, New York.

[21] Hannan, E.J. (1970). Multiple Time Series. Wiley and Sons, New York.

[22] Härdle, W., Mammen, E. (1993): "Comparing nonparametric versus parametric regression fits," Annals of Statistics, 21, 1926-1947.

[23] Hidalgo, J. (2007): "Specification testing for regression models with dependent data," Journal of Econometrics, 143, $143-163$.

[24] Hidalgo, J. And Seo, M.(2015): "Specification with lattice processes," Econometric Theory, 31, 294-336.

[25] Ibragimov, I.A. And Rozanov, Y.A. (1978). Gaussian random processes. Springer-Verlag. Berlin.

[26] Jentsch, C. and Subba Rao, S. (2015): "A test for second order stationarity of a multivariate time series," Journal of Econometrics, 185, 124-161.

[27] Lee, S., HA, J., NA, O. And NA, S. (2003): "The Cusum Test for Parameter Change in Time Series Models," Scandinavian Journal of Statistics, 30, 781-796.

[28] Lucas, R.E. (1976): "Econometric Policy Evaluation: A Critique," Carnegie-Rochester Conference Series on Public Policy Vol. 1, 19-46.

[29] Paparoditis, E. (2009): "Testing temporal constancy of the spectral structure of a time series," Bernoulli, 15, 11901221. 
[30] Perron, P. (2006): "Dealing with structural breaks," Pelgrave Handbook of Econometrics, Vol. 1: Econometric Theory.

[31] Picard, D. (1985): "Testing and estimating change points in time series," Advances in Time Series Analysis, 17, $841-867$

[32] Preuss, P., Vetter, M. And Dette, H. (2013): "A test for stationarity based on empirical processes," Bernoulli, 19, 2715-1749.

[33] Priestley, M.B. (1965): "Evolutionary spectra and non-stationary processes," Journal of the Royal Statistical Society, Ser. B, 62, 204-237.

[34] von Sachs, R., And Neumann, M. H. (2000): “A wavelet-based test for stationarity," Journal of Time Series Analysis, 21:5, 597-613.

[35] Welch, P.D. (1967): "The use of fast Fourier Transform for the estimation of power spectra: A method based on time averaging over short, modified periodograms," IEEE Transaction of Audio and Electroacoustic, 15, 70-73.

[36] Whittle, P. (1963): Prediction and Regulation. Van Nostrand, Princeton: New Jersey. 
APPENDIX I: TABLES AND FIGURES

TABLE 1. Rejection probabilities (size), model (4.1) with $\phi_{2}=\theta=0$

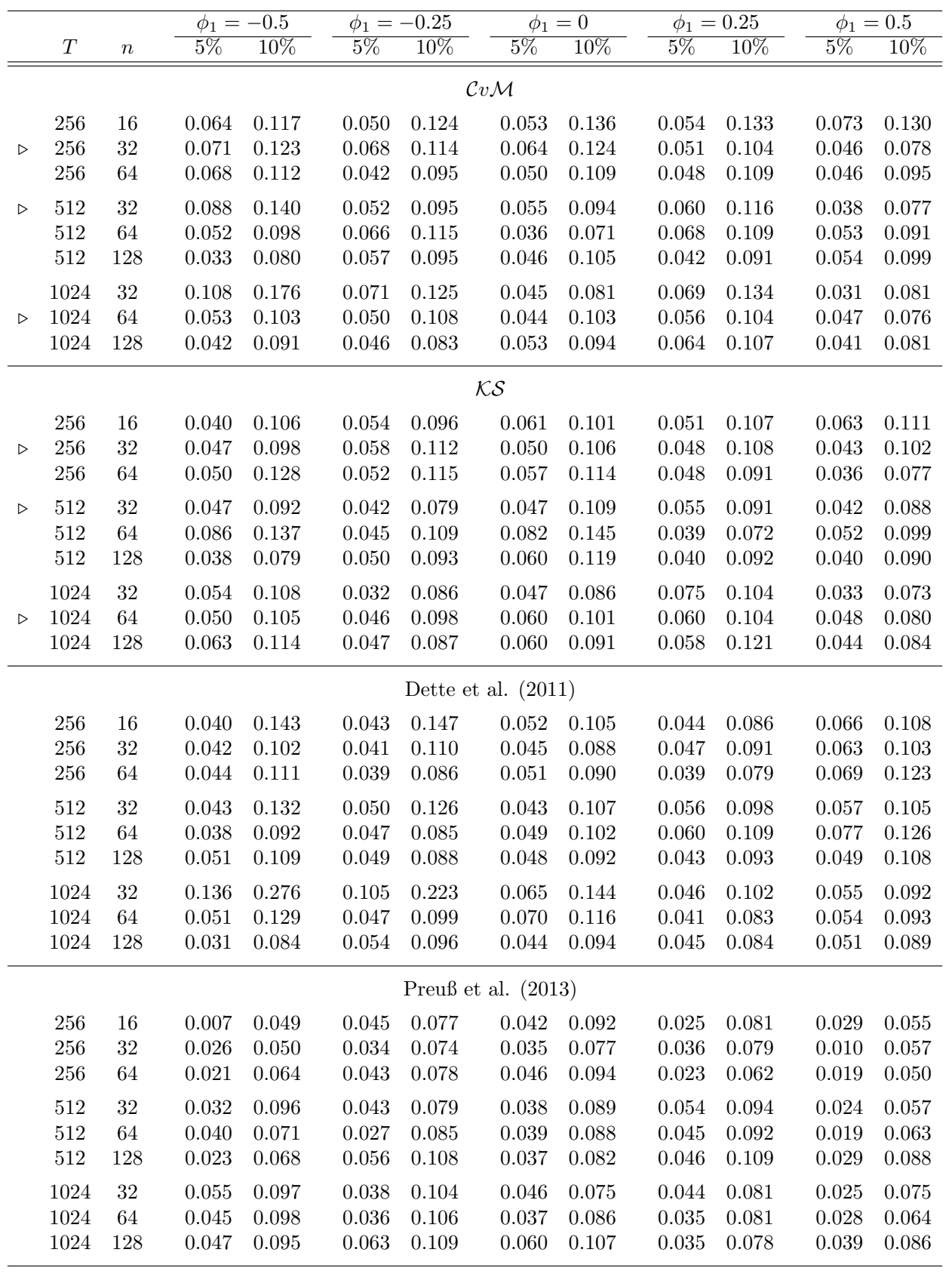


TABLE 2. Rejection probabilities (size), model (4.1), various parameters

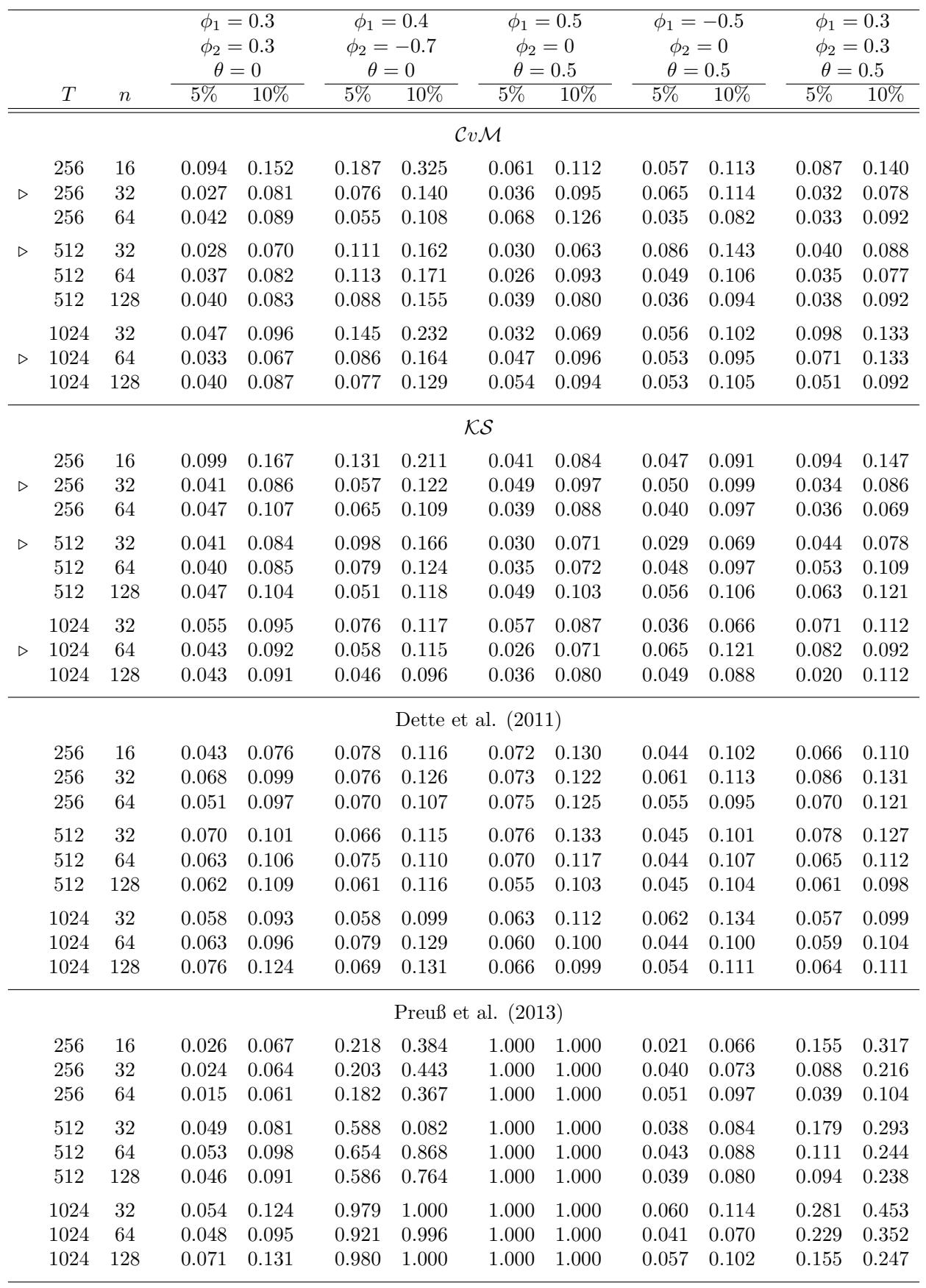


TABLE 3. Rejection probabilities (power), models (4.2)- (4.6)

\begin{tabular}{|c|c|c|c|c|c|c|c|c|c|c|c|c|}
\hline & \multirow[b]{2}{*}{$T$} & \multirow[b]{2}{*}{$n$} & \multicolumn{2}{|c|}{$(4.2)$} & \multicolumn{2}{|c|}{$(4.3)$} & \multicolumn{2}{|c|}{$(4.4)$} & \multicolumn{2}{|c|}{$(4.5)$} & \multicolumn{2}{|c|}{$(4.6)$} \\
\hline & & & $5 \%$ & $10 \%$ & $5 \%$ & $10 \%$ & $5 \%$ & $10 \%$ & $5 \%$ & $10 \%$ & $5 \%$ & $10 \%$ \\
\hline \multicolumn{13}{|c|}{$\mathcal{C} v \mathcal{M}$} \\
\hline \multirow{3}{*}{$\triangleright$} & 256 & 16 & 0.075 & 0.164 & 0.425 & 0.571 & 0.213 & 0.297 & 0.129 & 0.192 & 0.196 & 0.303 \\
\hline & 256 & 32 & 0.018 & 0.053 & 0.212 & 0.311 & 0.043 & 0.127 & 0.097 & 0.167 & 0.204 & 0.359 \\
\hline & 256 & 64 & 0.053 & 0.112 & 0.082 & 0.149 & 0.085 & 0.188 & 0.051 & 0.121 & 0.068 & 0.125 \\
\hline \multirow[t]{4}{*}{$\triangleright$} & 512 & 32 & 0.003 & 0.050 & 0.499 & 0.683 & 0.056 & 0.150 & 0.089 & 0.193 & 0.448 & 0.628 \\
\hline & 512 & 64 & 0.093 & 0.227 & 0.335 & 0.539 & 0.167 & 0.297 & 0.112 & 0.216 & 0.350 & 0.563 \\
\hline & 512 & 128 & 0.321 & 0.488 & 0.331 & 0.573 & 0.226 & 0.421 & 0.096 & 0.223 & 0.160 & 0.361 \\
\hline & 1024 & 32 & 0.012 & 0.136 & 0.981 & 0.991 & 0.084 & 0.326 & 0.508 & 0.700 & 0.960 & 0.987 \\
\hline \multirow[t]{2}{*}{$\triangleright$} & 1024 & 64 & 0.211 & 0.396 & 0.945 & 0.996 & 0.385 & 0.591 & 0.664 & 0.770 & 0.973 & 0.997 \\
\hline & 1024 & 128 & 0.581 & 0.827 & 0.957 & 0.998 & 0.565 & 0.762 & 0.579 & 0.775 & 0.967 & 0.992 \\
\hline \multirow[t]{3}{*}{$\triangleright$} & 2048 & 64 & 0.691 & 0.937 & 1.000 & 1.000 & 0.642 & 0.844 & 0.995 & 0.998 & 1.000 & 1.000 \\
\hline & 2048 & 128 & 0.972 & 0.997 & 1.000 & 1.000 & 0.772 & 0.964 & 0.986 & 1.000 & 1.000 & 1.000 \\
\hline & 2048 & 256 & 0.997 & 1.000 & 1.000 & 1.000 & 0.974 & 0.996 & 0.987 & 0.998 & 1.000 & 1.000 \\
\hline \multicolumn{13}{|c|}{$\mathcal{K S}$} \\
\hline \multirow{3}{*}{$\triangleright$} & 256 & 16 & 0.169 & 0.252 & 0.398 & 0.585 & 0.288 & 0.415 & 0.071 & 0.204 & 0.220 & 0.424 \\
\hline & 256 & 32 & 0.064 & 0.118 & 0.232 & 0.378 & 0.154 & 0.252 & 0.102 & 0.206 & 0.223 & 0.441 \\
\hline & 256 & 64 & 0.153 & 0.241 & 0.082 & 0.156 & 0.164 & 0.266 & 0.114 & 0.246 & 0.182 & 0.350 \\
\hline \multirow[t]{4}{*}{$\triangleright$} & 512 & 32 & 0.117 & 0.232 & 0.542 & 0.736 & 0.248 & 0.374 & 0.128 & 0.288 & 0.464 & 0.665 \\
\hline & 512 & 64 & 0.167 & 0.349 & 0.263 & 0.551 & 0.372 & 0.530 & 0.262 & 0.394 & 0.579 & 0.714 \\
\hline & 512 & 128 & 0.360 & 0.551 & 0.316 & 0.549 & 0.403 & 0.584 & 0.103 & 0.254 & 0.259 & 0.496 \\
\hline & 1024 & 32 & 0.162 & 0.441 & 0.985 & 1.000 & 0.416 & 0.651 & 0.712 & 0.881 & 0.986 & 0.997 \\
\hline \multirow[t]{2}{*}{$\triangleright$} & 1024 & 64 & 0.498 & 0.718 & 0.959 & 0.998 & 0.638 & 0.800 & 0.711 & 0.903 & 0.998 & 1.000 \\
\hline & 1024 & 128 & 0.750 & 0.907 & 0.989 & 1.000 & 0.679 & 0.831 & 0.651 & 0.797 & 0.984 & 0.998 \\
\hline \multirow[t]{3}{*}{$\triangleright$} & 2048 & 64 & 0.799 & 0.948 & 1.000 & 1.000 & 0.978 & 0.994 & 0.995 & 1.000 & 1.000 & 1.000 \\
\hline & 2048 & 128 & 0.978 & 1.000 & 1.000 & 1.000 & 0.982 & 1.000 & 1.000 & 1.000 & 1.000 & 1.000 \\
\hline & 2048 & 256 & 0.998 & 1.000 & 1.000 & 1.000 & 0.990 & 1.000 & 1.000 & 1.000 & 1.000 & 1.000 \\
\hline \multicolumn{13}{|c|}{ Dette et al. (2011) } \\
\hline & 256 & 16 & 0.022 & 0.107 & 0.452 & 0.710 & 0.026 & 0.085 & 0.631 & 0.817 & 0.837 & 0.919 \\
\hline & 256 & 32 & 0.029 & 0.125 & 0.466 & 0.736 & 0.021 & 0.066 & 0.508 & 0.714 & 0.752 & 0.862 \\
\hline & 256 & 64 & 0.051 & 0.172 & 0.535 & 0.759 & 0.020 & 0.071 & 0.294 & 0.489 & 0.487 & 0.675 \\
\hline & 512 & 32 & 0.144 & 0.354 & 0.907 & 0.976 & 0.038 & 0.142 & 0.941 & 0.973 & 0.990 & 0.998 \\
\hline & 512 & 64 & 0.186 & 0.388 & 0.916 & 0.997 & 0.035 & 0.119 & 0.851 & 0.932 & 0.970 & 0.986 \\
\hline & 512 & 128 & 0.244 & 0.460 & 0.911 & 0.978 & 0.047 & 0.147 & 0.328 & 0.514 & 0.440 & 0.600 \\
\hline & 1024 & 32 & 0.485 & 0.705 & 0.990 & 1.000 & 0.086 & 0.227 & 0.999 & 1.000 & 1.000 & 1.000 \\
\hline & 1024 & 64 & 0.562 & 0.772 & 1.000 & 1.000 & 0.115 & 0.285 & 1.000 & 1.000 & 1.000 & 1.000 \\
\hline & 1024 & 128 & 0.611 & 0.796 & 0.996 & 1.000 & 0.129 & 0.294 & 0.990 & 1.000 & 1.000 & 1.000 \\
\hline & & & & & & Preuß & l. (201 & & & & & \\
\hline & 256 & 16 & 0.049 & 0.160 & 0.000 & 0.020 & 0.020 & 0.028 & 0.095 & 0.187 & 0.155 & 0.210 \\
\hline & 256 & 32 & 0.238 & 0.433 & 0.000 & 0.000 & 0.021 & 0.154 & 0.072 & 0.134 & 0.094 & 0.177 \\
\hline & 256 & 64 & 0.420 & 0.590 & 0.000 & 0.000 & 0.060 & 0.190 & 0.075 & 0.126 & 0.063 & 0.138 \\
\hline & 512 & 32 & 0.781 & 0.890 & 0.000 & 0.060 & 0.279 & 0.583 & 0.113 & 0.234 & 0.172 & 0.307 \\
\hline & 512 & 64 & 0.907 & 0.949 & 0.000 & 0.000 & 0.603 & 0.754 & 0.125 & 0.203 & 0.197 & 0.310 \\
\hline & 512 & 128 & 0.959 & 0.984 & 0.000 & 0.012 & 0.671 & 0.853 & 0.131 & 0.225 & 0.123 & 0.219 \\
\hline & 1024 & 32 & 0.995 & 0.999 & 0.012 & 0.243 & 0.940 & 0.972 & 0.357 & 0.539 & 0.807 & 0.884 \\
\hline & 1024 & 64 & 0.999 & 0.999 & 0.005 & 0.178 & 0.984 & 0.995 & 0.474 & 0.626 & 0.811 & 0.931 \\
\hline & 1024 & 128 & 1.000 & 1.000 & 0.032 & 0.224 & 0.990 & 0.994 & 0.483 & 0.739 & 0.800 & 0.900 \\
\hline
\end{tabular}


TABLE 4. Rejection probabilities (size), non-bootstrapped $\mathcal{C} v \mathcal{M}$ and $\mathcal{K S}$ statistics, model (4.1) with $\phi_{2}=\theta=0$

\begin{tabular}{|c|c|c|c|c|c|c|c|c|c|c|c|}
\hline \multirow[b]{2}{*}{$T$} & \multirow[b]{2}{*}{$n$} & \multicolumn{2}{|c|}{$\phi_{1}=-0.5$} & \multicolumn{2}{|c|}{$\phi_{1}=-0.25$} & \multicolumn{2}{|c|}{$\phi_{1}=0$} & \multicolumn{2}{|c|}{$\phi_{1}=0.25$} & \multicolumn{2}{|c|}{$\phi_{1}=0.5$} \\
\hline & & $5 \%$ & $10 \%$ & $5 \%$ & $10 \%$ & $5 \%$ & $10 \%$ & $5 \%$ & $10 \%$ & $5 \%$ & $10 \%$ \\
\hline \multicolumn{12}{|c|}{$\mathcal{C} v \mathcal{M}$} \\
\hline 256 & 32 & 0.029 & 0.045 & 0.030 & 0.040 & 0.039 & 0.049 & 0.029 & 0.045 & 0.035 & 0.047 \\
\hline 256 & 64 & 0.030 & 0.040 & 0.017 & 0.022 & 0.021 & 0.026 & 0.018 & 0.023 & 0.020 & 0.026 \\
\hline 256 & 128 & 0.008 & 0.017 & 0.014 & 0.021 & 0.012 & 0.016 & 0.014 & 0.021 & 0.016 & 0.020 \\
\hline 512 & 64 & 0.069 & 0.098 & 0.099 & 0.119 & 0.077 & 0.104 & 0.092 & 0.122 & 0.092 & 0.125 \\
\hline 512 & 128 & 0.056 & 0.081 & 0.053 & 0.069 & 0.071 & 0.087 & 0.054 & 0.069 & 0.053 & 0.070 \\
\hline 512 & 256 & 0.034 & 0.047 & 0.037 & 0.043 & 0.030 & 0.043 & 0.038 & 0.043 & 0.039 & 0.046 \\
\hline 1024 & 128 & 0.167 & 0.213 & 0.184 & 0.232 & 0.167 & 0.197 & 0.183 & 0.234 & 0.186 & 0.236 \\
\hline 1024 & 256 & 0.132 & 0.163 & 0.135 & 0.162 & 0.133 & 0.162 & 0.138 & 0.159 & 0.136 & 0.167 \\
\hline 1024 & 512 & 0.079 & 0.097 & 0.083 & 0.099 & 0.094 & 0.111 & 0.086 & 0.103 & 0.086 & 0.101 \\
\hline \multicolumn{12}{|c|}{$\mathcal{K S}$} \\
\hline 256 & 16 & 0.057 & 0.118 & 0.046 & 0.098 & 0.046 & 0.092 & 0.048 & 0.098 & 0.060 & 0.114 \\
\hline 256 & 32 & 0.023 & 0.049 & 0.021 & 0.043 & 0.027 & 0.061 & 0.020 & 0.039 & 0.020 & 0.047 \\
\hline 256 & 64 & 0.012 & 0.035 & 0.014 & 0.031 & 0.013 & 0.024 & 0.015 & 0.031 & 0.018 & 0.033 \\
\hline 512 & 32 & 0.066 & 0.117 & 0.057 & 0.107 & 0.045 & 0.090 & 0.063 & 0.112 & 0.064 & 0.123 \\
\hline 512 & 64 & 0.025 & 0.065 & 0.025 & 0.062 & 0.026 & 0.061 & 0.023 & 0.058 & 0.026 & 0.058 \\
\hline 512 & 128 & 0.008 & 0.028 & 0.008 & 0.029 & 0.007 & 0.026 & 0.011 & 0.032 & 0.013 & 0.033 \\
\hline 1024 & 64 & 0.070 & 0.159 & 0.059 & 0.141 & 0.062 & 0.128 & 0.063 & 0.136 & 0.074 & 0.143 \\
\hline 1024 & 128 & 0.051 & 0.105 & 0.048 & 0.095 & 0.054 & 0.107 & 0.044 & 0.106 & 0.052 & 0.110 \\
\hline 1024 & 256 & 0.033 & 0.073 & 0.035 & 0.071 & 0.031 & 0.067 & 0.037 & 0.070 & 0.031 & 0.069 \\
\hline
\end{tabular}

Figure 3. CPI (left) and tremor data (right)
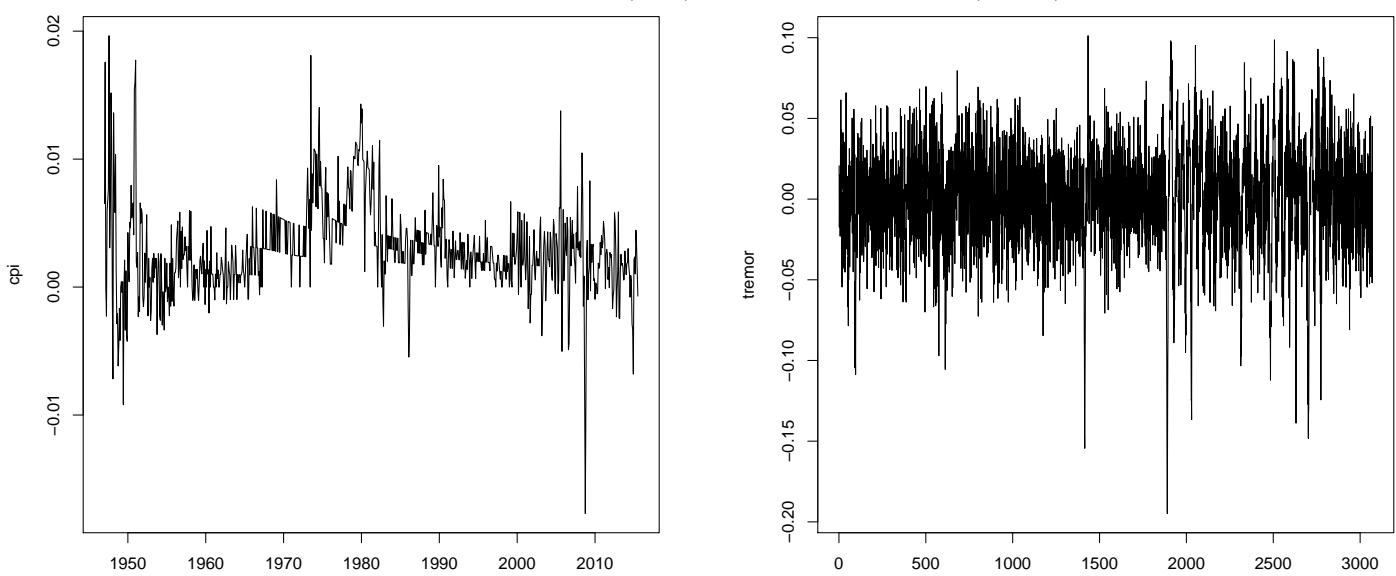

TABLE 5. U.S. Inflation Rate

\begin{tabular}{ccccccc}
\hline & $n$ & TS & $p$-value & $10 \%$ & $5 \%$ & $1 \%$ \\
\hline \hline $\mathcal{C} v \mathcal{M}$ & 32 & 2.958 & $<0.002$ & 1.043 & 1.147 & 1.340 \\
$\mathcal{C} v \mathcal{M}$ & 64 & 3.363 & $<0.002$ & 1.137 & 1.246 & 1.501 \\
$\mathcal{C} v \mathcal{M}$ & 128 & 3.264 & $<0.002$ & 1.057 & 1.144 & 1.315 \\
$\mathcal{K S}$ & 32 & 29.055 & $<0.002$ & 2.510 & 2.958 & 5.281 \\
$\mathcal{K S}$ & 64 & 39.683 & $<0.002$ & 3.227 & 4.361 & 6.802 \\
$\mathcal{K} \mathcal{S}$ & 128 & 35.676 & $<0.002$ & 3.023 & 3.904 & 6.067 \\
\hline
\end{tabular}


TABLE 6. Neuroscience Data

\begin{tabular}{ccccccc}
\hline & $n$ & TS & $p$-value & $10 \%$ & $5 \%$ & $1 \%$ \\
\hline $\mathcal{\mathcal { C }} v \mathcal{M}$ & 64 & 2.059 & $<0.002$ & 1.279 & 1.345 & 1.597 \\
$\mathcal{C} v \mathcal{M}$ & 128 & 1.980 & 0.012 & 1.556 & 1.712 & 1.985 \\
$\mathcal{C} v \mathcal{M}$ & 256 & 1.830 & $<0.002$ & 1.224 & 1.329 & 1.550 \\
$\mathcal{C} v \mathcal{M}$ & 512 & 1.368 & 0.008 & 0.986 & 1.145 & 1.332 \\
$\mathcal{K S}$ & 64 & 23.547 & $<0.002$ & 7.505 & 8.966 & 12.824 \\
$\mathcal{K} \mathcal{S}$ & 128 & 20.775 & $<0.002$ & 11.276 & 14.389 & 18.939 \\
$\mathcal{K S}$ & 256 & 19.390 & $<0.002$ & 6.390 & 7.482 & 10.234 \\
$\mathcal{K} \mathcal{S}$ & 512 & 14.781 & $<0.002$ & 6.651 & 8.557 & 10.397 \\
\hline
\end{tabular}




\section{APPENDIX II: PROOF OF MAIN RESULTS}

We introduce some notation. In what follows, we denote

$$
\begin{aligned}
\stackrel{\circ}{I}_{x, b}(j) & =\frac{I_{x, b}(j)}{\left|\mathcal{B}\left(\frac{n(b-1)}{T} ; j\right)\right|^{2}} ; \quad \bar{\circ}_{x}(j)=\frac{1}{\mathbf{B}} \sum_{b=1}^{\mathbf{B}} \stackrel{\circ}{I}_{x, b}(j) \\
R_{n, b}(j) & =\stackrel{\circ}{I}_{x, b}(j)-I_{\varepsilon, b}(j) ; \bar{R}_{n}(j)=\frac{1}{\mathbf{B}} \sum_{b=1}^{\mathbf{B}} R_{n, b}(j) \\
\stackrel{\circ}{I}_{\varepsilon, b}(j) & =I_{\varepsilon, b}(j)-1 ; \bar{\circ}_{\varepsilon}(j)=\frac{1}{\mathbf{B}} \sum_{b=1}^{\mathbf{B}} \stackrel{\circ}{I}_{\varepsilon, b}(j) \\
\breve{R}_{n, b}(j) & =R_{n, b}(j)-\mathcal{E}\left(R_{n, b}(j)\right) ; \quad \overline{\breve{R}}_{n}(j)=\frac{1}{\mathbf{B}} \sum_{b=1}^{\mathbf{B}} \breve{R}_{n, b}(j) .
\end{aligned}
$$

Observe that under $H_{0}$, we have that $\stackrel{\circ}{I}_{x, b}(j)=f^{-1}(j) I_{x, b}(j)$. In addition, for notational simplicity, we assume that $\sigma_{\varepsilon}^{2}=1$ without loss of generality.

We also introduce the following definition: We say that a process $\mathrm{X}_{n, \mathbf{B}}\left(\frac{\mathbf{b}^{*}}{\mathbf{B}} ; \frac{\mathbf{j}^{*}}{\tilde{n}}\right)$ satisfies Condition $B W$ if

$$
\sup _{\mathbf{j}^{*}=1, \ldots, \tilde{n} ; \mathbf{b}^{*}=1, \ldots, \mathbf{B}}\left|\mathrm{X}_{n, \mathbf{B}}\left(\frac{\mathbf{b}^{*}}{\mathbf{B}} ; \frac{\mathbf{j}^{*}}{\tilde{n}}\right)\right|=o_{p}\left(T^{-1 / 2}\right) \text {. }
$$

Recall that according to Bickel and Wichura (1972), a sufficient condition for $B W$ is that for some $\alpha \geq 1$ and $\delta>0$,

$$
\begin{gathered}
\mathcal{E}\left|\mathrm{X}_{n, \mathbf{B}}\left(\frac{\mathbf{b}_{2}^{*}}{\mathbf{B}} ; \frac{\mathbf{j}_{2}^{*}}{\tilde{n}}\right)-\mathrm{X}_{n, \mathbf{B}}\left(\frac{\mathbf{b}_{1}^{*}}{\mathbf{B}} ; \frac{\mathbf{j}_{2}^{*}}{\tilde{n}}\right)-\mathrm{X}_{n, \mathbf{B}}\left(\frac{\mathbf{b}_{2}^{*}}{\mathbf{B}} ; \frac{\mathbf{j}_{1}^{*}}{\tilde{n}}\right)+\mathrm{X}_{n, \mathbf{B}}\left(\frac{\mathbf{b}_{1}^{*}}{\mathbf{B}} ; \frac{\mathbf{j}_{1}^{*}}{\tilde{n}}\right)\right|^{\alpha} \\
=o\left(\frac{1}{T^{\alpha / 2}}\left(\frac{\mathbf{j}_{2}^{*}-\mathbf{j}_{1}^{*}}{\tilde{n}}\right)^{1+\delta}\left(\frac{\mathbf{b}_{2}^{*}-\mathbf{b}_{1}^{*}}{\mathbf{B}}\right)^{1+\delta}\right) .
\end{gathered}
$$

Finally, recall that $\breve{\mathcal{T}}_{n, \mathbf{B}}\left(\frac{\mathbf{b}^{*}}{\mathbf{B}} ; \frac{\mathbf{j}^{*}}{\tilde{n}}\right) \equiv \mathcal{T}_{n, \mathbf{B}}\left(\frac{\mathbf{b}^{*}}{\mathbf{B}} ; \frac{\mathbf{j}^{*}}{\tilde{n}}\right)$ under $H_{0}$

\subsection{Proof of Proposition 1.}

We begin by demonstrating the statistical properties of $\widehat{\sigma}_{\varepsilon}^{2}$. Recall that is $\widehat{\sigma}_{\varepsilon}^{2}=\mathbf{B}^{-1} \sum_{b=1}^{\mathbf{B}} \widehat{\sigma}_{\varepsilon}^{2}(b)$, where $\widehat{\sigma}_{\varepsilon}^{2}(b)$ is given in $(2.8)$. Now, we have that

$$
\widehat{\sigma}_{\varepsilon}^{2}(b)-1=: \frac{1}{n} \sum_{t=1}^{n}\left(\varepsilon_{t+(b-1) n}^{2}-1\right)+\left(\widehat{\sigma}_{\varepsilon}^{2}(b)-\widetilde{\sigma}_{\varepsilon}^{2}(b)\right),
$$

recall that we assumed $\sigma_{\varepsilon}^{2}=1$ for notational simplicity, where

$$
\tilde{\sigma}_{\varepsilon}^{2}(b)=\frac{1}{n} \sum_{t=1}^{n} \varepsilon_{t+(b-1) n}^{2} ; \quad b=1, \ldots, \mathbf{B} .
$$

From here and Lemma 7 , it is obvious that $\widehat{\sigma}_{\varepsilon}^{2}-\sigma_{\varepsilon}^{2}=o_{p}(1)$. Further,

$$
\frac{1}{T} \sum_{t=1}^{T} \widehat{\varepsilon}_{t}^{4}=\frac{1}{\mathbf{B}} \sum_{b=1}^{\mathbf{B}} \frac{1}{n} \sum_{t=1}^{n}\left(\widehat{\varepsilon}_{t+(b-1) n}^{4}-\varepsilon_{t+(b-1) n}^{4}\right)+\frac{1}{\mathbf{B}} \sum_{b=1}^{\mathbf{B}} \frac{1}{n} \sum_{t=1}^{n} \varepsilon_{t+(b-1) n}^{4} .
$$

Now, the first term on the right side of the last displayed expression converges to zero in probability by Lemma 8, whereas by Condition $C 1$ and the weak law of large numbers, the second term converges to $3 \sigma_{\varepsilon}^{4}+\kappa_{4}$ in probability. Now, standard arguments conclude that $\widehat{\kappa}_{4}-\kappa_{4}=o_{p}(1)$.

\subsection{Proof of Proposition 2.}


Using Taylor's expansion around 1 of $\bar{I}_{x}(j)^{-1}$, we obtain the following decomposition for $\breve{\mathcal{T}}_{n, \mathbf{B}}\left(\frac{\mathbf{b}^{*}}{\mathbf{B}} ; \frac{\mathbf{j}^{*}}{\tilde{n}}\right)$,

$$
\begin{aligned}
& \frac{1}{\tilde{n}} \sum_{j=1}^{\mathbf{j}^{*}}\left\{\frac{1}{\mathbf{B}} \sum_{b=1}^{\mathbf{b}^{*}}\left\{\stackrel{\circ}{I}_{x, b}(j)-\overline{\bar{I}}_{x}(j)\right\} \sum_{k=0}^{2} \frac{(-1)^{k}}{k !}\left(\overline{\bar{I}}_{x}(j)-1\right)^{k}\right\} \\
& +\frac{1}{\tilde{n}} \sum_{j=1}^{\mathbf{j}^{*}}\left\{\frac{1}{\mathbf{B}} \sum_{b=1}^{\mathbf{b}^{*}}\left\{\stackrel{\circ}{I}_{x, b}(j)-\overline{\check{I}}_{x}(j)\right\} \frac{\left(\bar{I}_{x}(j)-1\right)^{3}}{(1-\alpha)+\alpha \bar{I}_{x}(j)}\right\},
\end{aligned}
$$

where $\alpha=: \alpha(j) \in(0,1)$. Notice that Lemma 4 and the well-known inequalities $\sup _{j}\left|\bar{I}_{\varepsilon}(j)\right|=$ $O_{p}\left(n^{1 / 4} \mathbf{B}^{-1 / 2}\right)$, and thus, by $C 2$, this implies that $\left(\inf _{j}\left|\bar{I}_{x}(j)\right|\right)^{-1}<C$ for some finite positive constant $C$; hence,

$$
\sup _{j}\left((1-\alpha)+\alpha \overline{\bar{I}}_{x}(j)\right)^{-1}=O_{p}(1) .
$$

We first examine the second term of (5.5) and in particular

$$
\begin{aligned}
& \frac{1}{\tilde{n}} \sum_{j=1}^{\mathbf{j}^{*}} \frac{1}{\mathbf{B}} \sum_{b=1}^{\mathbf{b}^{*}}\left\{\stackrel{\circ}{I}_{x, b}(j)-1\right\} \frac{\left(\overline{\bar{I}}_{x}(j)-1\right)^{3}}{(1-\alpha)+\alpha \overline{\bar{I}}_{x}(j)} \\
= & \frac{1}{\tilde{n}} \sum_{j=1}^{\mathbf{j}^{*}} \frac{1}{\mathbf{B}}\left(\sum_{b=1}^{\mathbf{b}^{*}} R_{n, b}(j)+\sum_{b=1}^{\mathbf{b}^{*}} \stackrel{\circ}{I}_{\varepsilon, b}(j)\right) \frac{\left(\overline{\check{I}}_{x}(j)-1\right)^{3}}{(1-\alpha)+\alpha \bar{I}_{x}(j)} .
\end{aligned}
$$

Now, because (5.6), we have that the contribution due to $\sum_{b=1}^{\mathbf{b}^{*}} R_{n, b}(j)$ on the right side of (5.7) is

$$
\begin{aligned}
& \left|\frac{1}{\tilde{n}} \sum_{j=1}^{\mathbf{j}^{*}}\left(\frac{1}{\mathbf{B}} \sum_{b=1}^{\mathbf{b}^{*}} R_{n, b}(j)\right) \frac{\left(\bar{R}_{n}(j)\right)^{3}}{(1-\alpha)+\alpha \bar{I}_{x}(j)}\right| \\
= & O_{p}(1)\left(\frac{1}{\tilde{n}} \sum_{j=1}^{\mathbf{j}^{*}}\left|\frac{1}{\mathbf{B}} \sum_{b=1}^{\mathbf{b}^{*}} R_{n, b}(j)\right|\left|\bar{R}_{n}(j)\right|^{3}\right)
\end{aligned}
$$

and hence Lemma 4 and Condition $C 2$ imply that the second factor on the right side of the last displayed equality satisfies (5.2) with $\alpha=1$ there and, hence, Condition $B W$. Similarly, the contribution due to $\sum_{b=1}^{\mathbf{b}^{*}} \stackrel{\circ}{I}, b(j)$ on the right side of $(5.7)$ is

$$
O_{p}(1)\left(\frac{1}{\tilde{n}} \sum_{j=1}^{\mathbf{j}^{*}}\left|\frac{1}{\mathbf{B}} \sum_{b=1}^{\mathbf{b}^{*}} \stackrel{\circ}{I}, b_{\varepsilon, 1}(j)\right|\left|\overline{\bar{I}}_{\varepsilon, b}(j)\right|^{3}\right)
$$

which satisfies (5.2) and, hence, Condition $B W$ because

$$
\begin{aligned}
& \frac{1}{\tilde{n}} \sum_{j=\mathbf{j}_{1}^{*}+1}^{\mathbf{j}_{2}^{*}}\left|\frac{1}{\mathbf{B}} \sum_{b=\mathbf{b}_{1}^{*}+1}^{\mathbf{b}_{2}^{*}} \stackrel{\circ}{I}_{\varepsilon, b}(j)\right|\left|\bar{I}_{\varepsilon, b}(j)\right|^{3} \\
\leq & 4 \frac{1}{\tilde{n}} \sum_{j=\mathbf{j}_{1}^{*}+1}^{\mathbf{j}_{2}^{*}}\left|\frac{1}{\mathbf{B}} \sum_{b=\mathbf{b}_{1}^{*}+1}^{\mathbf{b}_{2}^{*}} \stackrel{\circ}{I}_{\varepsilon, b}(j)\right|^{4}+4 \frac{1}{\tilde{n}} \sum_{j=\mathbf{j}_{1}^{*}+1}^{\mathbf{j}_{2}^{*}}\left|\frac{1}{\mathbf{B}} \sum_{b=\mathbf{b}_{1}^{*}+1}^{\mathbf{b}_{2}^{*}} \stackrel{\circ}{I}_{\varepsilon, b}(j)\right|\left|\frac{1}{\mathbf{B}} \sum_{b \neq \mathbf{b}_{1}^{*}+1}^{\mathbf{b}_{2}^{*}} \stackrel{\circ}{I}_{\varepsilon, b}(j)\right|^{3} .
\end{aligned}
$$

From here, the proof is standard after observing that $\stackrel{\circ}{\varepsilon, b}_{\varepsilon}(j)$ and $\stackrel{\circ}{\varepsilon, v}_{\varepsilon}(j)$ are independent if $b \neq v$, and $C 2$ implies that $\mathbf{B}^{-2}=o\left(\mathbf{B}^{-1 / 2} T^{-1 / 2}\right)$. 
Next, the first term of (5.5), which is

$$
\begin{aligned}
& \frac{1}{\tilde{n}} \sum_{j=1}^{\mathbf{j}^{*}} \frac{1}{\mathbf{B}} \sum_{b=1}^{\mathbf{b}^{*}}\left\{\breve{R}_{n, b}(j)-\overline{\breve{R}}_{n}(j)\right\} \sum_{k=0}^{2} \frac{(-1)^{k}}{k !}\left(\overline{\check{I}}_{x}(j)-1\right)^{k} \\
& +\frac{1}{\tilde{n}} \sum_{j=1}^{\mathbf{j}^{*}} \frac{1}{\mathbf{B}} \sum_{b=1}^{\mathbf{b}^{*}}\left\{\stackrel{\circ}{I}_{\varepsilon, b}(j)-\overline{\bar{I}}_{\varepsilon}(j)\right\} \sum_{k=1}^{2} \frac{(-1)^{k}}{k !}\left(\overline{\check{I}}_{x}(j)-1\right)^{k} \\
& +\frac{1}{\tilde{n}} \sum_{j=1}^{\mathbf{j}^{*}} \frac{1}{\mathbf{B}} \sum_{b=1}^{\mathbf{b}^{*}}\left\{\stackrel{\circ}{I}_{\varepsilon, b}(j)-\overline{\bar{I}}_{\varepsilon}(j)\right\}
\end{aligned}
$$

since $\mathcal{E}\left(\stackrel{\circ}{I}_{x}(j)\right)=\mathcal{E}\left(\stackrel{\circ}{I}_{x, b}(j)\right)$ and $\stackrel{\circ}{I}_{x, b}(j)-\mathcal{E}\left(\stackrel{\circ}{I}_{x, b}(j)\right)=\breve{R}_{n, b}(j)+\stackrel{\circ}{I}_{\varepsilon, b}(j)$.

We first show the first term of (5.8) satisfies Condition $B W$. Indeed, by Lemmas 4 and 5 , we have that the contribution due to $\sum_{k=1}^{2} \breve{R}_{n}^{k}(j)$ in the term satisfies Condition $B W$. Thus, noticing that $\overline{\bar{I}}_{x}(j)-1=\overline{\breve{R}}_{n}(j)+\mathcal{E}\left(R_{n, b}(j)\right)+\bar{I}_{\varepsilon}(j)$, it suffices to show that

$$
\frac{1}{\tilde{n}} \sum_{j=\mathbf{j}_{1}^{*}+1}^{\mathbf{j}_{2}^{*}}\left(\frac{1}{\mathbf{B}} \sum_{b=\mathbf{b}_{1}^{*}+1}^{\mathbf{b}_{2}^{*}} \breve{R}_{n, b}(j)\right) \sum_{k=0}^{2} \frac{(-1)^{k}}{k !} \stackrel{\bar{\rho}}{I}_{\varepsilon}(j)
$$

satisfies (5.2). When $k=0$, this is a direct consequence of Lemma 5 . Next, because $C 1^{\prime}$ implies that $\mathcal{E}\left(\overline{\bar{o}}_{\varepsilon}(j)^{2 k}\right)=O\left(\mathbf{B}^{-k}\right)$, the Cauchy-Schwarz inequality, and then Lemma 5 , indicates that the contribution of the first absolute moment of the terms due to $k=2$ in (5.9) is bounded by

$$
\frac{1}{\mathbf{B} \tilde{n}} \sum_{j=\mathbf{j}_{1}^{*}+1}^{\mathbf{j}_{2}^{*}}\left(\mathcal{E}\left(\frac{1}{\mathbf{B}} \sum_{b=\mathbf{b}_{1}^{*}+1}^{\mathbf{b}_{2}^{*}} \breve{R}_{n, b}(j)\right)^{2}\right)^{1 / 2}=\frac{C}{T^{1 / 2} \mathbf{B}}\left(\frac{\mathbf{j}_{2}^{*}-\mathbf{j}_{1}^{*}}{\tilde{n}}\right)\left(\frac{\mathbf{b}_{2}^{*}-\mathbf{b}_{1}^{*}}{\mathbf{B}}\right)^{1 / 2} .
$$

Thus, it satisfies (5.2) because $C 2$ implies that for some $\delta>0, \mathbf{B}^{-1 / 2} \leq T^{-\delta} \tilde{n}^{-\delta}$.

To finish demonstrating that the first term of (5.8) satisfies Condition $B W$, it remains to do so for (5.9) when $k=1$, that is,

$$
\frac{1}{\tilde{n}} \sum_{j=\mathbf{j}_{1}^{*}+1}^{\mathbf{j}_{2}^{*}}\left(\frac{1}{\mathbf{B}} \sum_{b=\mathbf{b}_{1}^{*}+1}^{\mathbf{b}_{2}^{*}} \breve{R}_{n, b}(j)\right) \overline{\bar{I}}_{\varepsilon}(j) .
$$

To that end, using (5.29) but with (2.27), (2.28) and (2.29) instead of (2.10) and (2.11) there, it suffices to examine that

$$
\begin{aligned}
& \frac{1}{\tilde{n}} \sum_{j=\mathbf{j}_{1}^{*}+1}^{\mathbf{j}_{2}^{*}} \overline{\check{I}}_{\varepsilon}(j) \frac{1}{\mathbf{B}} \sum_{b=\mathbf{b}_{1}^{*}+1}^{\mathbf{b}_{2}^{*}}\left(\left|\dot{\mathrm{Y}}_{n, b}(j ; 0)\right|^{2}-\mathcal{E}\left|\dot{\mathrm{Y}}_{n, b}(j ; 0)\right|^{2}\right) \\
& \frac{1}{\tilde{n}} \sum_{j=\mathbf{j}_{1}^{*}+1}^{\mathbf{j}_{2}^{*}} \overline{\check{I}}_{\varepsilon}(j) \overline{\tilde{I}}_{\varepsilon}\left(j, n^{1 / 2}\right)+\frac{1}{\tilde{n}} \sum_{j=\mathbf{j}_{1}^{*}+1}^{\mathbf{j}_{2}^{*}} \overline{\check{I}}_{\varepsilon}(j)\left(\overline{\tilde{I}}_{\varepsilon}(j, 0)-\overline{\tilde{I}}_{\varepsilon}\left(j, n^{1 / 2}\right)\right),
\end{aligned}
$$

satisfy Condition $B W$, where $\dot{\mathrm{Y}}_{n, b}(j ; 0)=\check{\mathrm{Y}}_{n, b}(j ; 0)+\ddot{\mathrm{Y}}_{n, b}(j)$ and

$$
\overline{\tilde{I}}_{\varepsilon}(j ; q)=\frac{1}{\mathbf{B}} \sum_{b=\mathbf{b}_{1}^{*}+1}^{\mathbf{b}_{2}^{*}}\left(\mathcal{J}_{\varepsilon, b}(j) \dot{\mathrm{Y}}_{n, b}(-j ; q)-\mathcal{E}\left(\mathcal{J}_{\varepsilon, b}(j) \dot{\mathrm{Y}}_{n, b}(-j ; q)\right)\right) .
$$


Because

$$
\begin{aligned}
\left|\dot{\beta}_{t+b n, T}(\ell)\right| & =\left|\beta_{t+b n, T}(\ell)-\beta\left(\frac{n b}{T} ; \ell\right)\right|=O\left(T^{-1}|v(\ell)|\right) \\
\left|\ddot{\beta}\left(\frac{t+n b}{T} ; \ell\right)\right| & =\left|\beta\left(\frac{t+n b}{T} ; \ell\right)-\beta\left(\frac{n b}{T} ; \ell\right)\right| \leq C v(\ell) / n^{-1 / 2}
\end{aligned}
$$

it standard to conclude that the contribution to (5.10) or (5.11) of $\ddot{Y}_{n, b}(j)$ satisfies the sufficient condition (5.2). Thus, it suffices to examine the behavior of (5.10) or (5.11) with $\dot{\mathrm{Y}}_{n, b}(j ; 0)$ replaced with $\check{Y}_{n, b}(j ; 0)$. Now, standard inequalities yield that the first absolute moment of (5.10) is bounded by

$$
\begin{aligned}
& \frac{1}{\tilde{n}} \sum_{j=\mathbf{j}_{1}^{*}+1}^{\mathbf{j}_{2}^{*}}\left(\left.\mathcal{E}\left(\overline{-}_{\varepsilon}^{2}(j)\right) \mathcal{E}\left|\frac{1}{\mathbf{B}} \sum_{b=\mathbf{b}_{1}^{*}+1}^{\mathbf{b}_{2}^{*}}\right| \check{\mathrm{Y}}_{n, b}(j ; 0)\right|^{2}-\left.\mathcal{E}\left|\check{\mathrm{Y}}_{n, b}(j ; 0)\right|^{2}\right|^{2}\right)^{1 / 2} \\
= & O\left(\frac{1}{\mathbf{B}^{1 / 2} \tilde{n}}\left(\frac{\mathbf{j}_{2}^{*}-\mathbf{j}_{1}^{*}}{\tilde{n}}\right)\left(\frac{\mathbf{b}_{2}^{*}-\mathbf{b}_{1}^{*}}{\mathbf{B}}\right)\right),
\end{aligned}
$$

see (5.34) and (5.35) in Lemma 4. Therefore, Conditions $C 2^{\prime}$ and $C 2$ imply that (5.10) satisfies Condition $B W$.

Next, (5.11), with $\dot{\mathrm{Y}}_{n, b}(j ; 0)$ replaced by $\check{\mathrm{Y}}_{n, b}(j ; 0)$. The second moment of the first term is

$$
\frac{1}{\tilde{n}^{2}} \sum_{j, k=\mathbf{j}_{1}^{*}+1}^{\mathbf{j}_{2}^{*}} \mathcal{E}\left(\overline{\check{I}}_{\varepsilon}(j) \overline{\check{I}}_{\varepsilon}(k) \overline{\tilde{I}}_{\varepsilon}\left(j, n^{1 / 2}\right) \overline{\tilde{I}}_{\varepsilon}\left(k, n^{1 / 2}\right)\right)=o\left(\frac{1}{T}\left(\frac{\mathbf{j}_{2}^{*}-\mathbf{j}_{1}^{*}}{\tilde{n}}\right)^{2}\left(\frac{\mathbf{b}_{2}^{*}-\mathbf{b}_{1}^{*}}{\mathbf{B}}\right)^{2}\right)
$$

using that $\sum_{\ell=n^{1 / 2}}^{\infty}|\beta(u ; \ell)|=o\left(n^{-1 / 2}\right)$ by $C 1^{\prime}$ and the independence of the sequence $\left\{\varepsilon_{t}\right\}_{t \in \mathbb{Z}}$. Thus, the first term of (5.11) satisfies Condition $B W$. Finally, consider the second term of (5.11). Using the definition of $\check{Y}_{n, b}(j ; a)$ in $(2.28)$, it suffices to consider

$$
\frac{1}{\tilde{n}} \sum_{j=1}^{\mathbf{j}^{*}} \overline{\bar{I}}_{\varepsilon}(j) \mathcal{B}\left(u ; e^{-i \lambda_{j}}\right) \frac{1}{\mathbf{B}} \sum_{b=\mathbf{b}_{1}^{*}+1}^{\mathbf{b}_{2}^{*}}\left(\mathcal{J}_{\varepsilon, b}(j) \dot{\mathrm{Y}}_{n, b}\left(-j ; n^{1 / 2}\right)-\mathcal{E} \mathcal{J} \varepsilon, b(j) \dot{\mathbf{Y}}_{n, b}\left(-j ; n^{1 / 2}\right)\right)
$$

where $\dot{Y}_{n, b}\left(-j ; n^{1 / 2}\right)=n^{-1 / 2} \sum_{\ell=1}^{n^{1 / 2}} \ell^{1 / 2} \beta(u ; \ell) e^{-i \ell \lambda_{j}}\left(\ell^{-1 / 2} \sum_{t=n-\ell}^{n} \varepsilon_{t+(b-1) n} e^{-i t \lambda_{j}}\right)$. However, $\mathcal{E}\left|\dot{\mathrm{Y}}_{n, b}\left(-j ; n^{1 / 2}\right)\right|^{2}=o\left(n^{-1}\right)$, and thus, the second moment of the last displayed expression is $O\left(\left(\frac{\mathbf{j}_{2}^{*}-\mathbf{j}_{1}^{*}}{n}\right)^{2}\left(\frac{\mathbf{b}_{2}^{*}-\mathbf{b}_{1}^{*}}{\mathbf{B}}\right) / \mathbf{B}^{2} n\right)$, and hence, it satisfies Condition $B W$ by Condition $C 2$. This completes the proof that the first term of (5.8) satisfies Condition $B W$.

Next, the second term of (5.8), i.e.,

$$
\frac{1}{\tilde{n}} \sum_{j=1}^{\mathbf{j}^{*}} \frac{1}{\mathbf{B}} \sum_{b=1}^{\mathbf{b}^{*}}\left\{\stackrel{\circ}{I}_{\varepsilon, b}(j)-\overline{\stackrel{\circ}{I}}_{\varepsilon}(j)\right\} \sum_{k=1}^{2} \frac{(-1)^{k}}{k !}\left(\breve{\breve{R}}_{n}(j)+\mathcal{E}\left(R_{n, b}(j)\right)+\overline{\check{\circ}}_{\varepsilon}(j)\right)^{k} .
$$

Proceeding as with the first term of (5.8), the contribution due to $\breve{R}_{n}(j)+\mathcal{E}\left(R_{n, b}(j)\right)$ satisfies Condition $B W$. Thus, we only need to examine

$$
\frac{1}{\tilde{n}} \sum_{j=1}^{\mathbf{j}^{*}}\left(\frac{1}{\mathbf{B}} \sum_{b=1}^{\mathbf{b}^{*}} \stackrel{\circ}{I}_{\varepsilon, b}(j)-\frac{\mathbf{b}^{*}}{\mathbf{B}} \overline{\check{I}}_{\varepsilon}(j)\right) \sum_{k=1}^{2} \frac{(-1)^{k} \bar{o}_{\bar{I}}^{k}}{k !}(j) .
$$


The contribution due to $k=2$ is $o_{p}\left(T^{-1 / 2}\right)$ uniformly in $\mathbf{j}^{*}$ and $\mathbf{b}^{*}$ because $\sup _{\mathbf{j}^{*}}\left|\sum_{j=1}^{\mathbf{j}^{*}} a_{j}\right| \leq \sum_{j=1}^{\tilde{n}}\left|a_{j}\right|$, $\mathcal{E}\left(\sum_{b=\mathbf{b}_{1}^{*}+1}^{\mathbf{b}_{2}^{*}} \stackrel{\circ}{\varepsilon}, b_{(j)}\right)^{2 k}=O\left(\left(\mathbf{b}_{2}^{*}-\mathbf{b}_{1}^{*}\right)^{k}\right)$, and then Condition $C 2$ implies that

$$
\begin{aligned}
& \mathcal{E}\left(\sup _{\mathbf{j}^{*}} \frac{1}{\tilde{n}}\left|\sum_{j=1}^{\mathbf{j}^{*}} \frac{1}{\mathbf{B}} \sum_{b=\mathbf{b}_{1}^{*}+1}^{\mathbf{b}_{2}^{*}} \stackrel{\circ}{I}_{\varepsilon, b}(j) \overline{\bar{I}}_{\varepsilon}^{2}(j)\right|\right) \\
\leq & \frac{1}{\tilde{n}} \sum_{j=1}^{\tilde{n}} \mathcal{E}\left(\left|\frac{1}{\mathbf{B}} \sum_{b=\mathbf{b}_{1}^{*}+1}^{\mathbf{b}_{2}^{*}} \stackrel{\circ}{I}, b_{\varepsilon}(j) \overline{\bar{I}}_{\varepsilon}^{2}(j)\right|\right)=o\left(\frac{1}{T^{1 / 2}}\left(\frac{\mathbf{b}_{2}^{*}-\mathbf{b}_{1}^{*}}{\mathbf{B}}\right)^{1+\delta}\right) .
\end{aligned}
$$

Next, the contribution due to $k=1$ in (5.13), which is

$$
\begin{aligned}
& \frac{1}{\tilde{n}} \sum_{j=1}^{\mathbf{j}^{*}} \frac{1}{\mathbf{B}}\left(\sum_{b=1}^{\mathbf{b}^{*}} \stackrel{\circ}{I}_{\varepsilon, b}(j) \overline{\check{I}}_{\varepsilon}(j)-\frac{\mathbf{b}^{*}}{\mathbf{B}}\left(1+\frac{\kappa_{4}}{n}\right)\right) \\
& -\frac{\mathbf{b}^{*}}{\mathbf{B}} \frac{1}{\tilde{n}} \sum_{j=1}^{\mathbf{j}^{*}}\left\{\overline{\bar{\rho}}^{2}(j)-\frac{1}{\mathbf{B}}\left(1+\frac{\kappa_{4}}{n}\right)\right\}
\end{aligned}
$$

after we realize that $\mathcal{E}\left(\sum_{b=1}^{\mathbf{b}^{*}} \stackrel{\circ}{I}, b_{\varepsilon}(j) \overline{\bar{I}}_{\varepsilon}(j)\right)=\mathbf{b}^{*} \mathcal{E}\left(\bar{I}_{\mathcal{I}}^{2}(j)\right)=\left(1+\frac{\kappa_{4}}{n}\right) \mathbf{b}^{*} / \mathbf{B}$. Thus, it suffices to examine the behavior of

$$
\mathcal{E}\left(\frac{1}{\tilde{n}} \sum_{j=\mathbf{j}_{1}^{*}+1}^{\mathbf{j}_{2}^{*}}\left\{\frac{1}{\mathbf{B}} \sum_{b=\mathbf{b}_{1}^{*}+1}^{\mathbf{b}_{2}^{*}} i_{\varepsilon, b}(j) \bar{I}_{\varepsilon}(j)-\frac{\left(\mathbf{b}_{2}^{*}-\mathbf{b}_{1}^{*}\right)}{\mathbf{B}^{2}}\left(1+\frac{\kappa_{4}}{n}\right)\right\}\right)^{2}
$$

which is

$$
\begin{aligned}
& \quad \frac{1}{\tilde{n}^{2}}\left\{\sum_{j, k=\mathbf{j}_{1}^{*}+1}^{\mathbf{j}_{2}^{*}} \mathcal{E}\left(\frac{1}{\mathbf{B}} \sum_{b=\mathbf{b}_{1}^{*}+1}^{\mathbf{b}_{2}^{*}} \stackrel{\circ}{I}_{\varepsilon, b}(j) \overline{\bar{I}}_{\varepsilon}(k)\right) \mathcal{E}\left(\frac{1}{\mathbf{B}} \sum_{b=\mathbf{b}_{1}^{*}+1}^{\mathbf{b}_{2}^{*}} \stackrel{\circ}{I}_{\varepsilon, b}(k) \overline{\check{I}}_{\varepsilon}(j)\right)\right. \\
& +\sum_{j, k=\mathbf{j}_{1}^{*}+1}^{\mathbf{j}_{2}^{*}} \mathcal{E}\left(\frac{1}{\mathbf{B}} \sum_{b=\mathbf{b}_{1}^{*}+1}^{\mathbf{b}_{2}^{*}} \stackrel{\circ}{I}_{\varepsilon, b}(j) \frac{1}{\mathbf{B}} \sum_{b=\mathbf{b}_{1}^{*}+1}^{\mathbf{b}_{2}^{*}} \stackrel{\circ}{I}_{\varepsilon, b}(k)\right) \mathcal{E}\left(\overline{\bar{I}}_{\varepsilon}(j) \overline{\stackrel{I}{I}}_{\varepsilon}(k)\right) \\
& \left.+\sum_{j, k=\mathbf{j}_{1}^{*}+1}^{\mathbf{j}_{2}^{*}} \frac{1}{\mathbf{B}^{2}} \sum_{b, v=\mathbf{b}_{1}^{*}+1}^{\mathbf{b}_{2}^{*}} C u m\left(\stackrel{\circ}{I}_{\varepsilon, b}(j) ; \stackrel{\circ}{I}, v_{\varepsilon, v}(k) ; \overline{\check{I}}_{\varepsilon}(j) ; \bar{I}_{\varepsilon}(k)\right)\right\} \\
& =O\left(\left(\mathbf{j}_{2}^{*}-\mathbf{j}_{1}^{*}\right)\left(\mathbf{b}_{2}^{*}-\mathbf{b}_{1}^{*}\right) / \mathbf{B}^{3} n^{2}\right)
\end{aligned}
$$

using Brillinger's (1980) Theorems 2.3.2 and 4.3.1., and in particular expressions in (2.3.7) and (4.3.15)), as $(5.55)$ and

$$
\sum_{j, k=\mathbf{j}_{1}^{*}+1}^{\mathbf{j}_{2}^{*}} \sum_{b=\mathbf{b}_{1}^{*}+1}^{\mathbf{b}_{2}^{*}} \operatorname{Cum}\left(I_{\varepsilon, b}(j) ; I_{\varepsilon, b}(j) ; I_{\varepsilon, b}(k) ; I_{\varepsilon, b}(k)\right)=O\left(\left(\mathbf{b}_{2}^{*}-\mathbf{b}_{1}^{*}\right)\left(\mathbf{j}_{2}^{*}-\mathbf{j}_{1}^{*}\right)\right) .
$$

Recall that $C 1^{\prime}$ implies that ${\stackrel{\circ}{\varepsilon}, b_{1}}_{1}(j)$ and ${\stackrel{\circ}{\varepsilon}, b_{2}}_{2}(k)$ are independent for all $j, k$ if $b_{1} \neq b_{2}$.

Therefore, we conclude that uniformly in $\mathbf{j}^{*}$ and $\mathbf{b}^{*}$, the first and second terms of (5.8) satisfy Condition $B W$, and hence, (5.5) is

$$
\frac{1}{\tilde{n}} \sum_{j=1}^{\mathbf{j}^{*}} \frac{1}{\mathbf{B}} \sum_{p=1}^{\mathbf{b}^{*}}\left\{\stackrel{\circ}{I}_{\varepsilon, b}(j)-\overline{\bar{I}}_{\varepsilon}(j)\right\}+o_{p}\left(\frac{1}{T^{1 / 2}}\right)
$$


as we showed above that the second term of (5.5) satisfied (5.2). Thus, the proof is completed if we show that the first term of the last displayed expression

$$
\frac{1}{(\tilde{n} \mathbf{B})^{1 / 2}}\left\{\sum_{j=1}^{\mathbf{j}^{*}} \sum_{b=1}^{\mathbf{b}^{*}} \stackrel{\circ}{I}_{\varepsilon, b}(j)-\left(\frac{\mathbf{b}^{*}}{\mathbf{B}}\right) \sum_{j=1}^{\mathbf{j}^{*}} \sum_{b=1}^{\mathbf{B}} \stackrel{\circ}{I}_{\varepsilon, b}(j)\right\} \Rightarrow \mathcal{B S}\left([0,1]^{2}\right) .
$$

To that end, it is standard to show that

$$
\frac{1}{\tilde{n} \mathbf{B}} \mathcal{E}\left(\sum_{j=1}^{\mathbf{j}_{1}^{*}} \sum_{b=1}^{\mathbf{b}_{1}^{*}} \stackrel{\circ}{I}_{\varepsilon, b}(j) \sum_{k=1}^{\mathbf{j}_{2}^{*}} \sum_{v=1}^{\mathbf{b}_{2}^{*}} \stackrel{\circ}{I}_{\varepsilon, v}(k)\right)=\frac{\mathbf{b}_{1}^{*}}{\mathbf{B}}\left[\left(\frac{\mathbf{j}_{1}^{*}}{\tilde{n}}\right)+\left(\frac{\mathbf{j}_{1}^{*}}{\tilde{n}}\right)\left(\frac{\mathbf{j}_{2}^{*}}{\tilde{n}}\right) \kappa_{4}\right],
$$

where we have assumed without loss of generality that $\mathbf{j}_{1}^{*} \leq \mathbf{j}_{2}^{*}$ and $\mathbf{b}_{1}^{*} \leq \mathbf{b}_{2}^{*}$ and the independence of $\stackrel{\circ}{I}_{\varepsilon, b}(j)$ and $\stackrel{\circ}{I}_{\varepsilon, v}(j)$ for $b \neq v$ by Condition $C 1^{\prime}$. Thus, the covariance structure of (5.14) is, after standard algebra, given by $(2.30)$.

From here, the proof concludes by standard arguments if we show that

$$
\frac{1}{(\tilde{n} \mathbf{B})^{1 / 2}} \sum_{j=1}^{\mathbf{j}^{*}} \sum_{b=1}^{\mathbf{b}^{*}} \stackrel{\circ}{I}_{\varepsilon, b}(j)=\frac{1}{(\tilde{n} \mathbf{B})^{1 / 2}} \sum_{b=1}^{\mathbf{b}^{*}} \sum_{j=1}^{\mathbf{j}^{*}} \stackrel{\circ}{I}_{\varepsilon, b}(j)
$$

converges in distribution to a normal random variable. However, this is the case, as $\tilde{n}^{-1 / 2} \sum_{j=1}^{\mathbf{j}^{*}} \stackrel{\circ}{\varepsilon}_{\varepsilon, b}(j)$ is a triangular array of independent identically distributed random variables with finite second moments. This completes the proof.

\subsection{Proof of Theorem 1.}

We shall look at part $(a)$ only, as part $(b)$ follows by part $(a)$ and the continuous mapping theorem. To that end, it suffices to examine the difference

$$
\frac{1}{\tilde{n}} \sum_{j=1}^{\mathbf{j}^{*}}\left\{\mathcal{T} \mathcal{P}_{n, \mathbf{B}}\left(\frac{\mathbf{b}^{*}}{\mathbf{B}} ; j\right)-\mathcal{T}_{n, \mathbf{B}}\left(\frac{\mathbf{b}^{*}}{\mathbf{B}} ; j\right)\right\}
$$

To that end, we first examine

$$
\frac{1}{\tilde{n} \mathbf{B}} \sum_{j=1}^{\mathbf{j}^{*}} \sum_{b=1}^{\mathbf{b}^{*}} \frac{I_{x, b}(j)}{f(j)}\left\{\frac{1}{\widehat{\sigma}_{\varepsilon}^{2}(b)}-1\right\}=\sum_{\ell=1}^{3} \Phi_{n, \ell}\left(\mathbf{j}^{*}, \mathbf{b}^{*}\right)+o_{p}\left(T^{-1 / 2}\right),
$$

where the right side is due to Taylor's expansion because Theorem 1 and $C 2$ imply that $\mathcal{E}\left\{\widehat{\sigma}_{\varepsilon}^{2}(b)-1\right\}^{4}=$ $O\left(\mathbf{B}^{-2}\right)=o\left(T^{-1 / 2}\right)$ and $\sup _{b=1, \ldots, \mathbf{B}}\left|\widehat{\sigma}_{\varepsilon}^{2}(b)-1\right|^{2}=o_{p}(\mathbf{B} / n)$ then yields that $o_{p}\left(T^{-1 / 2}\right)$ is uniformly in $\mathbf{j}^{*}$ and $\mathbf{b}^{*}$, and where

$$
\begin{aligned}
\Phi_{n, 1}\left(\mathbf{j}^{*}, \mathbf{b}^{*}\right) & =\frac{\mathbf{j}^{*}}{\tilde{n} \mathbf{B}} \sum_{b=1}^{\mathbf{b}^{*}} \sum_{k=1}^{3} \frac{(-1)^{k}}{k !}\left\{\widehat{\sigma}_{\varepsilon}^{2}(b)-1\right\}^{k} \\
\Phi_{n, 2}\left(\mathbf{j}^{*}, \mathbf{b}^{*}\right) & =\frac{1}{\tilde{n} \mathbf{B}} \sum_{j=1}^{\mathbf{j}^{*}} \sum_{b=1}^{\mathbf{b}^{*}} R_{n, b}(j) \sum_{k=1}^{3} \frac{(-1)^{k}}{k !}\left\{\widehat{\sigma}_{\varepsilon}^{2}(b)-1\right\}^{k} \\
\Phi_{n, 3}\left(\mathbf{j}^{*}, \mathbf{b}^{*}\right) & =\frac{1}{\tilde{n} \mathbf{B}} \sum_{j=1}^{\mathbf{j}^{*}} \sum_{b=1}^{\mathbf{b}^{*}} \stackrel{\circ}{I}_{\varepsilon, b}(j) \sum_{k=1}^{3} \frac{(-1)^{k}}{k !}\left\{\widehat{\sigma}_{\varepsilon}^{2}(b)-1\right\}^{k} .
\end{aligned}
$$


First we examine (5.19). The contribution due to the terms when $k=2,3$ is easily shown to satisfy (5.2) and hence Condition $B W$, and thus, we will only consider $k=1$, which is

$$
\frac{1}{\tilde{n} \mathbf{B}} \sum_{b=1}^{\mathbf{b}^{*}}\left\{\widetilde{\sigma}_{\varepsilon}^{2}(b)-\widehat{\sigma}_{\varepsilon}^{2}(b)\right\} \sum_{j=1}^{\mathbf{j}^{*}} \stackrel{\circ}{I}_{\varepsilon, b}(j)-\frac{1}{\tilde{n} \mathbf{B}} \sum_{b=1}^{\mathbf{b}^{*}}\left\{\widetilde{\sigma}_{\varepsilon}^{2}(b)-1\right\} \sum_{j=1}^{\mathbf{j}^{*}} \stackrel{\circ}{I}_{\varepsilon, b}(j)
$$

using the notation in (5.3).

Next, because $\mathcal{E}\left(\widetilde{\sigma}_{\varepsilon}^{2}(b)-1\right)^{4}=O\left(n^{-2}\right)$, the second term of (5.20) satisfies Condition $B W$ because

$$
\begin{aligned}
& \mathcal{E}\left|\frac{1}{\tilde{n} \mathbf{B}} \sum_{b=\mathbf{b}_{1}^{*}+1}^{\mathbf{b}_{2}^{*}}\left\{\tilde{\sigma}_{\varepsilon}^{2}(b)-1\right\} \sum_{j=\mathbf{j}_{1}^{*}+1}^{\mathbf{j}_{2}^{*}} \stackrel{\circ}{I}_{\varepsilon, b}(j)\right|^{2} \\
\leq & \frac{\mathbf{b}_{2}^{*}-\mathbf{b}_{1}^{*}}{\mathbf{B}^{2} n^{2}} \sum_{b=\mathbf{b}_{1}^{*}+1}^{\mathbf{b}_{2}^{*}} \mathcal{E}\left|\left\{\widetilde{\sigma}_{\varepsilon}^{2}(b)-1\right\} \sum_{j=\mathbf{j}_{1}^{*}+1}^{\mathbf{j}_{2}^{*}} \stackrel{\circ}{I}_{\varepsilon, b}(j)\right|^{2} \\
= & o_{p}\left(\frac{1}{T}\left(\frac{\mathbf{j}_{2}^{*}-\mathbf{j}_{1}^{*}}{\tilde{n}}\right)^{1+\delta}\left(\frac{\mathbf{b}_{2}^{*}-\mathbf{b}_{1}^{*}}{\mathbf{B}}\right)^{2}\right),
\end{aligned}
$$

by the Cauchy-Schwarz inequality and then Condition $C 2$.

Now, due to Lemma 7, the first term of $(5.20)$ is

$$
\frac{d_{n}}{\tilde{n} \mathbf{B}} \sum_{b=1}^{\mathbf{b}^{*}}\left(\frac{1}{n} \sum_{p=1}^{n} \breve{\psi}_{b, n}(p)+\frac{1}{\mathbf{B}}\right) \sum_{j=1}^{\mathbf{j}^{*}} \stackrel{\circ}{I}_{\varepsilon, b}(j)+\frac{\Psi_{n, 1}}{\tilde{n} \mathbf{B}} \sum_{b=1}^{\mathbf{b}^{*}} \Psi_{n, 2}(b)\left|\sum_{j=1}^{\mathbf{j}^{*}} \stackrel{\circ}{I}_{\varepsilon, b}(j)\right|,
$$

where using notation before Lemma 6 ,

$$
\breve{\psi}_{b, n}(p)=\mathcal{A}^{-1}(p) \psi_{1, n}(p) \stackrel{\circ}{I}_{\varepsilon, b}(p) .
$$

Because $\mathcal{E} \Psi_{n, 2}^{2}(b)=O\left(\mathbf{B}^{-3}+n^{-2}\right)$,

$$
\sup _{\mathbf{j}^{*}, \mathbf{b}^{*}}\left|\frac{1}{(\tilde{n} \mathbf{B})^{1 / 2}} \sum_{b=1}^{\mathbf{b}^{*}} \sum_{j=1}^{\mathbf{j}^{*}} \stackrel{\circ}{I}_{\varepsilon, b}(j)\right|=O_{p}(1) ; \quad \sup _{\mathbf{j}^{*}}\left|\frac{1}{\tilde{n}^{1 / 2}} \sum_{j=1}^{\mathbf{j}^{*}} \stackrel{\circ}{I}_{\varepsilon, b}(j)\right|=O_{p}(1)
$$

we can conclude then by $C 2$ that the first term of (5.20) satisfies condition $B W$ if the first term of (5.21) does. Therefore, we need to examine the behavior of

$$
\frac{1}{n} \sum_{p=1}^{\tilde{n}} \mathcal{A}^{-1}(p) \xi^{(1)}(p, j)+\frac{1}{n} \sum_{p=1}^{\tilde{n}} \mathcal{A}^{-1}(p) \xi^{(2)}(p, j),
$$

where

$$
\begin{aligned}
& \xi^{(1)}(p, j)=\sum_{\ell=1}^{n} \varsigma_{\ell p}\left(\frac{1}{\mathbf{B}} \sum_{v=\mathbf{b}_{1}^{*}+1}^{\mathbf{b}_{2}^{*}} \stackrel{\circ}{I}_{\varepsilon, v}(\ell)\right)\left\{\frac{1}{\tilde{n} \mathbf{B}} \sum_{b=\mathbf{b}_{1}^{*}+1}^{\mathbf{b}_{2}^{*}} \stackrel{\circ}{I}_{\varepsilon, b}(p) \sum_{j=\mathbf{j}_{1}^{*}+1}^{\mathbf{j}_{2}^{*}} \stackrel{\circ}{I}_{\varepsilon, b}(j)\right\} \\
& \xi^{(2)}(p, j)=\sum_{\ell=1}^{n} \varsigma_{\ell p}\left(\frac{1}{\mathbf{B}} \sum_{v \neq \mathbf{b}_{1}^{*}+1}^{\mathbf{b}_{2}^{*}} \stackrel{\circ}{I}_{\varepsilon, v}(\ell)\right)\left\{\frac{1}{\tilde{n} \mathbf{B}} \sum_{b=\mathbf{b}_{1}^{*}+1}^{\mathbf{b}_{2}^{*}} \stackrel{\circ}{I}_{\varepsilon, b}(p) \sum_{j=\mathbf{j}_{1}^{*}+1}^{\mathbf{j}_{2}^{*}} \stackrel{\circ}{I}_{\varepsilon, b}(j)\right\} .
\end{aligned}
$$


The second moment of $\xi^{(2)}(p, j)$ is

$$
\begin{aligned}
& \sum_{\ell_{1}, \ell_{2}=1}^{n} \varsigma_{\ell_{1} p} \varsigma_{\ell_{2} p}\left\{\frac{1}{\mathbf{B}^{2}} \sum_{v \neq \mathbf{b}_{1}^{*}+1}^{\mathbf{b}_{2}^{*}} \mathcal{E}\left(\stackrel{\circ}{I}_{\varepsilon, v}\left(\ell_{1}\right) \stackrel{\circ}{I}, v_{\varepsilon}\left(\ell_{2}\right)\right)\right\} \\
& \times \frac{1}{(\tilde{n} \mathbf{B})^{2}} \mathcal{E}\left\{\sum_{b=\mathbf{b}_{1}^{*}+1}^{\mathbf{b}_{2}^{*}} \stackrel{\circ}{I}_{\varepsilon, b}(p) \sum_{j=\mathbf{j}_{1}^{*}+1}^{\mathbf{j}_{2}^{*}} \stackrel{\circ}{I}, b_{\varepsilon}(j) \sum_{b=\mathbf{b}_{1}^{*}+1}^{\mathbf{b}_{2}^{*}} \stackrel{\circ}{I}_{\varepsilon, b}(p) \sum_{j=\mathbf{j}_{1}^{*}+1}^{\mathbf{j}_{2}^{*}} \stackrel{\circ}{I}_{\varepsilon, b}(j)\right\} \\
= & O\left(\frac{\log ^{2} n}{n \mathbf{B}^{2}}\left(\frac{\mathbf{b}_{2}^{*}-\mathbf{b}_{1}^{*}}{\mathbf{B}}\right)^{2}\left(\frac{\mathbf{j}_{2}^{*}-\mathbf{j}_{1}^{*}}{\tilde{n}}\right)\right)
\end{aligned}
$$

by Condition $C 1$ and using (5.55) in Lemma 7 . However, $\xi^{(1)}(p, j)$ is

$$
\begin{aligned}
& \frac{1}{\tilde{n} \mathbf{B}^{2}} \sum_{v=\mathbf{b}_{1}^{*}+1}^{\mathbf{b}_{2}^{*}}\left(\sum_{\ell=1}^{n} \varsigma_{\ell p} \stackrel{\circ}{\varepsilon}, v_{\varepsilon}(\ell) \stackrel{\circ}{I}_{\varepsilon, v}(p)\right)\left\{\sum_{j=\mathbf{j}_{1}^{*}+1}^{\mathbf{j}_{2}^{*}} \stackrel{\circ}{I}, v^{\prime}(j)\right\} \\
& +\frac{1}{\tilde{n} \mathbf{B}^{2}} \sum_{v \neq b=\mathbf{b}_{1}^{*}+1}^{\mathbf{b}_{2}^{*}}\left(\sum_{\ell=1}^{n} \varsigma_{\ell p} \stackrel{\circ}{I}_{\varepsilon, v}(\ell) \stackrel{\circ}{I}_{\varepsilon, b}(p)\right)\left\{\sum_{j=\mathbf{j}_{1}^{*}+1}^{\mathbf{j}_{2}^{*}} \stackrel{\circ}{I}_{\varepsilon, b}(j)\right\} .
\end{aligned}
$$

The first term of the last displayed expression satisfies Condition $B W$ by a routine use of the CauchySchwarz inequality, whereas Condition $C 1$ implies that the second moment of the second term is

$$
\begin{aligned}
& \frac{1}{\tilde{n}^{2} \mathbf{B}^{4}} \sum_{\ell_{1}, \ell_{2}=1}^{n} \varsigma_{\ell_{1} p} \varsigma_{\ell_{2} p}\left\{\sum_{v=\mathbf{b}_{1}^{*}+1}^{\mathbf{b}_{2}^{*}} \mathcal{E}\left(\stackrel{\circ}{I}_{\varepsilon, v}\left(\ell_{1}\right) \stackrel{\circ}{\varepsilon}, v_{\varepsilon}\left(\ell_{2}\right)\right)\right. \\
& \left.\times\left(\sum_{\substack{b_{1}, b_{2}=\mathbf{b}_{1}^{*}+1 \\
b_{1}, b_{2} \neq v}}^{\mathbf{b}_{2}^{*}} \mathcal{E}\left(\stackrel{\circ}{I}_{\varepsilon, b_{1}}(p) \stackrel{\circ}{I}_{\varepsilon, b_{2}}(p)\left\{\sum_{j=\mathbf{j}_{1}^{*}+1}^{\mathbf{j}_{2}^{*}} \stackrel{\circ}{I}_{\varepsilon, b_{1}}(j)\right\}\left\{\sum_{j=\mathbf{j}_{1}^{*}+1}^{\mathbf{j}_{2}^{*}}{\stackrel{\circ}{I}, b_{2}}^{*}(j)\right\}\right)\right)\right\} \\
& =\frac{\mathbf{b}_{2}^{*}-\mathbf{b}_{1}^{*}}{\tilde{n}^{2} \mathbf{B}^{4}}\left\{\sum_{\ell_{1}, \ell_{2}=1}^{n} \varsigma_{\ell_{1} p} \varsigma_{\ell_{2} p}\left(\mathcal{I}\left(\ell_{1}=\ell_{2}\right)+\frac{\kappa_{4}}{n}\right) \times\right. \\
& \left\{\left(\sum_{b=\mathbf{b}_{1}^{*}+1}^{\mathbf{b}_{2}^{*}} \mathcal{E}\left(\stackrel{\circ}{I}_{\varepsilon, b}(p) \sum_{j=\mathbf{j}_{1}^{*}+1}^{\mathbf{j}_{2}^{*}} \stackrel{\circ}{I}_{\varepsilon, b}(j)\right)\right)^{2}+\sum_{b=\mathbf{b}_{1}^{*}+1}^{\mathbf{b}_{2}^{*}} \mathcal{E}\left(\stackrel{\circ}{I}_{\varepsilon, b}^{2}(p)\right) \mathcal{E}\left(\sum_{j=\mathbf{j}_{1}^{*}+1}^{\mathbf{j}_{2}^{*}} \stackrel{\circ}{I}_{\varepsilon, b}(j)\right)^{2}\right. \\
& \left.+\sum_{b=\mathbf{b}_{1}^{*}+1}^{\mathbf{b}_{2}^{*}} \sum_{j, k=\mathbf{j}_{1}^{*}+1}^{\mathbf{j}_{2}^{*}} \operatorname{cum}\left(\stackrel{\circ}{I}_{\varepsilon, b}(p) ; \stackrel{\circ}{I}_{\varepsilon, b}(p) ; \stackrel{\circ}{I}_{\varepsilon, b}(j) ; \stackrel{\circ}{I}_{\varepsilon, b}(k)\right)\right\} .
\end{aligned}
$$

From here and a standard used of (5.55) of Lemma 7, it follows that it satisfies Condition $B W$. Thus, this demonstrates that (5.24) and hence that the first term of (5.20) satisfies (5.2), i.e., Condition $B W$.

Next, (5.18). As with (5.19), the contribution due to the terms when $k=2,3$ satisfies (5.2) with $\alpha=1$ there and, hence, Condition $B W$. Thus, we examine

$$
\frac{1}{\tilde{n} \mathbf{B}} \sum_{j=1}^{\mathbf{j}^{*}} \sum_{b=1}^{\mathbf{b}^{*}} R_{n, b}(j)\left(\widehat{\sigma}_{\varepsilon}^{2}(b)-1\right) .
$$

Using (5.29) and the definition in (5.31), we have that it suffices to show (5.2) for

$$
\frac{1}{\tilde{n} \mathbf{B}} \sum_{j=1}^{\mathbf{j}^{*}} \sum_{b=1}^{\mathbf{b}^{*}} \mathrm{Z}_{n, b}^{(1)}(-j)\left(\widehat{\sigma}_{\varepsilon}^{2}(b)-1\right) \text {. }
$$


because $\mathcal{E}\left|\mathrm{Y}_{n, b}(j ; 0)\right|^{4}+E\left|\mathrm{Z}_{n, b}^{(2)}(-j)\right|^{2}=O\left(n^{-2}\right)$ and then Theorem 1 and Condition $C 2$. Now, because $\mathcal{E} \mathrm{Z}_{n, b}^{(1)}(-j)=O\left(n^{-1}\right)$, it implies that it suffices to show that

$$
\begin{aligned}
& \frac{1}{\tilde{n} \mathbf{B}} \sum_{j=1}^{\mathbf{j}^{*}} \sum_{b=1}^{\mathbf{b}^{*}}\left(\mathrm{Z}_{n, b}^{(1)}(-j)-\mathcal{E} \mathrm{Z}_{n, b}^{(1)}(-j)\right)\left(\widehat{\sigma}_{\varepsilon}^{2}(b)-\widetilde{\sigma}_{\varepsilon}^{2}(b)\right) \\
& +\frac{1}{\tilde{n} \mathbf{B}} \sum_{j=1}^{\mathbf{j}^{*}} \sum_{b=1}^{\mathbf{b}^{*}}\left(\mathrm{Z}_{n, b}^{(1)}(-j)-\mathcal{E} \mathrm{Z}_{n, b}^{(1)}(-j)\right)\left(\widetilde{\sigma}_{\varepsilon}^{2}(b)-1\right)
\end{aligned}
$$

satisfies (5.2) and hence Condition $B W$. Clearly, the second term of the last displayed expression satisfies (5.2) using Lemma 1 part $(\mathbf{a})$ and that $\mathcal{E}\left(\widetilde{\sigma}_{\varepsilon}^{2}(b)-1\right)^{2}=O\left(n^{-1}\right)$, whereas the first term proceeding as with $(5.21)$ is

$$
\frac{1}{\tilde{n} \mathbf{B}} \sum_{j=1}^{\mathbf{j}^{*}} \sum_{b=1}^{\mathbf{b}^{*}}\left(\mathrm{Z}_{n, b}^{(1)}(-j)-\mathcal{E} \mathrm{Z}_{n, b}^{(1)}(-j)\right) \frac{1}{n} \sum_{p=1}^{n} \breve{\psi}_{b, n}(p)+o_{p}\left(T^{-1 / 2}\right)
$$

uniformly in $\mathbf{j}^{*}$ and $\mathbf{b}^{*}$. Then, proceed step by step as with $(5.24)$ but with $\stackrel{\circ}{\varepsilon}, b(j)$ replaced with $\mathrm{Z}_{n, b}^{(1)}(-j)-\mathcal{E} \mathrm{Z}_{n, b}^{(1)}(-j)$.

Thus, it remains to examine the behavior of (5.17), which is

$$
\frac{\mathbf{j}^{*}}{\tilde{n} \mathbf{B}} \sum_{b=1}^{\mathbf{b}^{*}}\left\{\widehat{\sigma}_{\varepsilon}^{2}(b)-1\right\}+\frac{\mathbf{j}^{*}}{\tilde{n} \mathbf{B}} \sum_{b=1}^{\mathbf{b}^{*}} \sum_{k=2}^{3} \frac{(-1)^{k}}{k !}\left\{\hat{\sigma}_{\varepsilon}^{2}(b)-1\right\}^{k}
$$

We first examine the second term of (5.25). The contribution due to $\widetilde{\sigma}_{\varepsilon}^{2}(b)-1$ is $o_{p}\left(T^{-1 / 2}\right)$, uniformly in $\mathbf{j}^{*}$ and $\mathbf{b}^{*}$ because

$$
\begin{aligned}
\mathcal{E} \sup _{\mathbf{j}^{*} ; \mathbf{b}^{*}}\left|\frac{\mathbf{j}^{*}}{\tilde{n} \mathbf{B}} \sum_{b=1}^{\mathbf{b}^{*}} \sum_{k=2}^{3}\left\{\widetilde{\sigma}_{\varepsilon}^{2}(b)-1\right\}^{k}\right| & \leq \frac{1}{\mathbf{B}} \sum_{b=1}^{\mathbf{B}} \sum_{k=2}^{3} \mathcal{E}\left|\widetilde{\sigma}_{\varepsilon}^{2}(b)-1\right|^{k} \\
& =O\left(n^{-1}\right)=o\left(T^{-1 / 2}\right),
\end{aligned}
$$

and thus, it is the contribution due to $\frac{d_{2, n}}{\mathrm{~B}}+\Psi_{n, 1} \Psi_{n, 2}(b)$ because by Lemma $7, \mathcal{E}\left|\frac{d_{2, n}}{\mathrm{~B}}+\Psi_{n, 2}(b)\right|^{k}=$ $o_{p}\left(T^{-1 / 2}\right)$ and $\Psi_{n, 1}=O_{p}(1)$. Next, the contribution due to $\frac{1}{n} \sum_{p=1}^{n} \breve{\psi}_{b, n}(p)$, that is,

$$
\frac{\mathbf{j}^{*}}{\tilde{n} \mathbf{B}} \sum_{b=1}^{\mathbf{b}^{*}} \sum_{k=2}^{3} \frac{(-1)^{k}}{k !}\left(\frac{1}{n} \sum_{p=1}^{n} \mathcal{A}^{-1}(p) \psi_{1, n}(p) \stackrel{\circ}{I}_{\varepsilon, b}(p)\right)^{k} .
$$


Now,

$$
\begin{aligned}
& \mathcal{E} \sup _{\mathbf{j}^{*} ; \mathbf{b}^{*}}\left|\frac{\mathbf{j}^{*}}{\tilde{n} \mathbf{B}} \sum_{b=1}^{\mathbf{b}^{*}} \sum_{k=2}^{3}\left\{\frac{1}{n} \sum_{p=1}^{n} \mathcal{A}^{-1}(p) \psi_{1, n}(p) \stackrel{\circ}{I}_{\varepsilon, b}(p)\right\}^{k}\right| \\
= & \frac{C}{\mathbf{B}} \sum_{b=1}^{\mathbf{B}}\left\{\mathcal{E}\left(\frac{1}{n} \sum_{p=1}^{n} \mathcal{A}^{-1}(p) \psi_{1, n}(p) \stackrel{\circ}{I}_{\varepsilon, b}(p)\right)^{2}+\frac{1}{n} \sum_{p=1}^{n} \mathcal{E}\left(\left|\psi_{1, n}(p)\right|^{3}\left|\stackrel{\circ}{I}_{\varepsilon, b}(p)\right|^{3}\right)\right\} \\
\leq & \frac{C}{\mathbf{B}} \sum_{b=1}^{\mathbf{B}}\left\{\mathcal{E}\left(\frac{1}{n \mathbf{B}} \sum_{p=1}^{n} \mathcal{A}^{-1}(p) \stackrel{\circ}{I}_{\varepsilon, b}(p) \sum_{\ell=1}^{n} \varsigma_{\ell p} \sum_{v \neq b} \stackrel{\circ}{I}_{\varepsilon, v}(\ell)\right)^{2}\right\} \\
& +\frac{C}{\mathbf{B}} \sum_{b=1}^{\mathbf{B}}\left\{\mathcal{E}\left(\frac{1}{n \mathbf{B}} \sum_{p=1}^{n} \mathcal{A}^{-1}(p) \stackrel{\circ}{I}_{\varepsilon, b}(p) \sum_{\ell=1}^{n} \varsigma_{\ell p} \stackrel{\circ}{I}_{\varepsilon, b}(\ell)\right)^{2}\right\}+O\left(\frac{1}{\mathbf{B}^{3 / 2}}\right) \\
= & o_{p}\left(T^{-1 / 2}\right)
\end{aligned}
$$

because $\mathbf{B}^{-3 / 2}=o\left(T^{-1 / 2}\right)$ by $C 2$ and using (5.55). Thus, we have that the second term of (5.25) satisfies Condition $B W$.

Next, the first term of (5.25), which is using (5.22)

$$
\frac{\mathbf{j}^{*}}{\tilde{n} \mathbf{B}} \sum_{b=1}^{\mathbf{b}^{*}}\left\{\widetilde{\sigma}_{\varepsilon}^{2}(b)-1\right\}+\frac{\mathbf{j}^{*}}{\tilde{n} \mathbf{B}} \sum_{b=1}^{\mathbf{b}^{*}} \frac{1}{n} \sum_{j=1}^{n} \breve{\psi}_{b, n}(j)+\frac{\mathbf{j}^{*} \mathbf{b}^{*}}{\tilde{n} \mathbf{B}}\left(\frac{d_{2, n}}{\mathbf{B}}\right)+o_{p}\left(\frac{1}{T^{1 / 2}}\right) .
$$

by Lemma 7 . The second term is

$$
\begin{aligned}
& \frac{\mathbf{j}^{*}}{\tilde{n} \mathbf{B}} \sum_{b=1}^{\mathbf{b}^{*}} \frac{1}{n} \sum_{j=1}^{n} \sum_{p=1}^{n} \varsigma_{j p}\left(\frac{1}{\mathbf{B}} \sum_{v \neq b} \stackrel{\circ}{I}_{\varepsilon, v}(p)\right) \stackrel{\circ}{I}_{\varepsilon, b}(j) \\
& +\frac{\mathbf{j}^{*}}{\tilde{n} \mathbf{B}} \sum_{p=1}^{n} \varsigma_{j p} \frac{1}{n \mathbf{B}} \sum_{j=1}^{n} \sum_{b=1}^{\mathbf{b}^{*}} \stackrel{\circ}{I}_{\varepsilon, b}(j) \stackrel{\circ}{I}_{\varepsilon, b}(p) .
\end{aligned}
$$

Again, because

$$
\left(\frac{1}{n \mathbf{B}} \sum_{j=1}^{n} \sum_{b=1}^{\mathbf{b}^{*}} \stackrel{\circ}{I}_{\varepsilon, b}(j) \stackrel{\circ}{I}_{\varepsilon, b}(p)\right)^{2}=O\left(T^{-1 / 2}\right)
$$

it suffices to examine

$$
\frac{1}{\mathbf{B}} \sum_{b=1}^{\mathbf{b}^{*}} \frac{1}{n} \sum_{j=1}^{n} \sum_{p=1}^{n} \varsigma_{j p}\left(\frac{1}{\mathbf{B}} \sum_{v \neq b} \stackrel{\circ}{I}_{\varepsilon, v}(p)\right) \stackrel{\circ}{I}_{\varepsilon, b}(j)
$$

from our comments made after (5.19). The second moments are

$$
\frac{1}{\mathbf{B}^{4}} \frac{1}{n^{2}} \sum_{j_{1}, j_{2}=1}^{n} \sum_{p_{1}, p_{2}=1}^{n} \varsigma_{j_{1} p_{1}} \varsigma_{j_{2} p_{2}} \sum_{b=1}^{\mathbf{b}^{*}}\left\{\mathcal{E}\left(\stackrel{\circ}{I}_{\varepsilon, b}\left(j_{1}\right) \stackrel{\circ}{I}_{\varepsilon, b}\left(j_{2}\right)\right) \sum_{v \neq b} \mathcal{E}\left(\stackrel{\circ}{I}_{\varepsilon, v}\left(p_{1}\right) \stackrel{\circ}{I}_{\varepsilon, v}\left(p_{2}\right)\right)\right\} .
$$

Now, use (5.55) to conclude that it is $o\left(T^{-1 / 2}\right)$.

We have obtained that, uniformly in $\mathbf{b}^{*}$ and $\mathbf{j}^{*},(5.16)$ is

$$
\frac{\mathbf{j}^{*}}{\tilde{n}} \frac{1}{\mathbf{B}} \sum_{b=1}^{\mathbf{b}^{*}}\left(\widetilde{\sigma}_{\varepsilon}^{2}(b)-1\right)+\frac{\mathbf{j}^{*} \mathbf{b}^{*}}{\tilde{n} \mathbf{B}}\left(\frac{d_{2, n}}{\mathbf{B}}\right)+o_{p}\left(\frac{1}{T^{1 / 2}}\right)
$$


and hence, (5.15) becomes

$$
\frac{\mathbf{j}^{*}}{\tilde{n}}\left\{\frac{1}{\mathbf{B}} \sum_{b=1}^{\mathbf{b}^{*}}\left(\widetilde{\sigma}_{\varepsilon}^{2}(b)-1\right)-\frac{\mathbf{b}^{*}}{\mathbf{B}} \frac{1}{\mathbf{B}} \sum_{b=1}^{\mathbf{B}}\left(\widetilde{\sigma}_{\varepsilon}^{2}(b)-1\right)\right\}+o_{p}\left(\frac{1}{T^{1 / 2}}\right)
$$

proceeding as in the proof of Proposition 1 and because the second term of (5.26) is independent of $b$. From here, we then conclude that

$$
\begin{aligned}
{[T / 2] \mathcal{T} \mathcal{P}_{n, \mathbf{B}}\left(\frac{\mathbf{j}^{*}}{\tilde{n}}, \frac{\mathbf{b}^{*}}{\mathbf{B}}\right)=\frac{1}{(\tilde{n} \mathbf{B})^{1 / 2}} \sum_{j=1}^{\mathbf{j}^{*}} \sum_{b=1}^{\mathbf{b}^{*}}\left\{\stackrel{\circ}{I}_{\varepsilon, b}(j)-\frac{1}{n} \sum_{t=1}^{n}\left(\varepsilon_{t+(b-1) n}^{2}-1\right)\right.} \\
\left.-\left(\overline{\check{I}}_{\varepsilon}(j)-\frac{1}{T} \sum_{t=1}^{T}\left(\varepsilon_{t}^{2}-1\right)\right)\right\}+o_{p}(1)
\end{aligned}
$$

using the proof of Proposition 1

However, standard algebra indicates that

$$
\begin{aligned}
& \mathcal{E}\left(\stackrel{\circ}{I}_{\varepsilon, b}(j)-\frac{1}{n} \sum_{t=1}^{n}\left(\varepsilon_{t+(b-1) n}^{2}-1\right) ; \stackrel{\circ}{I}_{\varepsilon, b}(-k)-\frac{1}{n} \sum_{t=1}^{n}\left(\varepsilon_{t+(b-1) n}^{2}-1\right)\right) \\
= & \frac{1}{n^{2}} \sum_{t_{1} \neq s_{1} ; t_{2} \neq s_{2}} \mathcal{E}\left(\varepsilon_{t_{1}} \varepsilon_{s_{1}} \varepsilon_{t_{2}} \varepsilon_{s_{2}}\right) e^{i\left(t_{1}-s_{1}\right) \lambda_{j}-i\left(t_{2}-s_{2}\right) \lambda_{k}} \\
= & \mathcal{I}(j=k)-\frac{2}{n} .
\end{aligned}
$$

The proof now follows by routine arguments, and thus, they are omitted.

\subsection{Proof of Proposition 3.}

We examine part $(a)$, and part (b) follows identically using Theorem 1 and $\sigma_{\varepsilon}^{2}(b)=\sigma_{\varepsilon}^{2}$. The proof is similar to that of Proposition 2 but we employ Lemmas 4 and 5 instead of Lemmas 2 and 3 when needed. Abbreviating $f\left(\frac{b n}{T} ; j\right) / \bar{f}_{\mathbf{B}}(j)$ as $\ddot{f}\left(\frac{b n}{T} ; j\right)$, where $f\left(\frac{b n}{T} ; j\right)=\left(1+g(b n / T ; j) /[T / 2]^{1 / 2}\right)$ and $\bar{f}_{\mathbf{B}}(j)=\mathbf{B}^{-1} \sum_{b=1}^{\mathbf{B}} f\left(\frac{b n}{T} ; j\right)$, we easily seethat $\mathcal{T}_{n, \mathbf{B}}\left(\frac{\mathbf{b}^{*}}{\mathbf{B}} ; \mathbf{j}_{\tilde{n}}^{*}\right)$ is

$$
\frac{1}{\tilde{n}} \sum_{j=1}^{\mathbf{j}^{*}}\left\{\frac{1}{\mathbf{B}} \sum_{b=1}^{\mathbf{b}^{*}}\left(\frac{\ddot{f}\left(\frac{b n}{T} ; j\right) \stackrel{\circ}{I}_{\varepsilon, b}(j)+\ddot{f}\left(\frac{b n}{T} ; j\right)}{\mathbf{B}^{-1} \sum_{b=1}^{\mathbf{B}} \ddot{f}\left(\frac{b n}{T} ; j\right) \stackrel{\circ}{I}_{\varepsilon, b}(j)+1}-1\right)\right\} 1+o_{p}\left(T^{-1 / 2}\right) .
$$

Now, using Taylor's expansion of $x^{-1}$ around 1 and the arguments in the proof of Proposition 1 , we have that

$$
\frac{1}{\mathbf{B}^{-1} \sum_{b=1}^{\mathbf{B}} \ddot{f}\left(\frac{b n}{T} ; j\right) \stackrel{\circ}{I}, b_{\varepsilon}(j)+1} \stackrel{\text { asym }}{\simeq} 1-v_{n}(j)+v_{n}^{2}(j),
$$

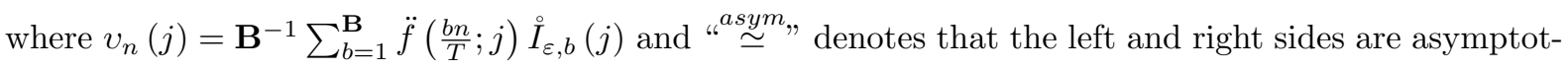
ically equivalent.

Therefore, the asymptotic behavior of $[T / 2]^{1 / 2} \mathcal{T}_{n, \mathbf{B}}\left(\frac{\mathbf{b}^{*}}{\mathbf{B}} ; \frac{\mathbf{j}^{*}}{\tilde{n}}\right)$ is governed by

$$
\begin{aligned}
& {[T / 2]^{1 / 2} \frac{1}{\tilde{n}} \sum_{j=1}^{\mathbf{j}^{*}}\left\{\frac{1}{\mathbf{B}} \sum_{b=1}^{\mathbf{b}^{*}}\left\{\left(\ddot{f}\left(\frac{b n}{T} ; j\right) \stackrel{\circ}{I}_{\varepsilon, b}(j)-v_{n}(j)\right)+\left(\ddot{f}\left(\frac{b n}{T} ; j\right)-1\right)\right\}\right.} \\
& \left.\times\left(1-v_{n}(j)+v_{n}^{2}(j)\right)\right\} .
\end{aligned}
$$


Now, except the terms of smaller orders of magnitude, the expectation of the last displayed expression is

$$
\begin{aligned}
& {[T / 2]^{1 / 2} \frac{1}{\tilde{n}} \sum_{j=1}^{\mathbf{j}^{*}} \frac{1}{\mathbf{B}} \sum_{b=1}^{\mathbf{b}^{*}}\left(\ddot{f}\left(\frac{b n}{T} ; j\right)-1\right) } \\
= & \frac{1}{\tilde{n}} \sum_{j=1}^{\mathbf{j}^{*}} \frac{1}{\mathbf{B}} \sum_{b=1}^{\mathbf{b}^{*}}\left\{g\left(\frac{b n}{T} ; j\right)-\frac{1}{\mathbf{B}} \sum_{b=1}^{\mathbf{B}} g\left(\frac{b n}{T} ; j\right)\right\}_{T, n \rightarrow_{\infty}}^{\rightarrow} d\left(\omega^{*} ; v^{*}\right)
\end{aligned}
$$

using the definition of $\ddot{f}\left(\frac{b n}{T} ; j\right)$ under $H_{l}$. Now, the proof of the proposition proceeds as that of Proposition 2 and is thus omitted.

Let us introduce some notation. In what follows, we denote

$$
\begin{aligned}
\stackrel{\circ}{I}_{\varepsilon^{*}, b}(j) & =I_{\varepsilon^{*}, b}(j)-1 ; \bar{I}_{\varepsilon^{*}}(j)=\frac{1}{\mathbf{B}} \sum_{b=1}^{\mathbf{B}} I_{\varepsilon^{*}, b}(j) \\
\overline{\stackrel{I}{I}}_{\varepsilon^{*}}(j) & =\frac{1}{\mathbf{B}} \sum_{b=1}^{\mathbf{B}} \stackrel{\circ}{I}_{\varepsilon^{*}, b}(j) .
\end{aligned}
$$

Notice that $\mathcal{E}^{*}\left(\stackrel{\circ}{I}_{\mathcal{E}^{*}, b}(j)\right)=0$. Moreover $\left\{H_{n}\right\}_{n \geq 1}$ is a sequence of strictly positive $O_{p}(1)$ random variables.

\subsection{Proof of Theorem 2 .}

We will address only part $(a)$, as part $(b)$ follows similarly. We need to show that

$$
[T / 2]^{1 / 2} \mathcal{T}_{n, \mathbf{B}}^{*}\left(\frac{\mathbf{b}^{*}}{\mathbf{B}} ; \frac{\mathbf{j}^{*}}{\tilde{n}}\right) \stackrel{\text { weakly }}{\Rightarrow} \mathcal{B S}\left([0,1]^{2}\right) \quad \text { (in probability) }
$$

Now, using Taylor's expansion of $\bar{I}_{\varepsilon^{*}}^{-1}(j)$ around 1 , we obtain the following decomposition of $\mathcal{T}_{n, \mathbf{B}}^{*}\left(\frac{\mathbf{j}^{*}}{\tilde{n}}, \frac{\mathbf{b}^{*}}{\mathbf{B}}\right)$

$$
\begin{aligned}
& \frac{1}{\tilde{n}} \sum_{j=1}^{\mathbf{j}^{*}} \frac{1}{\mathbf{B}} \sum_{b=1}^{\mathbf{b}^{*}}\left(\stackrel{\circ}{I}_{\varepsilon^{*}, b}(j)-\overline{\bar{I}}_{\varepsilon^{*}}(j)\right) \overline{\stackrel{I}{I}}_{\varepsilon^{*}}(j) O_{p^{*}}(1) \\
& +\frac{1}{\tilde{n}} \sum_{j=1}^{\mathbf{j}^{*}}\left(\frac{1}{\mathbf{B}} \sum_{b=1}^{\mathbf{b}^{*}}\left\{\stackrel{\circ}{I}_{\varepsilon^{*}, b}(j)-\overline{\bar{I}}_{\varepsilon^{*}}(j)\right\}\right) \sum_{k=0}^{2} \frac{(-1)^{k} \overline{\bar{I}}_{\varepsilon^{*}}^{k}(j) .}{k !}
\end{aligned}
$$

Notice that Lemma 9 yields that $\left(\sup _{j} \overline{\bar{I}}_{\varepsilon^{*}}(j)\right)^{4} \leq \sum_{j=1}^{\tilde{n}} \overline{\stackrel{\oplus}{I}}_{\varepsilon^{*}}^{4}(j)=o_{p^{*}}(1)$ by $C 1$, such that $\bar{I}_{\varepsilon^{*}}^{-1}(j)<H_{n}$. Thus, proceeding as in the proof of Proposition 1but using now Lemma 9, we easily conclude that $\mathcal{T}_{n, \mathbf{B}}^{*}\left(\frac{\mathbf{j}^{*}}{\tilde{n}}, \frac{\mathbf{b}^{*}}{\mathbf{B}}\right)$ is governed by

$$
\frac{1}{\tilde{n} \mathbf{B}} \sum_{j=1}^{\mathbf{j}^{*}} \sum_{b=1}^{\mathbf{b}^{*}}\left\{\stackrel{\circ}{I}_{\varepsilon^{*}, b}(j)-\overline{\stackrel{\circ}{I}}_{\varepsilon^{*}}(j)\right\} \sum_{k=0}^{2} \frac{(-1)^{k}}{k !} \overline{\bar{I}}_{\varepsilon^{*}}^{k}(j) .
$$

Next, we examine the contribution due to $k=1,2$ in (5.27) and in particular

$$
\frac{1}{\tilde{n}} \sum_{j=1}^{\mathbf{j}^{*}}\left(\frac{1}{\mathbf{B}} \sum_{b=1}^{\mathbf{b}^{*}} \stackrel{\circ}{I}_{\varepsilon^{*}, b}(j)\right) \sum_{k=1}^{2} \stackrel{\bar{\circ}}{I}_{\varepsilon^{*}}(j) \text {. }
$$


To that end, we write

$$
\begin{aligned}
\frac{1}{\mathbf{B}} \sum_{b=1}^{\mathbf{B}}{\stackrel{\circ}{\varepsilon^{*}, b}}(j) & =\frac{1}{\mathbf{B}}\left\{\sum_{b=1}^{\mathbf{b}_{1}^{*}}+\sum_{b=\mathbf{b}_{2}^{*}+1}^{\mathbf{B}}\right\}{\stackrel{\circ}{\varepsilon^{*}, b}}(j)+\frac{1}{\mathbf{B}} \sum_{b=\mathbf{b}_{1}^{*}+1}^{\mathbf{b}_{2}^{*}}{\stackrel{\circ}{\varepsilon^{*}, b}}(j) \\
& =: \overline{\dot{I}}_{\varepsilon^{*}}(j)+\overline{\bar{I}}_{\varepsilon^{*}}(j) .
\end{aligned}
$$

Now, because ${\stackrel{\circ}{\varepsilon^{*}, b_{1}}}(j)$ and ${\stackrel{\circ}{\varepsilon^{*}, b_{2}}}(k)$ are independent for all $j, k$ when $b_{1} \neq b_{2}$, we have that

$$
\begin{aligned}
& \mathcal{E}^{*}\left(\stackrel{\circ}{I}_{\varepsilon^{*}, b_{1}}(j){\stackrel{\circ}{\varepsilon^{*}, b_{2}}}(k)\right)=0 \text { if } b_{1} \neq b_{2} \\
& \mathcal{E}^{*}\left({\stackrel{\circ}{I} \varepsilon^{*}, b}(j){\stackrel{\circ}{I_{\varepsilon^{*}, b}}}_{(k)}(k)=\widehat{\sigma}_{\varepsilon}^{2}(b) \mathcal{I}(j=k)+\frac{1}{n} \operatorname{Cum}^{*}\left(\varepsilon_{t}^{*} ; \varepsilon_{t}^{*} ; \varepsilon_{t}^{*} ; \varepsilon_{t}^{*}\right),\right.
\end{aligned}
$$

by standard arguments. Thus, the latter two displayed expressions imply that $\overline{\dot{I}}_{\varepsilon^{*}}(j)$ and $\overline{\bar{I}}_{\varepsilon^{*}}(k)$ are independent and hence

$$
\begin{aligned}
& \mathcal{E}^{*}\left(\frac{1}{\tilde{n}} \sum_{j=\mathbf{j}_{1}^{*}+1}^{\mathbf{j}_{2}^{*}} \overline{\ddot{I}}_{\mathcal{E}^{*}}(j) \sum_{k=1}^{2} \overline{\bar{I}}_{\mathcal{E}^{*}}^{k}(j)\right)^{2} \\
= & \frac{1}{\tilde{n}^{2}} \sum_{j, k=\mathbf{j}_{1}^{*}+1}^{\mathbf{j}_{2}^{*}}\left(\frac{1}{\overline{\mathbf{B}}^{2}} \sum_{b=\mathbf{b}_{1}^{*}+1}^{\mathbf{b}_{2}^{*}} \mathcal{E}^{*}\left(\stackrel{\circ}{I}_{\mathcal{E}^{*}, b}(j){\stackrel{\circ}{\mathcal{E}^{*}, b}}_{(}(k)\right)\right) \mathcal{E}^{*}\left(\sum_{k=1}^{2} \bar{I}_{\mathcal{E}^{*}}^{k}(j)\right)\left(\sum_{k=1}^{2} \bar{I}_{\mathcal{E}^{*}}^{k}(k)\right) \\
= & o\left(T^{-1}\left(\frac{\mathbf{b}_{2}^{*}-\mathbf{b}_{1}^{*}}{\mathbf{B}}\right)^{2}\left(\frac{\mathbf{j}_{2}^{*}-\mathbf{j}_{1}^{*}}{\tilde{n}}\right)^{2}\right) H_{n} .
\end{aligned}
$$

Moreover we have that $\mathcal{E}^{*}\left(\sum_{j=\mathbf{j}_{1}^{*}+1}^{\mathbf{j}_{2}^{*}} \overline{\ddot{I}}_{\varepsilon^{*}}^{2}(j) \overline{\dot{I}}_{\varepsilon^{*}}(j)\right)^{2}=0$.

Next, to finish the contribution due to $k=1,2$ in $(5.27)$, we need to examine

$$
\begin{aligned}
& \mathcal{E}^{*}\left(\frac{1}{\tilde{n}} \sum_{j=\mathbf{j}_{1}^{*}+1}^{\mathbf{j}_{2}^{*}} \overline{\bar{I}}_{\varepsilon^{*}}(j) \sum_{k=1}^{2} \bar{I}_{\mathcal{E}^{*}}^{k}(j)\right)^{2} \\
= & \mathcal{E}^{*}\left(\sum_{k=1}^{2} \frac{1}{\tilde{n}} \sum_{j=\mathbf{j}_{1}^{*}+1}^{\mathbf{j}_{2}^{*}} \bar{I}_{\varepsilon^{*}}^{k+1}(j)\right)^{2} \\
= & \mathcal{E}^{*}\left(\frac{1}{\tilde{n}} \sum_{j=\mathbf{j}_{1}^{*}+1}^{\mathbf{j}_{2}^{*}} \bar{I}_{\varepsilon^{*}}^{2}(j)\right)^{2}+o\left(T^{-1}\left(\frac{\mathbf{b}_{2}^{*}-\mathbf{b}_{1}^{*}}{\mathbf{B}}\right)^{2}\left(\frac{\mathbf{j}_{2}^{*}-\mathbf{j}_{1}^{*}}{\tilde{n}}\right)^{2}\right) H_{n}
\end{aligned}
$$

because by Condition $C 2, \mathbf{B}^{-3}=o\left(T^{-1}\right)$ and Lemma 9 . However, by Lemma 9 and standard arguments, the right side of the last displayed expression is

$$
\frac{1}{\mathbf{B}^{2}}\left(\frac{\mathbf{b}_{2}^{*}-\mathbf{b}_{1}^{*}}{\mathbf{B}}\right)^{2}\left(\frac{\mathbf{j}_{2}^{*}-\mathbf{j}_{1}^{*}}{\tilde{n}}\right)^{2} \mathcal{E}^{*} I_{\mathcal{E}^{*}}^{2}(j)+o\left(T^{-1}\left(\frac{\mathbf{b}_{2}^{*}-\mathbf{b}_{1}^{*}}{\mathbf{B}}\right)^{2}\left(\frac{\mathbf{j}_{2}^{*}-\mathbf{j}_{1}^{*}}{\tilde{n}}\right)^{2}\right) H_{n} .
$$

Now, proceeding similarly with

$$
\frac{1}{\tilde{n}} \sum_{j=1}^{\mathbf{j}^{*}}\left(\frac{\mathbf{b}^{*}}{\mathbf{B}} \overline{\bar{I}}_{\varepsilon^{*}, b}(j)\right) \sum_{k=1}^{2} \overline{\bar{I}}_{\varepsilon^{*}}^{k}(j)
$$

we have that its second moments are

$$
\frac{1}{\mathbf{B}^{2}}\left(\frac{\mathbf{b}_{2}^{*}-\mathbf{b}_{1}^{*}}{\mathbf{B}}\right)^{2}\left(\frac{\mathbf{j}_{2}^{*}-\mathbf{j}_{1}^{*}}{\tilde{n}}\right)^{2} \mathcal{E}^{*} I_{\varepsilon^{*}}^{2}(j)+o\left(T^{-1}\left(\frac{\mathbf{b}_{2}^{*}-\mathbf{b}_{1}^{*}}{\mathbf{B}}\right)^{2}\left(\frac{\mathbf{j}_{2}^{*}-\mathbf{j}_{1}^{*}}{\tilde{n}}\right)^{2}\right) H_{n}
$$


and hence, (5.27) is

$$
\frac{1}{\tilde{n} \mathbf{B}} \sum_{j=1}^{\mathbf{j}^{*}} \sum_{b=1}^{\mathbf{b}^{*}}\left\{{\stackrel{\circ}{\varepsilon^{*}, b}}(j)-\overline{\check{I}}_{\varepsilon^{*}}(j)\right\}+o\left(T^{-1}\left(\frac{\mathbf{b}_{2}^{*}-\mathbf{b}_{1}^{*}}{\mathbf{B}}\right)^{2}\left(\frac{\mathbf{j}_{2}^{*}-\mathbf{j}_{1}^{*}}{\tilde{n}}\right)^{2}\right) H_{n},
$$

which implies that

$$
[T / 2]^{1 / 2} \mathcal{T}_{n, \mathbf{B}}^{*}\left(\frac{\mathbf{b}^{*}}{\mathbf{B}} ; \frac{\mathbf{j}^{*}}{\tilde{n}}\right)=\frac{1}{[T / 2]^{1 / 2}} \sum_{j=1}^{\mathbf{j}^{*}} \sum_{b=1}^{\mathbf{b}^{*}}\left\{\stackrel{\circ}{I}_{\varepsilon^{*}, b}(j)-\overline{\stackrel{\circ}{I}}_{\varepsilon^{*}}(j)\right\}+o_{p^{*}}(1) .
$$

The proof is then completed if

$$
\breve{\mathcal{T}}_{n, \mathbf{B}}^{*}\left(\frac{\mathbf{b}^{*}}{\mathbf{B}} ; \frac{\mathbf{j}^{*}}{\tilde{n}}\right)=\frac{1}{\tilde{n}} \sum_{j=1}^{\mathbf{j}^{*}} \frac{1}{\mathbf{B}} \sum_{b=1}^{\mathbf{b}^{*}} \stackrel{\circ}{I}_{\varepsilon^{*}, b}(j) \stackrel{\text { weakly }}{\Longrightarrow} \mathcal{B W}\left([0,1]^{2}\right) \quad \text { (in probability). }
$$

Now, $\mathcal{E}^{*} \breve{\mathcal{T}}_{n, \mathbf{B}}^{*}\left(\frac{\mathbf{b}^{*}}{\mathbf{B}} ; \frac{\mathbf{j}^{*}}{\tilde{n}}\right)=0$, whereas by the independence of the sequence $\left\{{\stackrel{\circ}{\varepsilon^{*}, b}}(j)\right\}_{b=1}^{\mathbf{B}}$,

$$
\begin{aligned}
& {[T / 2] \mathcal{E}^{*}\left(\breve{\mathcal{T}}_{n, \mathbf{B}}^{*}\left(\frac{\mathbf{b}_{1}^{*}}{\mathbf{B}} ; \frac{\mathbf{j}_{1}^{*}}{\tilde{n}}\right) \breve{\mathcal{T}}_{n, \mathbf{B}}^{*}\left(\frac{\mathbf{b}_{2}^{*}}{\mathbf{B}} ; \frac{\mathbf{j}_{2}^{*}}{\tilde{n}}\right)\right) } \\
= & \frac{\left(\mathbf{b}_{1}^{*} \wedge \mathbf{b}_{2}^{*}\right)}{[T / 2]} \sum_{j=1}^{\mathbf{j}_{1}^{*}} \sum_{k=1}^{\mathbf{j}_{2}^{*}}\left(\mathcal{E}^{*}\left(\varepsilon_{t}^{* 2}\right) \mathcal{I}(j=k)+\frac{1}{n} \operatorname{Cum}^{*}\left(\varepsilon_{t}^{*} ; \varepsilon_{t}^{*} ; \varepsilon_{t}^{*} ; \varepsilon_{t}^{*}\right)\right) \\
& \stackrel{P}{\rightarrow}\left(\omega_{1}^{*} \wedge \omega_{2}^{*}\right)\left(v_{1}^{*}+\frac{1}{2} v_{1}^{*} v_{2}^{*} \kappa_{4}\right) .
\end{aligned}
$$

Finally, the tightness of $[T / 2]^{1 / 2} \breve{\mathcal{T}}_{n, \mathbf{B}}^{*}\left(\frac{\mathbf{b}^{*}}{\mathbf{B}} ; \frac{\mathbf{j}^{*}}{\tilde{n}}\right)$, for which a sufficient condition is that

$$
[T / 2]^{2} \mathcal{E}^{*}\left(\breve{\mathcal{T}}_{n, \mathbf{B}}^{* 4}\left(\frac{\left(\mathbf{j}_{2}^{*}-\mathbf{j}_{1}^{*}\right)}{\tilde{n}}, \frac{\left(\mathbf{b}_{2}^{*}-\mathbf{b}_{1}^{*}\right)}{\mathbf{B}}\right)\right)=\left(\omega_{1}^{*}-\omega_{2}^{*}\right)^{1+\delta}\left(v_{1}^{*}-v_{2}^{*}\right)^{1+\delta} H_{n}
$$

However, this proceeds by Lemma 7 in a standard way.

\section{APPENDIX III: AUXILIARY LEMMAS}

Before we present our lemmas, it is useful to introduce some notation. First from (2.10) and (2.11), we have that

$$
R_{n, b}(j)=\frac{\mathcal{B}(-j)}{|\mathcal{B}(-j)|^{2}} \mathcal{J}_{\varepsilon, b}(j) \mathrm{Y}_{n, b}(-j ; 0)+\frac{\mathcal{B}(j)}{|\mathcal{B}(j)|^{2}} \mathcal{J}_{\varepsilon, b}(-j) \mathrm{Y}_{n, b}(j ; 0)+\left|\mathrm{Y}_{n, b}(j ; 0)\right|^{2}
$$

where

$$
\mathrm{Y}_{n, b}(j ; 0)=\mathrm{Y}_{n, b}^{(1)}(j)+\mathrm{Y}_{n, b}^{(2)}(j)
$$

with $\mathcal{U}_{n \ell, b}(j)=\left\{\sum_{t=1-\ell}^{n-\ell}-\sum_{t=1}^{n}\right\} \varepsilon_{t+(b-1) n} e^{i t \lambda_{j}}$,

$$
\mathrm{Y}_{n, b}^{(1)}(j)=\frac{1}{n^{1 / 2}} \sum_{\ell=0}^{n} \beta(\ell) e^{-i \ell \lambda_{j}} \mathcal{U}_{n \ell, b}(j) ; \quad \mathrm{Y}_{n, b}^{(2)}(j)=\frac{1}{n^{1 / 2}} \sum_{\ell=n+1}^{\infty} \beta(\ell) e^{-i \ell \lambda_{j}} \mathcal{U}_{n \ell, b}(j)
$$

Further, we denote

$$
\mathrm{Z}_{n, b}^{(k)}(-j)=\mathcal{J}_{\varepsilon, b}(j) \mathrm{Y}_{n, b}^{(k)}(-j), \quad k=1,2
$$


Lemma 1. Assuming C1 and C2, we have that

$$
\begin{aligned}
\text { (a) }\left|\mathcal{E}\left(\prod_{q=1}^{4} \mathrm{Z}_{n, b}^{(1)}\left(j_{q}\right)\right)\right| & =\frac{d}{n^{2}}\left\{\frac{1}{n^{2}}+\mathcal{I}\left(j_{1}=j_{2}\right) \mathcal{I}\left(j_{3}=j_{4}\right)\right\} ; d<\infty \\
\text { (b) }\left|\mathcal{E}\left(\mathrm{Z}_{n, b}^{(1)}\left(j_{1}\right) \mathrm{Z}_{n, b}^{(1)}\left(j_{2}\right)\right)\right| & =\frac{d}{n}\left\{\frac{1}{n}+\mathcal{I}\left(j_{1}=j_{2}\right)\right\} \\
\text { (c) }\left|\mathcal{E}\left(\prod_{q=1}^{2 p} \mathrm{Z}_{n, b}^{(2)}\left(j_{q}\right)\right)\right| & =o\left(n^{-2 p}\right), \quad p=1,2 .
\end{aligned}
$$

Proof. We begin with part (c). We consider $p=2$, as the case for $p=1$ is addressed similarly. Because $\sum_{\ell=n+1}^{\infty}|\beta(\ell)|=o\left(n^{-1}\right)$ by $C 1$ and the definition of $\mathrm{Z}_{n, b}^{(2)}(-j)$, we have that the left side of the expression is bounded by

$$
\begin{aligned}
& \sum_{\ell_{1}, \ldots, \ell_{4}=n+1}^{\infty}\left|\prod_{q=1}^{4} \beta\left(\ell_{q}\right)\right|\left|\mathcal{E}\left(\prod_{q=1}^{4} \mathcal{J}_{\varepsilon, b}\left(j_{q}\right) \frac{1}{n^{1 / 2}} \mathcal{U}_{n \ell_{1}, b}\left(j_{q}\right)\right)\right| \\
= & o\left(n^{-4}\right) \mathcal{E}\left|\prod_{q=1}^{4} \mathcal{J}_{\varepsilon, b}\left(j_{q}\right) \frac{1}{n^{1 / 2}}\left\{\sum_{t=1-\ell}^{n-\ell}-\sum_{t=1}^{n}\right\} \varepsilon_{t+(b-1) n} e^{i t \lambda_{j_{q}}}\right| \\
= & o\left(n^{-4}\right),
\end{aligned}
$$

because $\sum_{\ell=0}^{n} \ell|\beta(\ell)|<\infty$ by $C 1$. The next part (a), which by definition is

$$
\begin{aligned}
& \frac{1}{n^{2}} \sum_{\ell_{1}, \ldots, \ell_{4}=0}^{n}\left|\prod_{q=1}^{4} \beta\left(\ell_{q}\right)\right|\left|E\left(\prod_{q=1}^{4} \mathcal{U}_{n \ell_{1}, b}\left(j_{q}\right) \mathcal{J}_{\varepsilon, b}\left(j_{q}\right)\right)\right| \\
= & \frac{d}{n^{2}} \sum_{\ell_{1}, \ldots, \ell_{4}=0}^{n}\left|\prod_{q=1}^{4} \beta\left(\ell_{q}\right) \ell_{q}\right|\left\{\frac{1}{n^{2}}+\left|E\left(\prod_{q=1}^{4} \mathcal{J}_{\varepsilon, b}\left(j_{q}\right)\right)\right|\right\},
\end{aligned}
$$

since $\mathcal{U}_{n \ell, b}(j)=\left\{\sum_{t=1-\ell}^{0}-\sum_{t=n-\ell+1}^{n}\right\} \varepsilon_{t+(b-1) n} e^{i t \lambda_{j}}$ when $\ell \leq n$, meaning that $\mathcal{E}\left(\mathcal{U}_{n \ell, b}\left(j_{q}\right) \mathcal{J}_{\varepsilon, b}\left(j_{q}\right)\right)=$ $O\left(\ell / n^{1 / 2}\right)$. Now, we conclude because $\sum_{\ell=0}^{n} \ell|\beta(\ell)|<\infty$. Finally, the proof of part (b) proceeds similarly.

Lemma 2. Assuming $C 1$ and $C 2$, we have that $q=1,2$,

$$
\begin{aligned}
& \mathcal{E}\left(\frac{1}{\mathbf{B}} \sum_{b=\mathbf{b}_{1}^{*}+1}^{\mathbf{b}_{2}^{*}} R_{n, b}(j)\right)^{2 q}=O\left(\left(\frac{\mathbf{b}_{2}^{*}-\mathbf{b}_{1}^{*}}{\mathbf{B}^{2} n}\right)^{q}+\left(\frac{\mathbf{b}_{2}^{*}-\mathbf{b}_{1}^{*}}{\mathbf{B} n}\right)^{2 q}\right) \\
& \mathcal{E} \sup _{j}\left|\frac{1}{\mathbf{B}} \sum_{b=\mathbf{b}_{1}^{*}+1}^{\mathbf{b}_{2}^{*}} R_{n, b}(j)\right|=O\left(\frac{\left(\mathbf{b}_{2}^{*}-\mathbf{b}_{1}^{*}\right)^{\frac{1}{2}}}{\mathbf{B}}+\frac{\mathbf{b}_{2}^{*}-\mathbf{b}_{1}^{*}}{\mathbf{B} n}\right)
\end{aligned}
$$

Proof. Because Brockwell and Davis's (1991) Theorem 10.3.1 and then $C 1$ and $H_{0}$ imply that $\mathcal{E}\left(R_{n, b}(j)\right)=$ $O\left(n^{-1}\right)$ and $\mathcal{E}\left|\mathrm{Y}_{n, b}(j ; 0)\right|^{4 q}=O\left(n^{-2 q}\right),(5.32)$ and (5.33) hold true if

$$
\begin{aligned}
& \mathcal{E}\left(\frac{1}{\mathbf{B}} \sum_{b=\mathbf{b}_{1}^{*}+1}^{\mathbf{b}_{2}^{*}}\left(\mathrm{Z}_{n, b}^{(k)}(-j)-\mathcal{E} \mathrm{Z}_{n, b}^{(k)}(-j)\right)\right)^{2 q}=O\left(\frac{\mathbf{b}_{2}^{*}-\mathbf{b}_{1}^{*}}{\mathbf{B}^{2} n}\right)^{q} k=1,2 ; \quad q=1,2 \\
& \sup _{1 \leq j \leq \tilde{n}}\left|\frac{1}{\mathbf{B}} \sum_{b=\mathbf{b}_{1}^{*}+1}^{\mathbf{b}_{2}^{*}}\left(\mathrm{Z}_{n, b}^{(k)}(-j)-\mathcal{E} \mathrm{Z}_{n, b}^{(k)}(-j)\right)\right|=O_{p}\left(\left(\frac{\mathbf{b}_{2}^{*}-\mathbf{b}_{1}^{*}}{\mathbf{B}^{2}}\right)^{1 / 2}\right), k=1,2 .
\end{aligned}
$$


Recall that $C^{-1}<|\mathcal{B}(\lambda)|^{2}<C$ for some positive finite constant $C$. However, (5.34) follows by Lemma 1 and $\mathrm{Z}_{n, b_{1}}^{(1)}(j)$ and $\mathrm{Z}_{n, b_{2}}^{(1)}(-k)$ are independent if $b_{1} \neq b_{2}$ by Condition $C 1$.

Next, we examine (5.35), which follows easily because its second moment is bounded by

$$
\sum_{j=1}^{\tilde{n}} \mathcal{E}\left|\frac{1}{\mathbf{B}} \sum_{b=\mathbf{b}_{1}^{*}+1}^{\mathbf{b}_{2}^{*}}\left(\mathrm{Z}_{n, b}^{(k)}(-j ; 0)-\mathcal{E} \mathrm{Z}_{n, b}^{(k)}(-j ; 0)\right)\right|^{2} \quad k=1,2 .
$$

This completes the proof of the lemma.

Lemma 3. Assuming $C 1$ and $C 2$, we have that for $q=1,2$,

$$
\mathcal{E}\left(\frac{1}{\tilde{n}} \sum_{j=\mathbf{j}_{1}^{*}+1}^{\mathbf{j}_{2}^{*}} \frac{1}{\mathbf{B}} \sum_{b=\mathbf{b}_{1}^{*}+1}^{\mathbf{b}_{2}^{*}} \breve{R}_{n, b}(j)\right)^{2 q}=O\left(\frac{1}{n^{2 q}}\left(\frac{\mathbf{j}_{2}^{*}-\mathbf{j}_{1}^{*}}{\tilde{n}}\right)^{1+\delta}\left(\frac{\mathbf{b}_{2}^{*}-\mathbf{b}_{1}^{*}}{\mathbf{B}}\right)^{1+\delta}\right) .
$$

Proof. We examine $q=1$, and the proof for $q=2$ proceeds similarly. By (5.29), (5.36) holds true if it also does for the second moments of

$$
\begin{aligned}
& \frac{1}{\tilde{n}} \sum_{j=\mathbf{j}_{1}^{*}+1}^{\mathbf{j}_{2}^{*}} \frac{1}{\mathbf{B}} \sum_{b=\mathbf{b}_{1}^{*}+1}^{\mathbf{b}_{2}^{*}}\left(\left|\mathrm{Y}_{n, b}(j ; 0)\right|^{2}-\mathcal{E}\left|\mathrm{Y}_{n, b}(j ; 0)\right|^{2}\right) \\
& \frac{1}{\tilde{n}} \sum_{j=\mathbf{j}_{1}^{*}+1}^{\mathbf{j}_{2}^{*}} \frac{1}{\mathbf{B}} \sum_{b=\mathbf{b}_{1}^{*}+1}^{\mathbf{b}_{2}^{*}}\left(\mathrm{Z}_{n, b}^{(k)}(-j ; 0)-\mathcal{E} \mathrm{Z}_{n, b}^{(k)}(-j ; 0)\right)
\end{aligned}
$$

for $k=1,2$. Following Brockwell and Davis's (1991) Theorem 10.3.2., the second moment of (5.37) satisfies the right side of (5.36) with $\delta=1$ there. Next, (5.38) when $k=2$. Because $\sum_{\ell=n}^{\infty}|\beta(\ell)|=o\left(n^{-1}\right)$ by Condition $C 1$ and that

$$
\frac{1}{(\tilde{n} \mathbf{B})^{1 / 2}} \sum_{j=\mathbf{j}_{1}^{*}+1}^{\mathbf{j}_{2}^{*}} \sum_{b=\mathbf{b}_{1}^{*}+1}^{\mathbf{b}_{2}^{*}}\left(\mathcal{J}_{\varepsilon, b}(j) \frac{1}{n^{1 / 2}} \sum_{t=1}^{n} \varepsilon_{t+(b-1) n} e^{-i t \lambda_{j}}-\mathcal{E}(\cdot)\right)
$$

converge to a Gaussian process, we have that (5.38) satisfies the right side of (5.36). Observe that the sequence is uniform integrable; by Serfling (1980), we have that the second moment of the sequence converges to that of the limiting distribution.

Finally, (5.37) when $k=1$. Because $\sum_{\ell=1}^{\infty} \ell|\beta(\ell)|<C$, it suffices to show that

$$
\mathcal{E}\left(\frac{1}{\tilde{n}^{3 / 2} \mathbf{B}} \sum_{j=\mathbf{j}_{1}^{*}+1}^{\mathbf{j}_{2}^{*}} \sum_{b=\mathbf{b}_{1}^{*}+1}^{\mathbf{b}_{2}^{*}}\left(\mathcal{J}_{\varepsilon, b}(j) \widetilde{\mathcal{J}}_{\varepsilon, b}(j, \ell)-\mathcal{E}\left(\mathcal{J}_{\varepsilon, b}(j) \widetilde{\mathcal{J}}_{\varepsilon, b}(j, \ell)\right)\right)\right)^{2},
$$

where $\widetilde{\mathcal{J}}_{\varepsilon, b}(j, \ell)=\ell^{-1} \sum_{t=n-\ell}^{n} \varepsilon_{t+(b-1) n} e^{-i t \lambda_{j}}$ satisfies the right side of (5.36). However, using Lemma 1 part (b), we have that it is

$$
\begin{aligned}
& O\left(\frac{\mathbf{j}_{2}^{*}-\mathbf{j}_{1}^{*}}{\tilde{n}^{3}} \frac{\mathbf{b}_{2}^{*}-\mathbf{b}_{1}^{*}}{\mathbf{B}^{2} \ell}\right)+O\left(\frac{\left(\mathbf{j}_{2}^{*}-\mathbf{j}_{1}^{*}\right)^{2}}{\tilde{n}^{4}} \frac{\mathbf{b}_{2}^{*}-\mathbf{b}_{1}^{*}}{\mathbf{B}^{2}}\right) \\
= & O\left(\frac{1}{n^{2}}\left(\frac{\mathbf{j}_{2}^{*}-\mathbf{j}_{1}^{*}}{\tilde{n}}\right)^{1+\delta}\left(\frac{\mathbf{b}_{2}^{*}-\mathbf{b}_{1}^{*}}{\mathbf{B}}\right)^{1+\delta}\right),
\end{aligned}
$$

for some $\delta>0$. 
Lemma 4. Assuming $C 1^{\prime}, C 2^{\prime}$ and $C 2$, we have that, $q=1,2$,

$$
\begin{aligned}
& \mathcal{E}\left(\frac{1}{\mathbf{B}} \sum_{b=\mathbf{b}_{1}^{*}+1}^{\mathbf{b}_{2}^{*}} R_{n, b}(j)\right)^{2 q}=O\left(\left(\frac{\mathbf{b}_{2}^{*}-\mathbf{b}_{1}^{*}}{\mathbf{B}^{2} n}\right)^{q}+\left(\frac{\mathbf{b}_{2}^{*}-\mathbf{b}_{1}^{*}}{\mathbf{B} n}\right)^{2 q}\right) \\
& \sup _{1 \leq j \leq \tilde{n}}\left|\frac{1}{\mathbf{B}} \sum_{b=\mathbf{b}_{1}^{*}+1}^{\mathbf{b}_{2}^{*}} R_{n, b}(j)\right|=O\left(\left(\frac{\mathbf{b}_{2}^{*}-\mathbf{b}_{1}^{*}}{\mathbf{B}^{2}}\right)^{1 / 2}+\frac{\mathbf{b}_{2}^{*}-\mathbf{b}_{1}^{*}}{\mathbf{B} n}\right)
\end{aligned}
$$

Proof. We examine $q=1$, as the proof for $q=2$ proceeds similarly. First, recall our decomposition in (2.27), that is,

$$
\mathcal{J}_{x, b}(j)=\mathcal{B}\left(\frac{n(b-1)}{T} ;-j\right) \mathcal{J}_{\varepsilon, b}(j)+\check{\mathrm{Y}}_{n, b}(j ; 0)+\ddot{\mathrm{Y}}_{n, b}(j) .
$$

Now, by definition, i.e., (2.29), and using (5.12) we have that

$$
\ddot{\mathrm{Y}}_{n, b+1}(j)=\frac{1}{n^{1 / 2}} \sum_{t=1}^{n} \sum_{\ell=0}^{\infty}\left(\dot{\beta}_{t+b n, T}(\ell)+\ddot{\beta}\left(\frac{t+n b}{T} ; \ell\right)\right) \varepsilon_{t+b n-\ell} e^{i t \lambda_{j}},
$$

and thus its contribution to (5.39) and (5.40) satisfies their right sides.

Proceeding as in the proof of Lemma 2 but with $f(j)$ replaced with $\left|\mathcal{B}\left(\frac{n(b-1)}{T} ; j\right)\right|^{2}$ and $\mathcal{B}(u ; j)$ given in (2.20), we have that the contribution due to the second term on the right side of (5.41) satisfies the statement of the lemma. Notice that there is no difference whether we have that the $M A$ representation of the process has weights $\beta(u ; \ell)$ or $\beta(\ell)$, as both sequences satisfy the same qualitative condition $\sum_{\ell=0}^{\infty} \ell|\beta(u ; \ell)|<\infty$.

Lemma 5. Assuming $C 1^{\prime}, C 2^{\prime}$ and $C 2$, we have that for $q=1,2$,

$$
\mathcal{E}\left(\frac{1}{\tilde{n}} \sum_{j=\mathbf{j}_{1}^{*}+1}^{\mathbf{j}_{2}^{*}} \frac{1}{\mathbf{B}} \sum_{b=\mathbf{b}_{1}^{*}+1}^{\mathbf{b}_{2}^{*}} \breve{R}_{n, b}(j)\right)^{2 q}=O\left(\frac{1}{n^{2 q}}\left(\frac{\mathbf{j}_{2}^{*}-\mathbf{j}_{1}^{*}}{\tilde{n}}\right)^{1+\delta}\left(\frac{\mathbf{b}_{2}^{*}-\mathbf{b}_{1}^{*}}{\mathbf{B}}\right)^{1+\delta}\right) .
$$

Proof. We examine $q=1$, as the proof for $q=2$ proceeds similarly. In view of (5.41) and the comments in Lemma 4, it suffices to show that

$$
\mathcal{E}\left(\frac{1}{\tilde{n}} \sum_{j=\mathbf{j}_{1}^{*}+1}^{\mathbf{j}_{2}^{*}} \frac{1}{\mathbf{B}} \sum_{b=\mathbf{b}_{1}^{*}+1}^{\mathbf{b}_{2}^{*}} \ddot{R}_{n, b}(j ; 0, \infty)\right)^{2}=O\left(\frac{1}{n^{2}}\left(\frac{\mathbf{j}_{2}^{*}-\mathbf{j}_{1}^{*}}{\tilde{n}}\right)^{1+\delta}\left(\frac{\mathbf{b}_{2}^{*}-\mathbf{b}_{1}^{*}}{\mathbf{B}}\right)^{1+\delta}\right),
$$

where

$$
\begin{aligned}
\ddot{R}_{n, b}\left(j ; q_{1}, q_{2}\right)= & \left|\frac{1}{n^{1 / 2}} \sum_{t=1}^{n}\left(\sum_{\ell=q_{1}}^{q_{2}} \dot{\beta}_{t, T}(\ell) \varepsilon_{t+(b-1) n-\ell}\right) e^{i t \lambda_{j}}\right|^{2} \\
& -\mathcal{E}\left|\frac{1}{n^{1 / 2}} \sum_{t=1}^{n}\left(\sum_{\ell=q_{1}}^{q_{2}} \dot{\beta}_{t, T}(\ell) \varepsilon_{t+(b-1) n-\ell}\right) e^{i t \lambda_{j}}\right|^{2} .
\end{aligned}
$$

By standard inequalities, the left side of (5.42) is bounded by

$$
\begin{aligned}
& \mathcal{E}\left(\frac{1}{\tilde{n}} \sum_{j=\mathbf{j}_{1}^{*}+1}^{\mathbf{j}_{2}^{*}} \frac{1}{\mathbf{B}} \sum_{b=\mathbf{b}_{1}^{*}+1}^{\mathbf{b}_{2}^{*}} \ddot{R}_{n, b}(j ; 0, n)\right)^{2}+\mathcal{E}\left(\frac{1}{\tilde{n}} \sum_{j=\mathbf{j}_{1}^{*}+1}^{\mathbf{j}_{2}^{*}} \frac{1}{\mathbf{B}} \sum_{b=\mathbf{b}_{1}^{*}+1}^{\mathbf{b}_{2}^{*}} \ddot{R}_{n, b}(j ; n, \infty)\right)^{2} . \\
\leq & \frac{\mathbf{j}_{2}^{*}-\mathbf{j}_{1}^{*}}{\tilde{n}^{2}} \sum_{j=\mathbf{j}_{1}^{*}+1}^{\mathbf{j}_{2}^{*}}\left\{\mathcal{E}\left(\frac{1}{\mathbf{B}} \sum_{b=\mathbf{b}_{1}^{*}+1}^{\mathbf{b}_{2}^{*}} \ddot{R}_{n, b}(j ; 0, n)\right)^{2}+\mathcal{E}\left(\frac{1}{\mathbf{B}} \sum_{b=\mathbf{b}_{1}^{*}+1}^{\mathbf{b}_{2}^{*}} \ddot{R}_{n, b}(j ; n, \infty)\right)^{2}\right\} .
\end{aligned}
$$


Now, the proof proceeds straightforwardly after noticing that if $b_{1} \neq b_{2}$, we have that $\mathcal{E}\left(\ddot{R}_{n, b_{1}}(j ; 0, n) \ddot{R}_{n, b_{2}}(j ; 0, n)\right)=0, \sum_{\ell>n} v(\ell)<C n^{-1}$ and $\left|\dot{\beta}_{t, T}(\ell)\right| \leq C v(\ell) / n^{-1 / 2}$ by (5.12). Details are omitted.

Let $|p|_{+}=\max \{1,|p|\}$ and denote

$$
\begin{aligned}
\psi_{k, n}(j) & =: \sum_{p=1}^{\tilde{n}} \varsigma_{p j} \overline{\bar{I}}_{\varepsilon}^{k}(p), \varphi_{k, n}(j)=: \sum_{p=1}^{\tilde{n}} \varsigma_{p j} \bar{R}_{n}^{k}(p) \quad k=1,2,3 \\
\Xi_{n}(j) & =\sum_{k=1}^{3} \frac{(-1)^{k}}{k !}\left(\psi_{k, n}(j)+\varphi_{k, n}(j)\right)+\Phi_{n, 1} \Phi_{n, 2}(j),
\end{aligned}
$$

where $\varsigma_{p j}=\left(|p-j|_{+}^{-1}+|p+j|^{-1}\right), \Phi_{n, 1}$ is a sequence of $O_{p}(1)$ r.v. independent of $\mathbf{j}^{*}$ and $\mathbf{b}^{*}$ and $\mathcal{E} \Phi_{n, 2}^{2}(j)=O\left(\mathbf{B}^{-2}\right)$. Moreover,

$$
\mathcal{A}_{n}(j)=: \exp \left\{\sum_{\ell=1}^{\tilde{n}} c_{\ell, n} e^{-i \ell \lambda_{j}}\right\} ; \quad c_{\ell, n}=\frac{1}{\tilde{n}} \sum_{p=1}^{\tilde{n}} \log f(p) \cos \left(\ell \lambda_{p}\right) .
$$

Lemma 6. Assuming $C 1$ and $C 2$, under $H_{0}$, we have that

$$
\begin{aligned}
& \text { (a) } \widehat{\mathcal{A}}(j)-\mathcal{A}_{n}(j)=\mathcal{A}(j) \Xi_{n}(j)+\frac{1}{2}|\mathcal{A}(j)|^{2}\left(\Xi_{n}(j)\right)^{2} \\
& \text { (b) } \mathcal{A}_{n}(j)-\mathcal{A}(j)=\mathcal{A}(j) \frac{\log f(0)}{\tilde{n}} \sum_{\ell=1}^{\tilde{n}} e^{-i \ell \lambda_{j}}+O\left(n^{-2}\right) .
\end{aligned}
$$

Proof. First because Taylor's expansion of $\log z$ yields that

$$
\begin{aligned}
\widehat{c}_{\ell}-c_{\ell, n}= & \frac{1}{\tilde{n}} \sum_{p=1}^{\tilde{n}} \sum_{k=1}^{3} \frac{(-1)^{k}}{k !}\left(\frac{\widehat{f}(p)-f(p)}{f(p)}\right)^{k} \cos \left(\ell \lambda_{p}\right) \\
& +\frac{1}{4 ! \tilde{n}} \sum_{p=1}^{\tilde{n}}\left(\frac{\widehat{f}(p)-f(p)}{\varkappa f(p)+(1-\varkappa) \hat{f}(p)}\right)^{4} \cos \left(\ell \lambda_{p}\right),
\end{aligned}
$$

where $\varkappa=: \varkappa(p) \in(0,1)$, meaning that

$$
\begin{aligned}
\log \left(\widehat{\mathcal{A}}(j) / \mathcal{A}_{n}(j)\right)= & \sum_{k=1}^{3} \frac{(-1)^{k}}{k !} \sum_{p=1}^{\tilde{n}} \varsigma_{p j}\left(\frac{\widehat{f}(p)-f(p)}{f(p)}\right)^{k} \\
& +\frac{1}{4 !} \sum_{p=1}^{\tilde{n}}\left(\frac{\widehat{f}(p)-f(p)}{\varkappa f(p)+(1-\varkappa) \widehat{f}(p)}\right)^{4}
\end{aligned}
$$

because $\sum_{\ell=1}^{\tilde{n}} \cos \left(\ell \lambda_{p}\right) e^{-i \ell \lambda_{j}}=\tilde{n} \varsigma_{p j}$. The second term on the left side of (5.46) is $\Phi_{n, 1} \Phi_{n, 2}(j)$, where $\Phi_{n, 1}=O_{p}(1)$ and $\mathcal{E}\left|\Phi_{n, 2}(j)\right|^{2}=O\left(\mathbf{B}^{-3}\right)$ uniformly in $j$. Indeed, Lemma 2 and $\sup _{p=1, \ldots, \tilde{n}}\left|a_{p}\right| \leq$ $\left(\sum_{p=1}^{\tilde{n}}\left|a_{p}\right|^{q}\right)^{1 / q}$ imply that

$$
\begin{aligned}
\mathcal{E} \sup _{p=1, \ldots, \tilde{n}}\left|\frac{\widehat{f}(p)-f(p)}{f(p)}\right| & \leq\left(\sum_{p=1}^{\tilde{n}} \mathcal{E}\left|\bar{R}_{n}(p)\right|^{4}\right)^{1 / 4}+\left(\sum_{p=1}^{\tilde{n}} \mathcal{E}\left(\overline{\tilde{I}}_{\varepsilon, b}(p)\right)^{4}\right)^{1 / 4} \\
& =O\left(\mathbf{B}^{-1 / 2} n^{1 / 4}\right)=o(1)
\end{aligned}
$$

by Condition $C 2$ and $\mathcal{E}\left(f^{-1}(p) \widehat{f}(p)-1\right)^{4}=O\left(\mathbf{B}^{-2}\right)$ by the standard arguments. Next, regarding the first term we have that because, say, $\mathcal{E}\left|\bar{I}_{\varepsilon}(p) \bar{R}_{n}(p)\right|^{2}=O\left(\mathbf{B}^{-2} T^{-1 / 2}\right)=o\left(\mathbf{B}^{-3}\right)$ by Lemma 2 and $C 2$ 
and that $\sup _{p}\left\{\left|\overline{\stackrel{I}{I}}_{\varepsilon}(p)\right|+\left|\bar{R}_{n}(p)\right|\right\}=o_{p}(1)$, we obtain that it is $\Xi_{n}(j)$. Now, we conclude the proof of part (a) by Taylor's expansion of $\exp z$.

Next, we address part (b). To that end, because $\log f(\lambda)$ is three times continuously differentiable, exercise 1.7.14, part $(b)$ in Brillinger (1981) implies that $c_{\ell, n}-c_{\ell}=\frac{\log f(0)}{\tilde{n}}+O\left(n^{-3}\right)$, and then we conclude that, uniformly in $j$,

$$
\begin{aligned}
\log \left(\mathcal{A}_{n}(j) / \mathcal{A}(j)\right) & =\sum_{\ell=1}^{\tilde{n}}\left(c_{\ell, n}-c_{\ell}\right) e^{-i \ell \lambda_{j}}-\sum_{\ell=\tilde{n}+1}^{\tilde{n}} c_{\ell} e^{-i \ell \lambda_{j}} \\
& =\frac{\log f(0)}{\tilde{n}} \sum_{\ell=1}^{\tilde{n}} e^{-i \ell \lambda_{j}}+O\left(n^{-2}\right) .
\end{aligned}
$$

Now, using (5.48) we obtain part (b). This concludes the proof.

Lemma 7. Assuming $C 1$ and $C 2$, we have that under $H_{0}$ for all $b=1, \ldots, \mathbf{B}$,

$$
\widehat{\sigma}_{\varepsilon}^{2}(b)-\widetilde{\sigma}_{\varepsilon}^{2}(b)=\frac{d_{1, n}}{n} \sum_{j=1}^{n} \mathcal{A}^{-1}(j) \psi_{1, n}(j) \stackrel{\circ}{I}_{\varepsilon, b}(j)+\frac{d_{2, n}}{\mathbf{B}}+\Psi_{n, 1} \Psi_{n, 2}(b),
$$

where $\Psi_{n, 1} d_{1, n}$ and $d_{2, n}$ are independent of $b$ such that $\Psi_{n, 1}=O_{p}(1), \mathcal{E}\left(d_{2, n}^{2}\right)<C$ and $\mathcal{E}\left|\Psi_{n, 2}(b)\right|^{2}=$ $O\left(\mathbf{B}^{-3}+n^{-2}\right)$ with $\tilde{\sigma}_{\varepsilon}^{2}(b)$ given in $(5.4)$.

Proof. First, by standard algebra, we have that

$$
\widehat{\sigma}_{\varepsilon}^{2}(b)-\widetilde{\sigma}_{\varepsilon}^{2}(b)=\frac{1}{n} \sum_{t=1}^{n} v_{t, b}^{2}+\frac{2}{n} \sum_{t=1}^{n} \varepsilon_{t+(b-1) n} v_{t, b},
$$

where $v_{t, b}=: \widehat{\varepsilon}_{t+(b-1) n}-\varepsilon_{t+(b-1) n}$, and it is

$$
\begin{aligned}
v_{t, b}= & \frac{1}{n^{1 / 2}} \sum_{j=1}^{n} e^{i t \lambda_{j}}\left(\widehat{\mathcal{A}}(j) \mathcal{A}^{-1}(j)-1\right) \mathcal{A}(j) \mathrm{Y}_{n, b}(j ; 0) \\
& +\frac{1}{n^{1 / 2}} \sum_{j=1}^{n} e^{i t \lambda_{j}}\left(\widehat{\mathcal{A}}(j) \mathcal{A}^{-1}(j)-1\right) \mathcal{J}_{\varepsilon, b}(j) \\
& +\frac{1}{n} \sum_{j=1}^{n} e^{i t \lambda_{j}} \mathcal{A}(j) \sum_{s=1}^{n} x_{s+(b-1) n} e^{-i s \lambda_{j}}-\varepsilon_{t+(b-1) n} .
\end{aligned}
$$

Using (2.10) and because $\mathcal{A}(j)=\sum_{q=0}^{\infty} \alpha(q) e^{-i q \lambda_{j}}$ and $\sum_{j=1}^{n} e^{-i \ell \lambda_{j}}=n \mathcal{I}(\ell=0, n, \ldots)$, we obtain that the third term of $(5.51)$, with $b=1$ for notational simplicity, is

$$
\begin{aligned}
& \sum_{q=0}^{\infty} \alpha(q) \sum_{s=1}^{n} x_{s} \frac{1}{n} \sum_{j=1}^{n} e^{i(t-q-s) \lambda_{j}}-\varepsilon_{t} \\
= & \sum_{\ell=1}^{\infty} \sum_{q=1}^{t-1} \alpha(q+\ell n) x_{t-q}+\left\{\sum_{q=1}^{t-1} \alpha(q) x_{t-q}-\varepsilon_{t}\right\},
\end{aligned}
$$

the second moment of which is $o\left((t \log (t+1))^{-2}\right)$. Thus, the contribution due to the third term of (5.51) into (5.50) is such that its second moment is $O\left(n^{-2}\right)$. 
Now, the contribution due to the first two terms on the right side of $(5.51)$ to $\widehat{\sigma}_{\varepsilon}^{2}(b)-\widetilde{\sigma}_{\varepsilon}^{2}(b)$ is

$$
\begin{aligned}
& \frac{1}{n} \sum_{j=1}^{n}(\widehat{\mathcal{A}}(j)-\mathcal{A}(j))^{2}\left|\mathrm{Y}_{n, b}(j ; 0)\right|^{2} \\
& +\frac{2}{n} \sum_{j=1}^{n}(\widehat{\mathcal{A}}(j)-\mathcal{A}(j))\left(\mathcal{A}^{-1}(j)+\frac{1}{2}\right) \mathcal{J}_{\varepsilon, b}(-j) \mathrm{Y}_{n, b}(j ; 0) \\
& +\frac{1}{n} \sum_{j=1}^{n}\left\{\frac{(\widehat{\mathcal{A}}(j)-\mathcal{A}(j))^{2}}{|\mathcal{A}(j)|^{2}}+(\widehat{\mathcal{A}}(j)-\mathcal{A}(j)) \mathcal{A}^{-1}(j)\right\} I_{\varepsilon, b}(j) .
\end{aligned}
$$

The contribution due to $\Phi_{n, 1} \Phi_{n, 2}(j)$ to the first term of (5.53) is

$$
\Phi_{n, 1}^{2} \frac{1}{n} \sum_{j=1}^{n} \Phi_{n, 2}^{2}(j)\left|\mathrm{Y}_{n, b}(j ; 0)\right|^{2} .
$$

Now, we identify $\Phi_{n, 1}^{2} \sup _{j} \Phi_{n, 2}(j)$ with $\Psi_{n, 1}$ and

$$
\Psi_{n, 2}(b)=: \frac{1}{n} \sum_{j=1}^{n}\left|\Phi_{n, 2}(j)\right|\left|\mathrm{Y}_{n, b}(j ; 0)\right|^{2}
$$

noticing that $\mathcal{E} \Psi_{n, 2}^{2}(b)=O\left(T^{-2}\right)=o\left(n^{-2}+\mathbf{B}^{-3}\right)$ because $\mathcal{E}\left|\mathrm{Y}_{n, b}(j ; 0)\right|^{2 p}=O\left(n^{-p}\right)$ and CauchySchwarz's inequality. Next, we address the contribution due to $\psi_{k, n}(j)+\varphi_{k, n}(j)$, for $k=1,2,3$. Now, since $(a+b)^{4} \leq 8\left(a^{4}+b^{4}\right)$ and $\mathcal{E}\left|\psi_{k, n}(j)\right|^{2}+\mathcal{E}\left|\varphi_{k, n}(j)\right|^{2}=O\left(\mathbf{B}^{-k}\right)$, we have that this contribution is also $\Psi_{n, 1} \Psi_{n, 2}(b)$. Recall again that $\sup _{j}\left|\psi_{k, n}(j)\right|=o_{p}(1)$ and $\sup _{j}\left|\varphi_{k, n}(j)\right|=o_{p}(1)$.

Next we examine the behavior of the second term of (5.53). To that end and using (5.31), we first notice that Condition $C 1$ implies that $\mathcal{E}\left|\mathrm{Z}_{n, b}^{(k)}(-j)\right|^{2}=O\left(n^{-1}\right)$, for $k=1,2$, and hence that

$$
\mathcal{E}\left|\frac{\log f(0)}{\tilde{n}} \frac{1}{n} \sum_{j=1}^{n} \mathrm{Z}_{n, b}^{(k)}(-j) \sum_{\ell=1}^{\tilde{n}} e^{-i \ell \lambda_{j}}\right|^{2}=O\left(\frac{\log ^{2} n}{n^{3}}\right)
$$

by standard arguments. Next, because $\sup _{j}\left|\psi_{k, n}(j)\right|=o_{p}(1)$ and $\sup _{j}\left|\varphi_{k, n}(j)\right|=o_{p}(1)$, we have that $\psi_{k, n}^{4}(j)=\xi_{n, 1} \xi_{n, 2}$, where $\xi_{n, 1}=O_{p}(1)$ and $\mathcal{E}\left(\xi_{n, 2}\right)^{2}=O\left(\mathbf{B}^{-3}\right)$, we then have that the second term of (5.53), except the multiplicative constants, is

$$
\begin{aligned}
& \frac{1}{n} \sum_{j=1}^{n} \psi_{1, n}(j)\left(\mathrm{Z}_{n, b}^{(1)}(-j)+\mathrm{Z}_{n, b}^{(2)}(-j)\right)+\Psi_{n, 1} \Psi_{n, 2}(b) \\
= & \frac{1}{n} \sum_{j=1}^{n} \psi_{1, n}(j) \mathrm{Z}_{n, b}^{(1)}(-j)+\Psi_{n, 1} \Psi_{n, 2}(b)
\end{aligned}
$$

as $\mathcal{E}\left(\varphi_{k, n}^{2}(j)\right)=O\left(T^{-k}\right)$. Now, proceeding as with the proof of Lemma 2 and using the definition of $\psi_{1, n}(j)$, it suffices to examine the behavior of

$$
\begin{aligned}
& \frac{1}{n^{1 / 2} \mathbf{B}} \frac{1}{n} \sum_{j=1}^{n} \stackrel{\circ}{I}_{\varepsilon, b}(j) \mathcal{J}_{\varepsilon, b}(-j) \frac{1}{\ell^{1 / 2}} \sum_{t=n-\ell+1}^{n} \varepsilon_{t+(b-1) n} e^{i t \lambda_{j}} \\
& +\frac{1}{n^{1 / 2}} \frac{1}{n} \sum_{j=1}^{n}\left(\frac{1}{\mathbf{B}} \sum_{b_{1} \neq b}^{\mathbf{B}} \stackrel{\circ}{I}_{\varepsilon, b_{1}}(j)\right)\left(\mathcal{J}_{\varepsilon, b}(-j) \frac{1}{\ell^{1 / 2}} \sum_{t=n-\ell+1}^{n} \varepsilon_{t+(b-1) n} e^{i t \lambda_{j}}\right) .
\end{aligned}
$$


The second moment of the first term of the last displayed expression is clearly $O\left(n^{-1} \mathbf{B}^{-2}\right)$, whereas the second term is $O\left(n^{-2} \mathbf{B}^{-1}\right)$ because the first factor in parentheses is independent of the second factor and

$$
\mathcal{E}\left(\stackrel{\circ}{I}_{\varepsilon, b_{1}}(j) \stackrel{\circ}{I}_{\varepsilon, b_{2}}(k)\right)=\mathcal{I}\left(b_{1}=b_{2}\right)\left(\mathcal{I}(j=k)+n^{-1} \kappa_{4}\right) .
$$

To complete the proof of the lemma, we now examine the third term of (5.53). First, using (5.43) and that $\mathcal{E}\left|\psi_{k, n}(j)\right|^{2 p}+\mathcal{E}\left|\varphi_{k, n}(j)\right|^{2 p}=O\left(\mathbf{B}^{-3}\right)$ when $p+k \geq 3$, the third term of (5.53) is

$$
\frac{1}{n} \sum_{j=1}^{n}\left\{\sum_{k=1}^{2} \frac{d_{k, n}}{\mathcal{A}(j)}\left(\psi_{k, n}(j)+\varphi_{k, n}(j)\right)+d\left(\psi_{1, n}^{2}(j)+\varphi_{1, n}^{2}(j)\right)\right\} I_{\varepsilon, b}(j)+\Psi_{n},
$$

$d_{1, n}$ and $d_{2, n}$ independent of $b$ and finite second moments and $d \geq|\mathcal{A}(j)|^{-2}$ finite. Now, because $\mathcal{E}\left|\mathbf{B} \psi_{2, n}(j)\right|=O(1)$, we have that

$$
\begin{aligned}
\frac{1}{n} \sum_{j=1}^{n} \frac{1}{\mathcal{A}(j)} \psi_{2, n}(j) I_{\varepsilon, b}(j) & =\frac{d_{2, n}}{\mathbf{B}}+\frac{1}{n} \sum_{j=1}^{n} \mathcal{A}^{-1}(j) \psi_{2, n}(j) \stackrel{\circ}{I}_{\varepsilon, b}(j) \\
& =\frac{d_{2, n}}{\mathbf{B}}+\Psi_{n},
\end{aligned}
$$

as we now show. Indeed, the second term on the right side of (5.57) is

$$
\begin{aligned}
& \frac{1}{\mathbf{B}^{2} n} \sum_{j=1}^{n} \mathcal{A}^{-1}(j) \sum_{p=1}^{\tilde{n}} \varsigma_{p j} \stackrel{\circ}{I}_{\varepsilon, b}^{2}(p) \stackrel{\circ}{I}_{\varepsilon, b}(j) \\
& +\frac{1}{n} \sum_{j=1}^{n} \mathcal{A}^{-1}(j) \sum_{p=1}^{\tilde{n}} \varsigma_{p j}\left(\frac{1}{\mathbf{B}} \sum_{b_{1} \neq b}^{\mathbf{B}} \stackrel{\circ}{I}_{\varepsilon, b_{1}}(p)\right)^{2} \stackrel{\circ}{I}_{\varepsilon, b}(j) \\
& +\frac{2}{\mathbf{B} n} \sum_{j=1}^{n} \mathcal{A}^{-1}(j) \sum_{p=1}^{\tilde{n}} \varsigma_{p j}\left(\frac{1}{\mathbf{B}} \sum_{b_{1} \neq b}^{\mathbf{B}} \stackrel{\circ}{I}_{\varepsilon, b_{1}}(p)\right) \stackrel{\circ}{I}_{\varepsilon, b}(p) \stackrel{\circ}{I}_{\varepsilon, b}(j) .
\end{aligned}
$$

The second moment of the first term of (5.58) is $O\left(\mathbf{B}^{-4}\right)$, whereas the second moment of the third term is $O\left(\mathbf{B}^{-3}\right)$, as $\mathcal{E}\left(\stackrel{\circ}{I}_{\varepsilon, b_{1}}(p) \stackrel{\circ}{I}_{\varepsilon, b}(j)\right)=0$ for all $b_{1} \neq b$. Thus, we are left to examine the second term of (5.58). However, its second moment is clearly $O\left(\mathbf{B}^{-3}\right)$, as by the independence of $\stackrel{\circ}{I}_{\varepsilon, b}(j)$ and $\left(\frac{1}{\mathbf{B}} \sum_{b_{1} \neq b}^{\mathbf{B}} \stackrel{\circ}{I}_{\varepsilon, b_{1}}(p)\right)^{2}$ and $(5.55)$, we have that the second moment is

$$
\frac{d}{\mathbf{B}^{2} n^{2}} \sum_{j=1}^{n} \sum_{p=1}^{\tilde{n}} \varsigma_{p j}^{2}|\mathcal{A}(j)|^{-2}=o\left(\mathbf{B}^{-3}\right)
$$

by Condition $C 2$.

Next, we examine the contribution to (5.56), i.e., the third term of (5.53), due to

$$
\begin{aligned}
\frac{1}{n} \sum_{j=1}^{n} \mathcal{A}^{-1}(j) \varphi_{2, n}(j) I_{\varepsilon, b}(j) & =\frac{d_{2, n}}{\mathbf{B}}+\frac{1}{n} \sum_{j=1}^{n} \mathcal{A}^{-1}(j) \varphi_{2, n}(j) \stackrel{\circ}{I}_{\varepsilon, b}(j) \\
& =\frac{d_{2, n}}{\mathbf{B}}+\Psi_{n},
\end{aligned}
$$


because $\mathcal{E} \varphi_{2, n}^{2}(j)=O\left(T^{-1}\right)=o\left(\mathbf{B}^{-2}\right)$ by Condition $C 2$. Regarding $\Psi_{n}$, by definition of $\varphi_{2, n}(j)$, we need to examine

$$
\begin{aligned}
& \sum_{\ell=1}^{2} \frac{1}{n} \sum_{j=1}^{n} \mathcal{A}^{-1}(j) \stackrel{\circ}{I}_{\varepsilon, b}(j) \sum_{p=1}^{\tilde{n}} \varsigma_{p j}\left(\frac{1}{\mathbf{B}} \sum_{b=1}^{\mathbf{B}} \mathrm{Z}_{n, b}^{(\ell)}(-p)\right)^{2} \\
& +\frac{1}{n} \sum_{j=1}^{n} \mathcal{A}^{-1}(j) \stackrel{\circ}{I_{\varepsilon, b}}(j) \sum_{p=1}^{\tilde{n}} \varsigma_{p j}\left(\frac{1}{\mathbf{B}} \sum_{b=1}^{\mathbf{B}}\left|\mathrm{Y}_{n, b}(p ; 0)\right|^{2}\right)^{2} .
\end{aligned}
$$

However, it is clear that the second moment is $O\left(\mathbf{B}^{-3}+n^{-2}\right)$ because $\mathcal{E}\left|\mathrm{Y}_{n, b}(j ; 0)\right|^{4}=O\left(n^{-2}\right)$,

$$
\mathcal{E}\left(\frac{1}{\mathbf{B}} \sum_{b=1}^{\mathbf{B}} \mathrm{Z}_{n, b}^{(2)}(-j)\right)^{4}=O\left(n^{-4}\right) ; \quad \mathcal{E}\left(\frac{1}{\mathbf{B}} \sum_{b=1}^{\mathbf{B}} \mathrm{Z}_{n, b}^{(1)}(-j)\right)^{4}=O\left(n^{-2}\right)
$$

by simple inspection of the definition of $\mathrm{Y}_{n, b}(j ; 0), \mathrm{Z}_{n, b}^{(1)}(j)$ and $\mathrm{Z}_{n, b}^{(2)}(j)$, respectively.

Next, we examine the contribution to (5.56), i.e., the third term of (5.53), due to

$$
\begin{aligned}
& \frac{1}{n} \sum_{j=1}^{n} \frac{\psi_{1, n}^{2}(j)+\varphi_{1, n}^{2}(j)}{|\mathcal{A}(j)|^{2}} \stackrel{\circ}{\varepsilon}, b(j)=\frac{1}{n} \sum_{j=1}^{n} \frac{\stackrel{\circ}{I}_{\varepsilon, b}(j)}{|\mathcal{A}(j)|^{2}}\left(\sum_{p=1}^{\tilde{n}} \varsigma_{p j} \overline{\bar{I}}_{\varepsilon}(p)\right)^{2} \\
& +\frac{1}{n} \sum_{j=1}^{n} \frac{\stackrel{\circ}{I}_{\varepsilon, b}(j)}{|\mathcal{A}(j)|^{2}}\left(\sum_{p=1}^{\tilde{n}} \varsigma_{p j} \bar{R}_{n}(p)\right)^{2} .
\end{aligned}
$$

The first term on the right side of (5.60) is

$$
\begin{aligned}
& \frac{1}{n} \sum_{j=1}^{n} \frac{\stackrel{\circ}{I}_{\varepsilon, b}(j)}{|\mathcal{A}(j)|^{2}}\left(\sum_{p=1}^{\tilde{n}} \varsigma_{p j} \frac{1}{\mathbf{B}} \sum_{b_{1} \neq b}^{\mathbf{B}} \stackrel{\circ}{I}_{\varepsilon, b_{1}}(p)\right)^{2} \\
& +\frac{1}{\mathbf{B}^{2} n} \sum_{j=1}^{n} \frac{\stackrel{\circ}{I}_{\varepsilon, b}(j)}{|\mathcal{A}(j)|^{2}}\left(\sum_{p=1}^{\tilde{n}} \varsigma_{p j} \stackrel{\circ}{I}_{\varepsilon, b}(p)\right)^{2} \\
& +\frac{2}{\mathbf{B} n} \sum_{j=1}^{n} \frac{\stackrel{\circ}{I}_{\varepsilon, b}(j)}{|\mathcal{A}(j)|^{2}} \sum_{p_{1}, p_{2}=1}^{\tilde{n}} \varsigma_{p_{1} j} \varsigma_{p_{2} j} \stackrel{\circ}{I}_{\varepsilon, b}\left(p_{2}\right)\left(\frac{1}{\mathbf{B}} \sum_{b_{1} \neq b}^{\mathbf{B}} \stackrel{\circ}{I}_{\varepsilon, b_{1}}\left(p_{1}\right)\right) .
\end{aligned}
$$

Clearly, the second moments of the second and third terms are $O\left(\mathbf{B}^{-4}+\mathbf{B}^{-3}\right)$, whereas the second moment of the first term is, by the independence of ${\stackrel{\circ}{\varepsilon}, b_{1}}_{1}(p)$ and $\stackrel{\circ}{I}, b_{\varepsilon}(p)$ if $b_{1} \neq b$, and (5.55) is easy to observe that is $O\left(\mathbf{B}^{-2} n^{-1}\right)$. Next, the second term on the right side of (5.60) also satisfies that its second moment is $O\left(\mathbf{B}^{-3}+n^{-2}\right)$ using Lemma 3. To complete the proof, it remains to examine the behavior of

$$
\begin{aligned}
& \frac{1}{n} \sum_{j=1}^{n} \varphi_{1, n}(j) \frac{\stackrel{\circ}{I}_{\varepsilon, b}(j)}{\mathcal{A}(j)} \\
& \frac{1}{n} \sum_{j=1}^{n} \mathcal{A}^{-1}(j)\left(\psi_{1, n}(j)+\varphi_{1, n}(j)\right) .
\end{aligned}
$$


Now, (5.61) is

$$
\begin{aligned}
& \frac{1}{n} \sum_{p=1}^{\tilde{n}}\left(\frac{1}{\mathbf{B}} \sum_{b=1}^{\mathbf{B}} \mathrm{Z}_{n, b}^{(1)}(-j)\right) \sum_{j=1}^{n} \mathcal{A}^{-1}(j) \varsigma_{p j} \stackrel{\circ}{I}_{\varepsilon, b}(j) \\
& +\frac{1}{n} \sum_{p=1}^{\tilde{n}}\left(\frac{1}{\mathbf{B}} \sum_{b=1}^{\mathbf{B}} \mathrm{Z}_{n, b}^{(2)}(-j)\right) \sum_{j=1}^{n} \mathcal{A}^{-1}(j) \varsigma_{p j} \stackrel{\circ}{I}_{\varepsilon, b}(j) \\
& +\frac{1}{n} \sum_{p=1}^{\tilde{n}}\left(\frac{1}{\mathbf{B}} \sum_{b=1}^{\mathbf{B}}\left|\mathrm{Y}_{n, b}(j ; 0)\right|^{2}\right) \sum_{j=1}^{n} \mathcal{A}^{-1}(j) \varsigma_{p j} \stackrel{\circ}{I}_{\varepsilon, b}(j) .
\end{aligned}
$$

The second and third terms of the last displayed expression have second moments $O\left(n^{-2}\right)$, whereas the first term proceeding similarly to (5.54) has a second moment $O\left(\mathbf{B}^{-3}+n^{-2}\right)$. Finally, we have (5.62), which is

$$
\frac{1}{n} \sum_{p=1}^{\tilde{n}}\left(\frac{1}{\mathbf{B}} \sum_{b=1}^{\mathbf{B}} \stackrel{\circ}{I}_{\varepsilon, b}(p)\right)+\left(\frac{1}{\mathbf{B}} \sum_{b=1}^{\mathbf{B}} R_{n, b}(p)\right) \sum_{j=1}^{n} \varsigma_{p j} \mathcal{A}^{-1}(j) .
$$

By (5.55), the first term has second moment proportional to

$$
\frac{1}{\mathbf{B} n^{2}} \sum_{p=1}^{\tilde{n}} \sum_{j_{1}, j_{2}=1}^{n}\left|\varsigma_{p j_{1}} \varsigma_{p j_{2}}\right|=\mathcal{O}\left(\frac{\log ^{2} n}{T}\right)=O\left(\mathbf{B}^{-2}\right),
$$

whereas by Lemma 2 and Condition $C 2$, the second term is also $O\left(\mathbf{B}^{-2}\right)$. This concludes the proof of the lemma.

Lemma 8. Assuming $C 1^{\prime}, C 2^{\prime}$ and $C 2$, we have that for all $b=1, \ldots, \mathbf{B}$ and uniformly in $t, v_{t, b}=$ $O_{p}\left((t \log (t+1))^{-1}+n^{1 / 2} \mathbf{B}^{-1}\right)$.

Proof. By (5.51) and (5.52), it suffices to examine

$$
\begin{aligned}
& \frac{1}{n^{1 / 2}} \sum_{j=1}^{n} e^{i t \lambda_{j}}\left(\widehat{\mathcal{A}}(j) \mathcal{A}^{-1}(j)-1\right) \mathcal{A}(j) \mathrm{Y}_{n, b}(j ; 0) \\
& +\frac{1}{n^{1 / 2}} \sum_{j=1}^{n} e^{i t \lambda_{j}}\left(\widehat{\mathcal{A}}(j) \mathcal{A}^{-1}(j)-1\right) \mathcal{J}_{\varepsilon, b}(j) .
\end{aligned}
$$

(5.43) and (5.44) imply that the first term of the last displayed expression is $O_{p}\left(\mathbf{B}^{-1 / 2}\right)$ uniformly in $t$ as $\mathcal{E}\left(f^{-1}(\ell) \widehat{f}(\ell)-1\right)^{2}=O\left(\mathbf{B}^{-1}\right)$ and Cauchy-Schwarz's inequality, whereas the second term is

$$
\begin{aligned}
& \frac{1}{n^{1 / 2}} \sum_{j=1}^{n} e^{i t \lambda_{j}} \psi_{1, n}(j) \mathcal{J}_{\varepsilon, b}(j)+O_{p}\left(n^{1 / 2} \mathbf{B}^{-1}\right) \\
= & \frac{1}{n^{1 / 2}} \sum_{j=1}^{n} e^{i t \lambda_{j}} \overline{\bar{I}}_{\varepsilon}(j) \mathcal{J}_{\varepsilon, b}(j)+O_{p}\left(n^{1 / 2} \mathbf{B}^{-1}+\mathbf{B}^{-1 / 2}\right)
\end{aligned}
$$

again uniformly in $t$, proceeding with arguments in Lemma 7 and Lemma 2. Now, the first term on the right is

$$
\frac{1}{n^{1 / 2}} \sum_{j=1}^{n} e^{i t \lambda_{j}}\left(\frac{1}{\mathbf{B}} \sum_{b_{1} \neq b}^{\mathbf{B}} \stackrel{\circ}{I}_{\varepsilon, b_{1}}(j)\right) \mathcal{J}_{\varepsilon, b}(j)+\frac{1}{\mathbf{B}} \frac{1}{n^{1 / 2}} \sum_{j=1}^{n} e^{i t \lambda_{j}} \stackrel{\circ}{I}_{\varepsilon, b}(j) \mathcal{J}_{\varepsilon, b}(j) .
$$

However, it is easy to see that the fourth moment of the first term is $O\left(\mathbf{B}^{-2}\right)$, whereas the second term has a second moment of order $O\left(\mathbf{B}^{-2}\right)$. Thus, using that $\sup _{\ell=1, \ldots, \tilde{n}}\left|a_{\ell}\right|^{q} \leq \sum_{\ell}\left|a_{\ell}\right|^{q}$, for $q \geq 1$, we conclude that the last displayed expression is $O\left(n \mathbf{B}^{-2}\right)$ uniformly in $t$. 
Lemma 9. Assuming $C 1^{\prime}$ and C2, we have that

$$
\mathcal{E}^{*}\left(\sum_{b=\mathbf{b}_{1}^{*}+1}^{\mathbf{b}_{2}^{*}}{\stackrel{\circ}{I^{*}, b}}_{\varepsilon^{2}}(j)\right)^{2 \ell}=\left(\mathbf{b}_{2}^{*}-\mathbf{b}_{1}^{*}\right)^{\ell} H_{n}, \quad \ell \geq 1,
$$

$\left\{H_{n}\right\}_{n \geq 1}$ being a sequence of strictly positive $O_{p}(1)$ random variables

Proof. Because $\stackrel{\circ}{I}_{\varepsilon^{*}, b_{1}}(j)$ and $\stackrel{\circ}{I}_{\varepsilon^{*}, b_{2}}(j)$ are independent for $b_{1} \neq b_{2}$, we have that the left side of (5.63) is bounded by

$$
\begin{aligned}
& \sum_{b=\mathbf{b}_{1}^{*}+1}^{\mathbf{b}_{2}^{*}} \mathcal{E}^{*}\left(\stackrel{\circ}{I}_{\mathcal{E}^{*}, b}(j)\right)^{2 \ell}+\left(\begin{array}{c}
2 \ell \\
2
\end{array}\right) \sum_{b_{1} \neq b_{2}=\mathbf{b}_{1}^{*}+1}^{\mathbf{b}_{2}^{*}} \mathcal{E}^{*}\left(\stackrel{\circ}{I}_{\mathcal{E}^{*}, b_{1}}(j)\right)^{2} \mathcal{E}^{*}\left(\stackrel{\circ}{I}_{\varepsilon^{*}, b_{2}}(j)\right)^{2 \ell-2} \\
& \left.+\ldots+\left(\begin{array}{c}
2 \ell \\
\ell
\end{array}\right)_{b_{1} \neq \ldots \neq b_{\ell}=\mathbf{b}_{1}^{*}+1} \sum_{p=1}^{\ell}\left(\prod_{\mathbf{b}^{*}}^{\ell} \mathcal{E}_{\dot{\varepsilon}^{*}, b_{p}}(j)\right)^{2}\right) .
\end{aligned}
$$

However, $\mathcal{E}^{*}\left(\stackrel{\circ}{I}_{\varepsilon^{*}, b}(j)\right)^{2 \chi}=H_{n}$ because for all integers $\chi \geq 1$,

$$
\mathcal{E}^{*}\left(\frac{1}{n} \sum_{t=1}^{n} \varepsilon_{t+(n-1) b}^{* \chi}\right)=\frac{1}{T} \sum_{t=1}^{n} \widehat{\varepsilon}_{t}^{\chi}
$$

and by Theorem 1 and then $C 1^{\prime}$, we have that

$$
\frac{1}{T} \sum_{t=1}^{n}\left(\hat{\varepsilon}_{t}^{\chi}-\varepsilon_{t}^{\chi}\right)=o_{p}(1) ; \quad \frac{1}{T} \sum_{t=1}^{n}\left(\varepsilon_{t}^{\chi}-\mathcal{E} \varepsilon_{t}^{\chi}\right)=O_{p}\left(\frac{1}{T^{1 / 2}}\right) .
$$

This completes the proof of the lemma.

Economics Department, London School of Economics, Houghton Street, London WC2A 2AE, U.K.

E-mail address: f.j.hidalgo@lse.ac.uk

Current address: Economics Department, University of Warwick, Coventry CV4 7AL, U.K.

$U R L:$ http://www.pedroclsouza.com 